\title{
Search for flavor-changing neutral currents in top quark decays $\boldsymbol{t} \rightarrow \boldsymbol{H c}$ and $t \rightarrow H u$ in multilepton final states in proton-proton collisions at $\sqrt{s}=13 \mathrm{TeV}$ with the ATLAS detector
}

\author{
M. Aaboud et al." \\ (ATLAS Collaboration)
}

(Received 10 May 2018; published 6 August 2018)

\begin{abstract}
Flavor-changing neutral currents are not present in the Standard Model at tree level and are suppressed in loop processes by the unitarity of the Cabibbo-Kobayashi-Maskawa matrix; the corresponding rates for top quark decay processes are experimentally unobservable. Extensions of the Standard Model can generate new flavor-changing neutral current processes, leading to signals which, if observed, would be unambiguous evidence of new interactions. A data set corresponding to an integrated luminosity of $36.1 \mathrm{fb}^{-1}$ of $p p$ collisions at a center-of-mass energy of $\sqrt{s}=13 \mathrm{TeV}$ recorded with the ATLAS detector at the Large Hadron Collider is used to search for top quarks decaying to up or charm quarks with the emission of a Higgs boson, with subsequent Higgs boson decay to final states with at least one electron or muon. No signal is observed and limits on the branching fractions $\mathcal{B}(t \rightarrow H c)<0.16 \%$ and $\mathcal{B}(t \rightarrow$ $H u)<0.19 \%$ at $95 \%$ confidence level are obtained (with expected limits of $0.15 \%$ in both cases).
\end{abstract}

DOI: 10.1103/PhysRevD.98.032002

\section{INTRODUCTION}

In the Standard Model (SM), the mass eigenstates in the quark sector couple diagonally to the photon, $Z$ boson, and Higgs boson, with the result that quark flavors can only change at tree level by emission of $W^{ \pm}$bosons (charged currents). Although processes that change quark flavors without external emission of $W^{ \pm}$bosons-i.e., flavorchanging neutral currents (FCNC)—occur via loops in the SM, they are suppressed by the Glashow-IliopoulosMaiani mechanism [1]. The decay of a top quark to a Higgs boson and a lighter up-type quark $q(t \rightarrow H q)$ is estimated to have a branching fraction of about $3 \times 10^{-15}$ in the SM [2], which is unobservable with any current or foreseeable data set. An observation of this process with current sensitivity would be unambiguous evidence of physics beyond the Standard Model (BSM). Searches for $t \rightarrow H q$ decays are part of a broader FCNC program that includes searches for $t \gamma q, t Z q$, and $t g q$ interactions [3-7].

Models of BSM physics can feature nontrivial flavor structures that produce tree-level or large effective loopinduced $t H q$ couplings. Tree-level couplings are generic in two-Higgs-doublet models unless discrete symmetries are introduced to forbid them [8], and can also be present in

*Full author list given at the end of the article.

Published by the American Physical Society under the terms of the Creative Commons Attribution 4.0 International license. Further distribution of this work must maintain attribution to the author(s) and the published article's title, journal citation, and DOI. Funded by SCOAP. models with heavy vectorlike quarks [9]. The Cheng-Sher ansatz [10] of off-diagonal light Higgs boson interactions in models with multiple Higgs doublets gives couplings that scale as $\lambda_{t H q} \sim \sqrt{2 m_{t} m_{q}} / v$, where $m_{t}$ is the top quark mass, $m_{q}$ is the light up-type quark mass, and $v$ is the SM Higgs field vacuum expectation value. This would lead to a branching fraction $\mathcal{B}(t \rightarrow H c) \approx 0.15 \%$, close to current experimental bounds. Smaller loop-induced enhancements can be present in two-Higgs-doublet models even without off-diagonal tree-level couplings [11], the minimal supersymmetric Standard Model [12], $R$-parity-violating supersymmetry [13], models with warped extra dimensions [14], and composite Higgs boson models [15]. A summary of expectations for $t \rightarrow H q$ branching fractions in various BSM models can be found in Ref. [16].

The ATLAS and CMS collaborations have carried out searches [17-21] for $t H q$ interactions with 7, 8, and $13 \mathrm{TeV}$ $p p$ collision data from the Large Hadron Collider (LHC). These primarily searched for $t \bar{t}$ production where one top quark decays via $t \rightarrow W b$ and the other decays via $t \rightarrow H q$; the analyses are distinguished by the targeted Higgs boson decay. The results at $13 \mathrm{TeV}$ benefit from the large integrated luminosity delivered by the LHC in 20152016 and from the $t \bar{t}$ cross section being larger at 13 than at $8 \mathrm{TeV}$. Using $13 \mathrm{TeV}$ data, ATLAS obtained $\mathcal{B}(t \rightarrow H c)<0.22 \%$ with $H \rightarrow \gamma \gamma$ decays [20] and CMS obtained $\mathcal{B}(t \rightarrow H c)<0.47 \%$ with $H \rightarrow b \bar{b}$ decays [21], in both cases at $95 \%$ confidence level (C.L.) and assuming $\mathcal{B}(t \rightarrow H u)=0$. Similar limits apply for $\mathcal{B}(t \rightarrow H u)$ assuming $\mathcal{B}(t \rightarrow H c)=0$. 
In this paper, a search for production of $t \bar{t}$ pairs in which one top quark decays via $t \rightarrow H q$ is reported, targeting multilepton final states with either three light leptons $(\ell=e$ or $\mu$ ) or two light leptons of the same electric charge. The multilepton final states can be produced via Higgs boson decays which involve light leptons in the final state through $H \rightarrow W W^{*}, H \rightarrow \tau \tau$ in which both $\tau$-leptons decay leptonically, or $H \rightarrow Z Z^{*}$. A $t \bar{t}$ pair with a $t \rightarrow H q$ decay, followed by $H \rightarrow W W^{*}$, would feature the intermediate state $(W b)\left(W W^{*} q\right)$, which can yield the final states $\ell \ell b 3 q 2 \nu$ or $\ell \ell \ell b q 3 \nu$ ( $q$ representing any quark lighter than the $b$ quark). Events with hadronically decaying $\tau$-lepton candidates are vetoed to avoid overlap with dedicated searches for those Higgs boson decay modes.

The sought-after $t \bar{t}, t \rightarrow H q$ signature with Higgs boson decays into final states with leptons is in many respects similar to the corresponding channel for $t \bar{t} H$ production, although $t \rightarrow H q$ has one fewer $b$-jet and one fewer lightquark jet for the same lepton multiplicity in the absence of additional radiation. The sample of events and background simulations used for the $13 \mathrm{TeV}$ ATLAS search for $\bar{t} \bar{t} H$ production in the multilepton final state [22] is therefore leveraged in this search, similar to the strategy used in the $8 \mathrm{TeV}$ multilepton $t \rightarrow H q$ search [17]. Unlike the $8 \mathrm{TeV}$ search, however, in this analysis multivariate discriminants (boosted decision trees, BDTs) are used, optimized to separate the FCNC signal from SM processes.

The analysis proceeds as follows. The same-charge dilepton and trilepton categories (with no hadronic $\tau$-lepton candidates) from the $13 \mathrm{TeV} t \bar{t} H$ search [22] are used. The same event preselection, calibration, and SM simulation samples are used as in the $t \bar{t} H$ search, but the $t \bar{t} H$ process is now treated as a background and fixed to the expected SM rate. The FCNC processes $t \rightarrow H u$ and $t \rightarrow H c$ are simulated and BDTs are trained to separate these from SM processes in the two categories. Care is taken to account for FCNC signal contamination in control regions which are used to constrain backgrounds arising from lepton production in hadron decays and photon conversions. The results are obtained by fitting the data distributions of the BDT discriminants.

\section{ATLAS DETECTOR AND OBJECT RECONSTRUCTION}

The ATLAS experiment [23] at the LHC is a multipurpose particle detector with a forward-backward symmetric cylindrical geometry and near $4 \pi$ coverage in solid angle. ${ }^{1}$

\footnotetext{
${ }^{1}$ ATLAS uses a right-handed coordinate system with its origin at the nominal interaction point (IP) in the center of the detector and the $z$ axis along the beam pipe. The $x$ axis points from the IP to the center of the LHC ring, and the $y$ axis points upwards. Cylindrical coordinates $(r, \phi)$ are used in the transverse plane, $\phi$ being the azimuthal angle around the $z$ axis. The pseudorapidity is defined in terms of the polar angle $\theta$ as $\eta=-\ln \tan (\theta / 2)$. Angular distance is measured in units of $\Delta R \equiv \sqrt{(\Delta \eta)^{2}+(\Delta \phi)^{2}}$.
}

It consists of an inner tracking detector surrounded by a thin superconducting solenoid providing a $2 \mathrm{~T}$ axial magnetic field, electromagnetic and hadron calorimeters, and a muon spectrometer. The inner tracking detector covers the pseudorapidity range $|\eta|<2.5$. It consists of silicon pixel, silicon microstrip, and transition radiation tracking detectors. A new innermost silicon pixel layer was installed prior to data-taking at $\sqrt{s}=13 \mathrm{TeV}$ [24]. Lead/ liquid-argon (LAr) sampling calorimeters provide electromagnetic (EM) energy measurements with high granularity for $|\eta|<3.2$. A hadron steel/scintillator-tile calorimeter covers the central pseudorapidity range $(|\eta|<1.7)$. The endcap and forward regions are instrumented with LAr calorimeters for both the EM and hadronic energy measurements up to $|\eta|=4.9$. The muon spectrometer surrounds the calorimeters and is based on three large air-core toroidal superconducting magnets with eight coils each. The muon spectrometer includes a system of precision tracking chambers and fast detectors for triggering. A combined hardware and software trigger system is used to select events. The first-level trigger is implemented in hardware and uses a subset of the detector information to reduce the accepted rate to a design maximum of $100 \mathrm{KHz}$. This is followed by a software-based trigger that further reduces the accepted event rate to a sustained rate of about $1 \mathrm{KHz}$.

This analysis uses an integrated luminosity of $36.1 \mathrm{fb}^{-1}$ of $p p$ collision data with $\sqrt{s}=13 \mathrm{TeV}$ collected during 2015 and 2016, corresponding to approximately $30 \times 10^{6}$ $t \bar{t}$ pair production events [25]. The mean number of $p p$ interactions per bunch crossing in the data set is 24 . A full description of the reconstruction and selection of physics objects can be found in Ref. [22], and only a brief description follows.

Events in this analysis are selected using triggers which require the presence of one or two light leptons [26]. The single-lepton triggers have transverse momentum $\left(p_{\mathrm{T}}\right)$ thresholds that vary from 20 to $26 \mathrm{GeV}$ depending on lepton flavor and instantaneous luminosity. In the dilepton triggers the thresholds for the higher- $p_{\mathrm{T}}$ lepton vary from 12 to $22 \mathrm{GeV}$ depending on lepton flavor and instantaneous luminosity.

Muon candidates are formed using inner detector tracks and muon spectrometer tracks, track segments, or for $|\eta|<0.1$, calorimeter signals consistent with the passage of a minimum-ionizing particle. They are required to satisfy $p_{\mathrm{T}}>10 \mathrm{GeV}$ and $|\eta|<2.5$ and to pass loose identification requirements [27], as well as transverse and longitudinal track impact parameter requirements with respect to the primary vertex. The primary vertex in an event is defined as the reconstructed $p p$ collision vertex with the highest $\sum p_{\mathrm{T}}^{2}$ of associated tracks with $p_{\mathrm{T}}>400 \mathrm{MeV}$.

Electron candidates are formed from energy clusters in the electromagnetic calorimeter associated with inner detector tracks. They are required to satisfy $p_{\mathrm{T}}>10 \mathrm{GeV}$ and 
$\left|\eta_{\text {cluster }}\right|<2.47$, excluding the transition region $1.37<$ $\left|\eta_{\text {cluster }}\right|<1.52$ between the barrel and endcap electromagnetic calorimeters. A likelihood discriminant is used to separate isolated prompt electrons from hadronic jets, photon conversions, and nonisolated electrons from hadron decays; two working points of the discriminant (loose and tight [28]) are used in this analysis. Electron candidates must also pass transverse and longitudinal track impact parameter requirements.

Jets are reconstructed using clusters built from energy deposits in the calorimeters [29-31] using the anti- $k_{t}$ algorithm [32,33] with radius parameter $R=0.4$. Considered jets are required to satisfy $p_{\mathrm{T}}>25 \mathrm{GeV}$ and $|\eta|<2.5$. Jets with $p_{\mathrm{T}}<60 \mathrm{GeV}$ and $|\eta|<2.4$ are required to be matched to the primary vertex using their associated inner detector tracks [34]. Jets containing $b$-hadrons ( $b$-jets) are tagged using a multivariate discriminant combining information about secondary vertices, reconstructed decay chains, and impact parameters of tracks associated with the jet $[35,36]$. The working point for $b$-jet identification used in this analysis corresponds to an average efficiency of $70 \%$ for jets containing $b$-hadrons with $p_{\mathrm{T}}>20 \mathrm{GeV}$ and $|\eta|<2.5$ in simulated $t \bar{t}$ events. Jets which contain $c$-hadrons but not $b$-hadrons have approximately a one in twelve probability of being misidentified as $b$-jets; the same probability for lightquark or gluon jets is one in 380 , leading to different responses for the $t \rightarrow H u$ and $t \rightarrow H c$ processes.

Hadronically decaying $\tau$-lepton $\left(\tau_{\text {had }}\right)$ candidates are reconstructed from hadronic jets associated with either one or three inner detector tracks with a total charge of \pm 1 [37,38]. A BDT discriminant is used to distinguish $\tau_{\text {had }}$ candidates from quark- or gluon-initiated jets. A working point with 55\% (40\%) efficiency for one-prong (threeprong) $\tau_{\text {had }}$ decays is used for the veto. Candidates with $p_{\mathrm{T}}>25 \mathrm{GeV}$ and $|\eta|<2.5$ are considered.

The missing transverse momentum, with magnitude $E_{\mathrm{T}}^{\text {miss }}$, is defined as the negative vector sum of the transverse momenta of all calibrated and identified leptons and jets and the remaining unclustered energy of the event, which is estimated from low- $p_{\mathrm{T}}$ tracks associated with the primary vertex but not with any considered lepton or jet [39].

To eliminate ambiguity between reconstructed objects and reduce the fraction of leptons arising from hadron decay, the following additional requirements are imposed: if two electrons are separated by $\Delta R<0.1$, only the higher$p_{\mathrm{T}}$ electron is considered; any electron within $\Delta R=0.1$ of a selected muon is rejected; jets within $\Delta R=0.3$ of a selected electron are removed; any $\tau_{\text {had }}$ candidate within $\Delta R=0.2$ of a selected electron or muon is ignored; and muons must be separated by $\Delta R>\min (0.4,0.04+$ $\left.(10 \mathrm{GeV}) / p_{\mathrm{T}, \mu}\right)$ from a jet surviving the above selection.

To further reject backgrounds, BDTs are trained to discriminate against electrons arising from asymmetric trident processes $e^{ \pm} \rightarrow e^{ \pm} e^{+} e^{-}$in detector material which may induce an apparent change of the electron charge, and against leptons which arise from decays of $b$-hadrons [22]. The former discriminant ("charge misassignment BDT") uses track and calorimeter cluster information; the latter ("nonprompt lepton BDT") uses information about additional particle activity and properties of low- $p_{\mathrm{T}}$ jets formed from tracks near the lepton, including the output of $b$-tagging algorithms run on those jets. Working points with efficiencies of $95 \%(60 \%-98 \%)$ are defined for the charge misassignment (nonprompt lepton) discriminants.

During the initial loose (L) selection of leptons, no isolation, charge misassignment discriminant, or nonprompt lepton discriminant requirements are imposed. Electrons are required to pass the loose electron identification discriminant selection. Two other lepton selections are used in the analysis:

(i) Modified loose $\left(\mathrm{L}^{*}\right)$, in which the lepton must pass calorimeter- and track-based isolation criteria and the nonprompt lepton discriminant selection.

(ii) Tight (T), in which electrons must pass the tight electron identification discriminant selection and the charge misassignment discriminant selection. For muons the $\mathrm{T}$ and $\mathrm{L}^{*}$ selections are identical; this corresponds to the $\mathrm{T}^{*}$ selection of Ref. [22].

\section{SIMULATION, EVENT SELECTION, AND ANALYSIS}

The simulated $p p \rightarrow t \bar{t}, t \rightarrow H q$ signals were generated with next-to-leading-order (NLO) QCD matrix elements computed by MADGRAPH5_AMC@NLO [40], with top quark decays performed by MadSpin [41]; PYTHIA 8 [42] was used for Higgs boson decay, parton showering, hadronization, and underlying-event generation. Either the top quark or antiquark may undergo the FCNC decay in this sample. The total top quark pair production cross section used to normalize the FCNC signal was set to $832_{-46}^{+40} \mathrm{pb}$, as calculated with the ToP++2.0 program at next-to-next-to-leading order in perturbative QCD, including soft-gluon resummation to next-tonext-to-leading-log order [25]. The systematic uncertainties include variation of factorization and renormalization scales as well as uncertainties in parton distribution functions (PDFs) and the QCD coupling $\alpha_{\mathrm{s}}$ [43-46].

The simulations of SM background processes are the same as those used in Ref. [22]. In particular, the major processes $t \bar{t} Z, t \bar{t} W$, and $t \bar{t} H$ were generated at NLO in QCD with MADGRAPH5_AMC@NLO interfaced to PYTHIA 8 for parton showering, hadronization, particle decay, and underlying-event generation. The top quark and Higgs boson masses were set to 172.5 and $125.0 \mathrm{GeV}$, respectively. Higgs boson decay branching fractions were taken from Ref. [47]. In all the preceding samples, the matrix element calculations used the NNPDF 3.0 NLO PDF set [48], while the parton shower calculations used the A14 tune of PYTHIA 8 parameters [49] and the NNPDF 2.3 LO PDF set [46]. Diboson production was generated at NLO in 
QCD with SHERPA [50,51] using the CT10 PDF set [52]. The response of the ATLAS detector to generated events was simulated with a GEANT4-based detector model [53,54] with parametrization of the calorimeter response for some minor backgrounds [55].

The two categories of events used in this analysis, samecharge dilepton ( $2 \ell \mathrm{SS})$ and trilepton $(3 \ell)$ candidates, are selected by the same requirements as in Ref. [22]. In both cases the leptons identified by the trigger must correspond to leptons selected for this analysis, with sufficiently high $p_{\mathrm{T}}$ for the trigger to be fully efficient.

(i) Events in the $2 \ell \mathrm{SS}$ category must have at least four reconstructed jets, of which one or two must be $b$-tagged jets. Exactly two light-lepton candidates meeting L criteria (as described in Sec. II) must be found, along with no $\tau_{\text {had }}$ candidates. The leptons are then also required to pass the $\mathrm{T}$ selection. Both leptons must have $p_{\mathrm{T}}>20 \mathrm{GeV}$ and have the same charge.

(ii) Events in the $3 \ell$ category must have at least two reconstructed jets, of which at least one must be a $b$-tagged jet. Exactly three light-lepton candidates meeting L criteria must be found, along with no $\tau_{\text {had }}$ candidates. The total charge of the leptons must be \pm 1 . Of the three leptons, one (designated $\ell_{0}$ ) is of opposite charge to the other two, and the other two leptons are designated $\ell_{1}$ and $\ell_{2}$ in order of increasing angular separation $\Delta R$ from $\ell_{0}$. The two leptons $\ell_{1}$ and $\ell_{2}$ are required to meet the T criteria and have $p_{\mathrm{T}}>15 \mathrm{GeV}$. The remaining lepton, $\ell_{0}$, is less likely to be nonprompt than either of the same-charge leptons $\ell_{1}$ or $\ell_{2}$. It is correspondingly only required to meet the $\mathrm{L}^{*}$ criteria and to have $p_{\mathrm{T}}>10 \mathrm{GeV}$. To remove contamination from hadron decay chains including $\ell^{+} \ell^{\prime-}$, both invariant masses $m\left(\ell_{0} \ell_{1}\right)$ and $m\left(\ell_{0} \ell_{2}\right)$ must exceed $12 \mathrm{GeV}$. To remove contamination from $t \bar{t} Z$, a $Z$ boson veto is imposed: $\left|m\left(\ell^{+} \ell^{-}\right)-91.2 \mathrm{GeV}\right|>$ $10 \mathrm{GeV}$ for every opposite-charge lepton pair of the same flavor $\left(e^{+} e^{-}\right.$or $\left.\mu^{+} \mu^{-}\right)$. Finally, contamination from $Z \rightarrow \ell \ell \gamma^{(*)} \rightarrow \ell \ell \ell^{\prime}\left(\ell^{\prime}\right)$, where one lepton has low momentum and is not reconstructed, is removed by requiring $|m(3 \ell)-91.2 \mathrm{GeV}|>10 \mathrm{GeV}$.

After these selections, the $t \rightarrow H q$ signal is dominated by $H \rightarrow W W^{*}$ ( $85 \%$ of the $2 \ell \mathrm{SS}$ and $71 \%$ of the $3 \ell$ category) with subleading contributions from $H \rightarrow \tau \tau(12 \%$ and $16 \%$ respectively) and $H \rightarrow Z Z^{*}$ (2\% and 9\% respectively). The fraction of $t \bar{t}$ events with a consequent $t \rightarrow H q$ decay which are expected to be reconstructed and selected is $5.1 \times 10^{-4}$ $\left(2.6 \times 10^{-4}\right)$ for the $2 \ell \mathrm{SS}(3 \ell)$ category. $^{2}$

Following the initial selections, the largest sources of background are those arising from nonprompt leptons

\footnotetext{
${ }^{2}$ These values include the effects of selection acceptance, detector efficiency, and decay branching fractions.
}

(from hadron decays, photon conversions, and charge misassignment), mainly from $t \bar{t}$ decays, and prompt lepton backgrounds from $t \bar{t} V$ production $(V=W$ or $Z)$ with leptonic decays of the vector boson. Further BDT discriminants are trained to separate the FCNC signal from these two background sources.

Inputs to the BDTs include lepton flavor and kinematic observables, jet properties including whether they are $b$ tagged, angular separations between objects, the $E_{\mathrm{T}}^{\text {miss }}$, and the quantity $m_{\mathrm{eff}} \equiv E_{\mathrm{T}}^{\text {miss }}+H_{\mathrm{T}}$, where $H_{\mathrm{T}}$ is the scalar sum of the $p_{\mathrm{T}}$ of leptons and jets in the event. Signal events can be distinguished from $t \bar{t} V$ and nonprompt lepton background by having only one true $b$-jet and being relatively soft events with low $m_{\text {eff }}$ and $H_{\mathrm{T}}$. The spin correlation in the dominant $H \rightarrow W W^{*}$ decay, and the presence of an off-mass-shell $W^{*}$ boson, also yields a distinct signature where, in both categories, one lepton often has low $p_{\mathrm{T}}$, and in the $3 \ell$ category, both $m\left(\ell_{0} \ell_{1}\right)$ and $\Delta R\left(\ell_{0}, \ell_{1}\right)$ are small.

The variables used in the training of the BDTs in the two categories are shown in Table I, and example distributions are shown in Fig. 1. Good agreement is observed between the data and expected background distributions in each variable. In the $2 \ell \mathrm{SS}$ category, the variables that most

TABLE I. Variables used to construct the BDT discriminants for the $2 \ell \mathrm{SS}$ and $3 \ell$ categories. The symbol " $x$ " indicates that this variable is used in the respective BDT. The "best $Z$ candidate" is the opposite-charge lepton pair with same flavor with invariant mass closest to $91.2 \mathrm{GeV}$; if no such pair exists, zero is assigned for the invariant mass.

\begin{tabular}{lcc}
\hline \hline Variable & $2 \ell \mathrm{SS}$ & $3 \ell$ \\
\hline$p_{\mathrm{T}}$ of higher- $p_{\mathrm{T}}$ lepton & $\times$ & \\
$p_{\mathrm{T}}$ of lower- $p_{\mathrm{T}}$ lepton & $\times$ & \\
$p_{\mathrm{T}}$ of lepton $\ell_{0}$ & & $\times$ \\
$p_{\mathrm{T}}$ of lepton $\ell_{1}$ & & $\times$ \\
$p_{\mathrm{T}}$ of lepton $\ell_{2}$ & $\times$ & $\times$ \\
Dilepton invariant masses (all combinations) & & $\times$ \\
Trilepton invariant mass & & $\times$ \\
Best $Z$ candidate invariant mass & $\times$ & \\
Maximum lepton $|\eta|$ & $\times$ & \\
Lepton flavor & $\times$ & $\times$ \\
Number of jets & $\times$ & $\times$ \\
Number of $b$-tagged jets & & $\times$ \\
$p_{\mathrm{T}}$ of highest- $p_{\mathrm{T}}$ jet & & $\times$ \\
$p_{\mathrm{T}}$ of second highest- $p_{\mathrm{T}}$ jet & & $\times$ \\
$p_{\mathrm{T}}$ of highest- $p_{\mathrm{T}} b$-tagged jet & & $\times$ \\
$\Delta R\left(\ell_{0}, \ell_{1}\right)$ & & $\times$ \\
$\Delta R\left(\ell_{0}, \ell_{2}\right)$ & $\times$ & \\
$\Delta R$ (higher- $p_{\mathrm{T}}$ lepton, closest jet $)$ & $\times$ & \\
$\Delta R\left(\right.$ lower- $p_{\mathrm{T}}$ lepton, closest jet $)$ & & $\times$ \\
$\Delta R\left(\ell_{1}\right.$, closest jet $)$ & $\times$ \\
Smallest $\Delta R\left(\ell_{0}, b\right.$-tagged jet $)$ & $\times$ \\
$E_{\mathrm{T}}^{\text {miss }}$ & $\times$ \\
$m_{\mathrm{eff}}$ & & $\times$ \\
\hline \hline
\end{tabular}



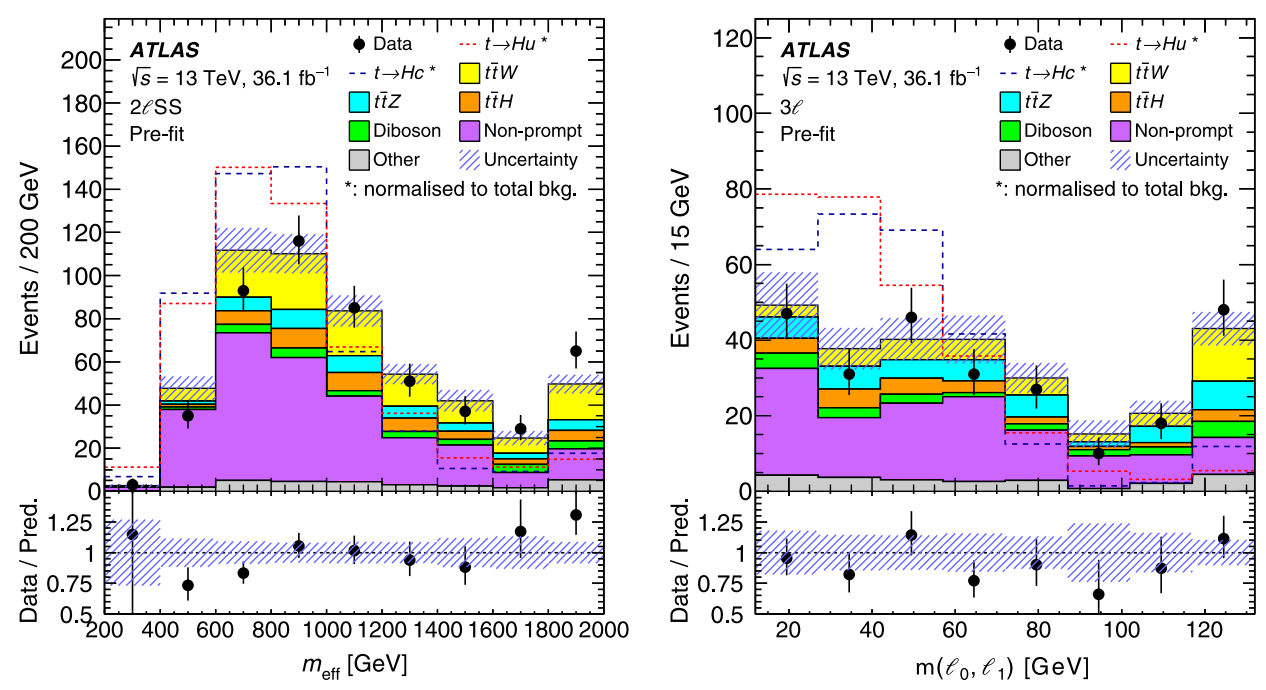

FIG. 1. Examples of distributions of variables separating FCNC signals from SM background: Left: the quantity $m_{\text {eff }}$ (defined in the text) in the $2 \ell \mathrm{SS}$ category. Right: the invariant mass $m\left(\ell_{0}, \ell_{1}\right)$ of the opposite-charge lepton pair with the smaller angular separation $\Delta R$ in the $3 \ell$ category. The bottom panels show the ratio of the observed data yields in each bin to the SM prediction. The hashed bands indicate the total uncertainty in the SM prediction. The FCNC signal distributions are shown as open histograms, normalized to the same total yield as the SM backgrounds. The rightmost bins include overflow.

strongly separate $t \rightarrow H q$ from the nonprompt lepton background are the number of $b$-jets, the $p_{\mathrm{T}}$ of the lower- $p_{\mathrm{T}}$ lepton, and the angular separation $\Delta R$ of the lower- $p_{\mathrm{T}}$ lepton from the closest jet. The separation from $t \bar{t} V$ processes comes mainly from the number of $b$-jets, $m_{\mathrm{eff}}, E_{\mathrm{T}}^{\mathrm{miss}}$, and the $p_{\mathrm{T}}$ of the higher- $p_{\mathrm{T}}$ lepton. In the $3 \ell$ category the strongest separation from nonprompt lepton backgrounds comes from $\Delta R\left(\ell_{0}, \ell_{1}\right)$, the invariant masses $m\left(\ell_{0}, \ell_{1}\right)$ and $m\left(\ell_{0}, \ell_{2}\right)$, the $p_{\mathrm{T}}$ of the highest- $p_{\mathrm{T}}$ lepton, and the invariant mass of the opposite-charge, same-flavor lepton pair with mass closest to that of the $Z$ boson. The strongest separation from $t \bar{t} V$ comes from the invariant masses $m\left(\ell_{0}, \ell_{1}\right)$ and $m\left(\ell_{0}, \ell_{2}\right), m_{\text {eff }}$, the number of $b$-jets, and the invariant mass of the three leptons. In both the $2 \ell \mathrm{SS}$ and $3 e$ categories and against both backgrounds, better separation is achieved for $t \rightarrow H u$ than $t \rightarrow H c$ signals, as the latter is more likely to have a second $b$-tagged jet arising from the hadronization products of the charm quark. In the $2 \ell$ SS category, $t \rightarrow H u$ and $t \rightarrow H c$ are sufficiently similar that only one discriminant is trained for the two decay modes. In the $3 l$ category the two signals are treated separately.

In each case, two separate discriminants are trained: one to separate $t \rightarrow H q$ from nonprompt leptons and one to separate $t \rightarrow H q$ from $t \bar{t} V$ processes. The former is designated $\operatorname{BDT}(t \bar{t}, X)$ and the latter $\operatorname{BDT}(t \bar{t} V, X)$ for a given category/signal choice $X$ (i.e., $2 \ell \mathrm{SS}, 3 \ell t \rightarrow H u$, or $3 \ell t \rightarrow H c$ ). The expected distributions for backgrounds with nonprompt leptons are obtained using the procedures described in Sec. IV. The two discriminants are combined into a single discriminant, designated $\operatorname{BDT}(X)$, via a linear combination that yields the best expected limit on the FCNC branching fraction.

The BDT discriminant outputs in the $2 \ell \mathrm{SS}$ and $3 \ell$ categories are binned into six or four bins, respectively, with bin widths optimized to provide the best expected limits. Every bin contains a roughly equal number of signal events. The bin boundaries used for the fits to $t \rightarrow H u$ and $t \rightarrow H c$ signals in the $2 \ell$ SS category are optimized separately, although the discriminant used is the same for the two decays.

\section{BACKGROUND ESTIMATION}

The estimation of rates and kinematic distributions of SM processes that form backgrounds with prompt leptons in the signal categories is performed using simulation. The processes considered include the following:

(i) $t \bar{t} W, t \bar{t}\left(Z / \gamma^{*} \rightarrow \ell \ell\right), t \bar{t} H$, and $t \bar{t} W W$;

(ii) $t \bar{t} t$ and $t \bar{t} t \bar{t}$;

(iii) single top quark production in the $s$ - and $t$-channels, $t W, t Z, t W Z, t H b$, and $t H W$

(iv) production of two or three $W$ or $Z / \gamma^{*}$ bosons. Details of the simulations used are given in Ref. [22].

The estimation of the nonprompt lepton backgrounds, including the contribution from charge-misassigned electrons, uses data-driven methods following Ref. [22]. One major modification to the treatment of leptons from hadron decays and conversions is made, arising from the lower expected jet multiplicity in $t \rightarrow H q$ events compared to $t \bar{t} H$ production. A summary of the procedure is given below. The self-consistency of the methods is checked by 
predicting the nonprompt lepton yields in simulated SM $t \bar{t}$ and $t \bar{t} \gamma$ events; good agreement is observed.

Rates for electron charge misassignment as functions of electron $p_{\mathrm{T}}$ and $|\eta|$ are determined from a sample of $Z \rightarrow e e$ events where both electrons are reconstructed with the same charge. The contribution of these events to the $2 \ell \mathrm{SS}$ signal region is determined by applying these misassignment rates to events selected with the $2 \ell \mathrm{SS}$ selection criteria except requiring opposite-charge leptons instead of same-charge leptons.

The estimation of nonprompt lepton background contributions other than electron charge misassignment is performed using the so-called matrix method [56,57]. This technique uses control regions with the same kinematic properties as the signal region, but with changed lepton identification criteria that enhance the nonprompt lepton contribution, to statistically estimate the fraction of signal region events that involve nonprompt leptons. Lepton candidates that meet $\mathrm{L}$, but not $\mathrm{T}$, identification criteria $(\overline{\mathrm{T}})$ are much more likely to be of nonprompt origin than those that meet $\mathrm{T}$ criteria; if the probabilities ("efficiencies") of prompt and nonprompt leptons to be identified as $\overline{\mathrm{T}}$ or $\mathrm{T}$ are known, the fraction of tight-lepton events of nonprompt origin can be determined by solving a system of equations. The matrix method can be applied to estimate contributions arising from multiple nonprompt leptons in the same event. In this analysis, the two leptons in the $2 \ell \mathrm{SS}$ category events, and the two same-charge leptons in the $3 \ell$ category events, are analyzed for a nonprompt contribution. The opposite-sign lepton $\ell_{0}$ in $3 \ell$ events is found in simulation to be prompt $97 \%$ of the time, so the matrix method is not applied to that lepton.

The nonprompt efficiencies are measured in control regions with the same selection criteria as the $2 \ell \mathrm{SS}$ signal category except that the number of jets must be two or three, one lepton need only satisfy L criteria, and the lower$p_{\mathrm{T}}$ lepton is only required to have $p_{\mathrm{T}}>15 \mathrm{GeV}$. These are then separated into TT or TT events. The expected number of events from SM processes with only prompt leptons in these regions is determined from simulation and subtracted from the observed number of events, giving a yield of nonprompt lepton events which is then used to determine the nonprompt efficiencies. In the case of a nonzero $t \rightarrow$ $\mathrm{Hq}$ branching fraction, a significant fraction of the signal will be reconstructed in these control regions. This will act as an additional source of prompt leptons which is not accounted for in the SM prediction and will bias the nominal efficiencies which are determined assuming zero signal contribution. For $\mathcal{B}(t \rightarrow H q)=0.2 \%$, the FCNC process would contribute approximately $30 \%$ of the prompt lepton contribution in the low-jet-multiplicity control regions with $\mathrm{T}$ leptons. This effect is accounted for in two ways. First, the nonprompt efficiencies are derived under the two hypotheses $\mathcal{B}(t \rightarrow H q)=0$ and $\mathcal{B}(t \rightarrow H q)=0.2 \%$ and both values are used to predict the yield of nonprompt leptons in the signal categories; the two hypotheses result in nonprompt yields differing by $\approx 40 \%$ for the $2 \ell \mathrm{SS}$ and $\approx 30 \%$ for the $3 \ell$ category. This overall normalization correction from possible signal contamination is then scaled proportionally to the FCNC branching fraction in the fit. Second, the change in the shape of the FCNC discriminant response for the nonprompt background in the signal regions under the two hypotheses is derived. The difference is assigned as a systematic uncertainty on the nonprompt background discriminant shape.

The separation of FCNC signal from nonprompt lepton background by the $\operatorname{BDT}(t \bar{t}, X)$ discriminants is sufficiently strong that the impact of these systematic uncertainties in the nonprompt background estimate on the signal extraction is small. Tests with MC simulation indicate that the procedure correctly recovers the branching fraction of injected FCNC signals.

\section{SYSTEMATIC UNCERTAINTIES}

The same model of systematic uncertainties in background processes (including $t \bar{t} H$ ) is used as in Ref. [22], with the additional normalization and BDT shape uncertainties in nonprompt lepton backgrounds described in Sec. IV. As the measured $t \bar{t} H$ cross section is compatible with the SM predictions, the SM rate is assumed with appropriate theoretical uncertainties. Acceptance uncertainties from the choice of parton distribution functions and QCD scale for the major backgrounds simulated with MADGRAPH5_AMC@NLO are calculated using SYSCALC [58].

The $t \rightarrow H q$ signal processes are subject to their own theoretical uncertainties, primarily in the modeling of the parent $t \bar{t}$ system. Systematic uncertainties are assigned for the $t \bar{t}$ cross section, the variation of BDT response with the choice of renormalization and factorization scale, the modeling of parton showers, the event generator, and the amount of initial/final-state radiation.

The systematic uncertainty model includes components from

(i) light lepton, $\tau_{\text {had }}$, and jet selection and energy/ momentum scale and $E_{\mathrm{T}}^{\text {miss }}$ modeling;

(ii) $b$-jet tagging efficiency and the probability for $c$-jets and light-quark or gluon jets to be misidentified as $b$-jets;

(iii) the cross section and MC modeling of simulated backgrounds and signals;

(iv) the statistical uncertainties in the control regions for nonprompt lepton backgrounds, the matrix method efficiencies, and the applicability to the $2 \ell \mathrm{SS}$ and $3 \ell$ category events of the matrix method efficiencies derived at low jet multiplicity in same-charge dilepton events;

(v) electron charge misassignment;

(vi) $p p$ integrated luminosity (determined using a methodology similar to that described in Ref. [59]); 
(vii) modeling of multiple $p p$ interactions per bunch crossing.

The background-related systematic uncertainties with the largest impact on the final result are found to be those associated with the statistical uncertainty in the nonprompt lepton background estimation, the nonprompt lepton efficiencies used in the matrix method, and the cross section for diboson production in association with $b$-quarks. Excluding the correction for signal contributions to nonprompt lepton control regions, systematic uncertainties on the background lead to an uncertainty in the determined signal decay branching fractions of $0.04 \%$. Systematic uncertainties in the signal processes are primarily associated with the matching of matrix element calculations with parton shower algorithms and different choices of parton shower algorithms. The relative systematic uncertainty in the signal yield prediction for a given signal branching fraction is $8 \%$. The uncertainty in the background estimate due to the signal contributions in nonprompt lepton control regions depends on the signal decay branching fraction; for a true branching fraction of $0.2 \%$, the corresponding systematic uncertainty on the determined branching fraction is $0.02 \%$.

\section{RESULTS}

Binned maximum-likelihood fits to the distributions of the $2 \ell \mathrm{SS}$ and $3 \ell \mathrm{FCNC}$ discriminants are performed to extract the best-fit values of the $t \rightarrow H q$ branching fractions. The profile likelihood technique is used, in which systematic uncertainties are modeled as nuisance parameters $\vec{\theta}$ which are allowed to vary in the fit, constrained by Gaussian or log-normal probability density penalty functions multiplying the likelihood function $\mathcal{L}$. The test statistic $q_{\mathcal{B}}$ is obtained from the profile log-likelihood ratio as $q_{\mathcal{B}} \equiv-2 \ln \Lambda_{\mathcal{B}}=-2 \ln [\mathcal{L}(\mathcal{B}, \hat{\vec{\theta}}) / \mathcal{L}(\hat{\mathcal{B}}, \hat{\vec{\theta}})]$, where $\hat{\mathcal{B}}$ and $\hat{\vec{\theta}}$ are the $t \rightarrow H q$ branching fraction and nuisance parameter values that give the global maximum likelihood and $\hat{\vec{\theta}}$ are the nuisance parameter values which maximize $\mathcal{L}$ for a given branching fraction $\mathcal{B}$. The uncertainties in the best-fit branching fraction value $\hat{\mathcal{B}}$ are determined by the variation of $q_{\mathcal{B}}$ by one unit from its minimum, and the distribution of $q_{\mathcal{B}}$ is used to set $95 \%$ C.L. upper limits on the branching fractions $\mathcal{B}(t \rightarrow H q)$ using the $\mathrm{CL}_{\mathrm{s}}$ method [60]. Due to the near-degeneracy of the BDT response to $t \rightarrow H u$ and $t \rightarrow H c$ signals, during the fits, one of these branching fractions is set to zero while the other is permitted to float. Expected and observed yields of events in the signal categories, before and after the nuisance parameters are adjusted in the fits, are shown in Table II. The distributions of the FCNC discriminant for the data and the best-fit signal-plus-background models are shown in Fig. 2.

The results of the fits are shown in Tables III and IV. The best-fit branching fractions are compatible with zero, and 95\% C.L. upper limits are set, as shown in Fig. 3. Statistical uncertainties are dominant in the result. No variations of the nuisance parameters by more than $1 \sigma$ of the prior systematic uncertainty are observed; the largest variations are observed in the nuisance parameters associated with statistical uncertainties in the nonprompt lepton background estimate and in one of the normalization systematic uncertainties in the $3 \ell$ nonprompt lepton background.

To confirm the self-consistency of the nonprompt lepton background estimate, a number of checks were performed. There is no evidence of a BDT response shape distortion in the nonprompt lepton background estimate during the fit

TABLE II. Expected SM background (including nonprompt leptons), FCNC contributions, and observed data yields in the signal categories. The FCNC contribution shown in the "prefit" rows is the expected yield for a $0.2 \%$ branching fraction, and the result in the "postfit" rows is the best-fit yield from the combined fit of the $2 \ell \mathrm{SS}$ and $3 \ell$ categories. The uncertainties shown for the FCNC yields reflect systematic uncertainties given a specific branching fraction (prefit) or the uncertainties in the yield from the full fit (postfit). SM backgrounds are determined as described in Sec. IV. The nonprompt lepton background estimate includes electron charge misassignment. The nonprompt lepton component of the prefit SM background is determined assuming zero FCNC branching fraction; the postfit nonprompt lepton background yield includes the effect of nonzero FCNC branching fraction.

\begin{tabular}{|c|c|c|c|c|c|c|c|c|c|}
\hline \multicolumn{2}{|c|}{ Category } & Nonprompt leptons & $t \bar{t} V$ & $t \bar{t} H$ & Diboson & Other prompt SM & Total SM & FCNC & Data \\
\hline \multicolumn{10}{|c|}{$t \rightarrow H u$} \\
\hline \multirow{2}{*}{$2 \ell \mathrm{SS}$} & prefit & $266 \pm 40$ & $165 \pm 19$ & $43 \pm 4$ & $25 \pm 15$ & $28 \pm 6$ & $526 \pm 39$ & $61 \pm 13$ & \multirow{2}{*}{514} \\
\hline & postfit & $240 \pm 37$ & $167 \pm 18$ & $43 \pm 4$ & $24 \pm 14$ & $28 \pm 6$ & $502 \pm 33$ & $13 \pm 21$ & \\
\hline \multirow{2}{*}{$3 e$} & prefit & $126 \pm 31$ & $84 \pm 8$ & $23 \pm 3$ & $20 \pm 11$ & $24 \pm 5$ & $276 \pm 33$ & $32 \pm 6$ & \multirow{2}{*}{258} \\
\hline & postfit & $104 \pm 20$ & $84 \pm 8$ & $23 \pm 3$ & $19 \pm 10$ & $24 \pm 5$ & $254 \pm 18$ & $7 \pm 11$ & \\
\hline \multicolumn{10}{|c|}{$t \rightarrow H c$} \\
\hline \multirow{2}{*}{$2 \ell \mathrm{SS}$} & prefit & $266 \pm 40$ & $165 \pm 19$ & $43 \pm 4$ & $25 \pm 15$ & $28 \pm 6$ & $526 \pm 39$ & $62 \pm 13$ & \multirow{2}{*}{514} \\
\hline & postfit & $264 \pm 41$ & $165 \pm 18$ & $42 \pm 4$ & $20 \pm 11$ & $28 \pm 6$ & $520 \pm 36$ & $-3 \pm 25$ & \\
\hline \multirow{2}{*}{$3 e$} & prefit & $126 \pm 31$ & $84 \pm 8$ & $23 \pm 3$ & $20 \pm 11$ & $24 \pm 5$ & $276 \pm 33$ & $30 \pm 6$ & \multirow{2}{*}{258} \\
\hline & postfit & $116 \pm 21$ & $84 \pm 8$ & $23 \pm 3$ & $15 \pm 8$ & $23 \pm 5$ & $262 \pm 19$ & $-1 \pm 12$ & \\
\hline
\end{tabular}



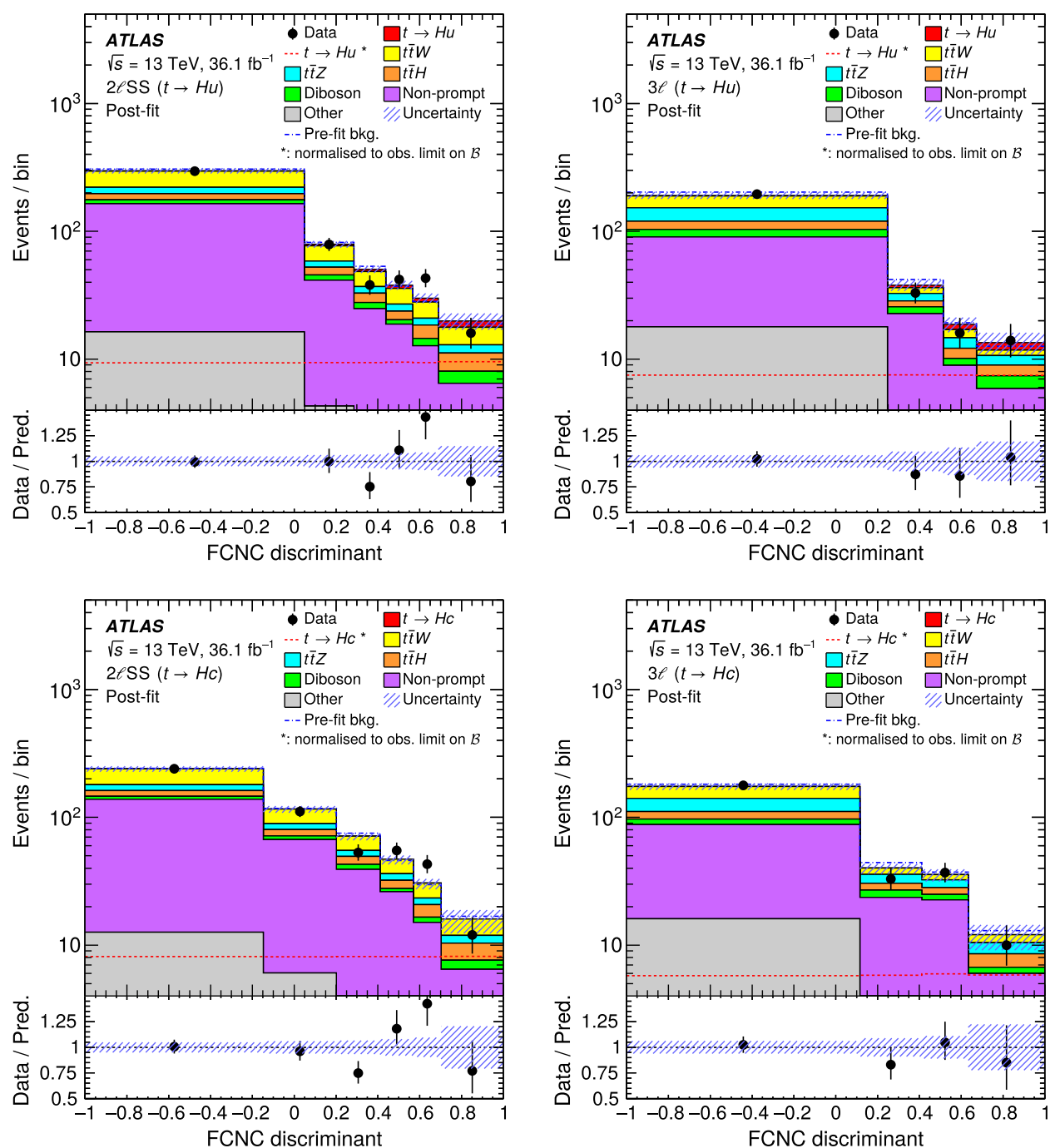

FIG. 2. Distributions of the FCNC signal discriminants for (top) $t \rightarrow H u$, (bottom) $t \rightarrow H c$ signals in (left) $2 \ell \mathrm{SS}$, (right) $3 \ell$ categories. In the $t \rightarrow H u$ fit, $\mathcal{B}(t \rightarrow H c)$ is set to zero, and vice versa. The binning, which is that used in the fit, is chosen so as to have roughly equal signal yields in each bin. The FCNC signals, normalized to their best-fit branching fractions from the combined fit to the $2 \ell$ SS and $3 \ell$ categories, are shown as filled red histograms stacked above the background components; only $t \rightarrow H u$ is large enough to be visible. The hashed band indicates the total uncertainty in the signal-plus-background prediction, including the statistical uncertainty in the bestfit FCNC signal. The dashed red lines show the expected contribution of the respective FCNC decay with the 95\% C.L. upper limit branching fraction $(0.19 \%$ for $t \rightarrow H u, 0.16 \%$ for $t \rightarrow H c)$.

TABLE III. Best-fit values and 95\% C.L. upper limits for $\mathcal{B}(t \rightarrow H u)$, assuming $\mathcal{B}(t \rightarrow H c)=0$. The "stat + syst" columns show the full result allowing all systematic uncertainty nuisance parameters to float in the fit, while the "stat" columns show the result with systematic uncertainty nuisance parameters fixed to their values at the global best-fit point.

\begin{tabular}{lccccc}
\hline \hline & \multicolumn{2}{c}{ Best-fit } & & \multicolumn{2}{c}{ Observed (expected) } \\
& \multicolumn{2}{c}{$\mathcal{B}(t \rightarrow H u)[\%]$} & & Upper limit on $\mathcal{B}(t \rightarrow H u)[\%]$ \\
\cline { 2 - 3 } \cline { 5 - 6 } & stat & stat + syst & stat & stat + syst \\
\hline $2 \ell$ SS & $0.08_{-0.08}^{+0.08}$ & $0.08_{-0.10}^{+0.11}$ & & $0.23(0.15)$ & $0.28(0.21)$ \\
$3 \ell$ & $0.01_{-0.08}^{+0.09}$ & $0.01_{-0.09}^{+0.10}$ & & $0.20(0.18)$ & $0.22(0.21)$ \\
Combined & $0.04_{-0.06}^{+0.06}$ & $0.04_{-0.07}^{+0.08}$ & & $0.17(0.12)$ & $0.19(0.15)$ \\
\hline \hline
\end{tabular}

TABLE IV. Best-fit values and 95\% C.L. upper limits for $\mathcal{B}(t \rightarrow H c)$, assuming $\mathcal{B}(t \rightarrow H u)=0$. The "stat + syst" columns show the full result allowing all systematic uncertainty nuisance parameters to float in the fit, while the "stat" columns show the result with systematic uncertainty nuisance parameters fixed to their values at the global best-fit point.

\begin{tabular}{lrrrrr}
\hline \hline & \multicolumn{2}{c}{ Best-fit } & & \multicolumn{2}{c}{$\begin{array}{c}\text { Observed (expected) } \\
\text { Upper Limit on }\end{array}$} \\
& \multicolumn{2}{c}{$\mathcal{B}(t \rightarrow H c)[\%]$} & & \multicolumn{2}{c}{$\mathcal{B}(t \rightarrow H c)[\%]$} \\
\cline { 2 - 3 } \cline { 5 - 7 } & \multicolumn{1}{c}{ stat } & stat + syst & & stat & stat + syst \\
\hline $2 \ell$ SS & $0.05_{-0.08}^{+0.08}$ & $0.05_{-0.10}^{+0.11}$ & & $0.22(0.15)$ & $0.25(0.20)$ \\
$3 \ell$ & $-0.09_{-0.09}^{+0.10}$ & $-0.09_{-0.11}^{+0.11}$ & & $0.19(0.23)$ & $0.20(0.25)$ \\
Combined & $-0.01_{-0.06}^{+0.06}$ & $-0.01_{-0.08}^{+0.08}$ & & $0.15(0.13)$ & $0.16(0.15)$ \\
\hline \hline
\end{tabular}




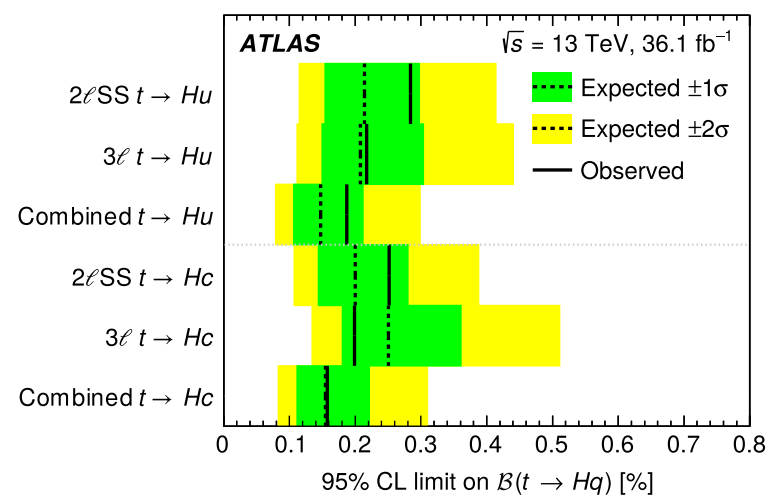

FIG. 3. Observed and expected 95\% C.L. upper limits on $\mathcal{B}(t \rightarrow H u)$ and $\mathcal{B}(t \rightarrow H c)$. In each case, the other FCNC decay is assumed to have zero branching fraction. The individual results from each signal category are shown as well as the combination.

which would be consistent with a FCNC signal. In addition, a second fit that decorrelates FCNC contributions in the signal categories from their impact on the nonprompt lepton efficiency estimates finds best-fit branching fractions consistent with those of the nominal fit.

\section{CONCLUSION}

Any observable branching fraction for the FCNC decays $t \rightarrow H q$ would indicate new physics beyond the Standard Model. A search for $t \bar{t}$ production events in which one top quark or antiquark undergoes a $t \rightarrow H q$ decay was carried out with an integrated luminosity of $36.1 \mathrm{fb}^{-1}$ of $p p$ collision data with $\sqrt{s}=13 \mathrm{TeV}$ collected in 2015 and 2016 using the ATLAS detector at the LHC. Two final states are targeted: two same-charge light leptons with four or more jets, and three light leptons with two or more jets. These are sensitive primarily to $H \rightarrow W W^{*}$ decays, with subleading contributions from $H \rightarrow \tau \tau$ and $H \rightarrow Z Z^{*}$. Specialized boosted decision trees using the kinematic properties of the final-state particles are used to distinguish FCNC signals from nonprompt lepton backgrounds and from $t \bar{t} W$ and $t \bar{t} Z$ production. Potential contamination from FCNC signal in the nonprompt lepton background control regions is treated in a self-consistent manner. No evidence of FCNC decays is found and the upper limits set on the branching fractions are $\mathcal{B}(t \rightarrow H c)<0.16 \%$ and $\mathcal{B}(t \rightarrow H u)<0.19 \%$ at $95 \%$ C.L.

\section{ACKNOWLEDGMENTS}

We thank CERN for the very successful operation of the LHC, as well as the support staff from our institutions without whom ATLAS could not be operated efficiently. We acknowledge the support of ANPCyT, Argentina; YerPhI, Armenia; ARC, Australia; BMWFW and FWF, Austria; ANAS, Azerbaijan; SSTC, Belarus; CNPq and FAPESP, Brazil; NSERC, NRC and CFI, Canada; CERN; CONICYT, Chile; CAS, MOST and NSFC, China; COLCIENCIAS, Colombia; MSMT CR, MPO CR and VSC CR, Czech Republic; DNRF and DNSRC, Denmark; IN2P3-CNRS, CEA-DRF/IRFU, France; SRNSFG, Georgia; BMBF, HGF, and MPG, Germany; GSRT, Greece; RGC, Hong Kong SAR, China; ISF, I-CORE and Benoziyo Center, Israel; INFN, Italy; MEXT and JSPS, Japan; CNRST, Morocco; NWO, Netherlands; RCN, Norway; MNiSW and NCN, Poland; FCT, Portugal; MNE/IFA, Romania; MES of Russia and NRC KI, Russian Federation; JINR; MESTD, Serbia; MSSR, Slovakia; ARRS and MIZŠ, Slovenia; DST/NRF, South Africa; MINECO, Spain; SRC and Wallenberg Foundation, Sweden; SERI, SNSF and Cantons of Bern and Geneva, Switzerland; MOST, Taiwan; TAEK, Turkey; STFC, United Kingdom; DOE and NSF, United States of America. In addition, individual groups and members have received support from BCKDF, the Canada Council, CANARIE, CRC, Compute Canada, FQRNT, and the Ontario Innovation Trust, Canada; EPLANET, ERC, ERDF, FP7, Horizon 2020 and Marie Skłodowska-Curie Actions, European Union; Investissements d'Avenir Labex and Idex, ANR, Région Auvergne and Fondation Partager le Savoir, France; DFG and AvH Foundation, Germany; Herakleitos, Thales and Aristeia programmes co-financed by EU-ESF and the Greek NSRF; BSF, GIF and Minerva, Israel; BRF, Norway; CERCA Programme Generalitat de Catalunya, Generalitat Valenciana, Spain; the Royal Society and Leverhulme Trust, United Kingdom. The crucial computing support from all WLCG partners is acknowledged gratefully, in particular from CERN, the ATLAS Tier-1 facilities at TRIUMF (Canada), NDGF (Denmark, Norway, Sweden), CC-IN2P3 (France), KIT/ GridKA (Germany), INFN-CNAF (Italy), NL-T1 (Netherlands), PIC (Spain), ASGC (Taiwan), RAL (UK) and BNL (USA), the Tier-2 facilities worldwide and large non-WLCG resource providers. Major contributors of computing resources are listed in Ref. [61].
[1] S. L. Glashow, J. Iliopoulos, and L. Maiani, Weak Interactions with lepton-hadron symmetry, Phys. Rev. D 2, 1285 (1970).
[2] J. A. Aguilar-Saavedra, Top flavor-changing neutral interactions: Theoretical expectations and experimental detection, Acta Phys. Pol. B 35, 2695 (2004). 
[3] CMS Collaboration, Search for anomalous single top quark production in association with a photon in pp collisions at $\sqrt{s}=8 \mathrm{TeV}$, J. High Energy Phys. 04 (2016) 035.

[4] ATLAS Collaboration, Search for flavour-changing neutral current top-quark decays to $\mathrm{qZ}$ in $\mathrm{pp}$ collision data collected with the ATLAS detector at $\sqrt{s}=8 \mathrm{TeV}$, Eur. Phys. J. C 76, 12 (2016).

[5] CMS Collaboration, Search for associated production of a Z boson with a single top quark and for $\mathrm{tZ}$ flavour-changing interactions in pp collisions at $\sqrt{s}=8 \mathrm{TeV}$, J. High Energy Phys. 07 (2017) 003.

[6] ATLAS Collaboration, Search for single top-quark production via flavour-changing neutral currents at $8 \mathrm{TeV}$ with the ATLAS detector, Eur. Phys. J. C 76, 55 (2016).

[7] CMS Collaboration, Search for anomalous Wtb couplings and flavour-changing neutral currents in t-channel single top quark production in pp collisions at $\sqrt{s}=7$ and $8 \mathrm{TeV}$, J. High Energy Phys. 02 (2017) 028.

[8] S. L. Glashow and S. Weinberg, Natural conservation laws for neutral currents, Phys. Rev. D 15, 1958 (1977).

[9] F. del Aguila and J. Cortes, A new model of weak $C P$ violation, Phys. Lett. B 156, 243 (1985).

[10] T. P. Cheng and M. Sher, Mass-matrix ansatz and flavor nonconservation in models with multiple Higgs doublets, Phys. Rev. D 35, 3484 (1987).

[11] S. Bejar, J. Guasch, and J. Sola, Loop induced flavor changing neutral decays of the top quark in a general two Higgs doublet model, Nucl. Phys. B600, 21 (2001).

[12] J. Guasch and J. Sola, FCNC top quark decays: A door to SUSY physics in high luminosity colliders?, Nucl. Phys. B562, 3 (1999).

[13] J. M. Yang, B.-L. Young, and X. Zhang, Flavor-changing top quark decays in $R$-parity-violating supersymmetric models, Phys. Rev. D 58, 055001 (1998).

[14] K. Agashe, G. Perez, and A. Soni, Collider signals of top quark flavor violation from a warped extra dimension, Phys. Rev. D 75, 015002 (2007).

[15] K. Agashe and R. Contino, Composite Higgs-mediated flavor-changing neutral current, Phys. Rev. D 80, 075016 (2009).

[16] K. Agashe et al., Top quark working group report, arXiv: 1311.2028.

[17] ATLAS Collaboration, Search for flavour-changing neutral current top quark decays $t \rightarrow H q$ in $p p$ collisions at $\sqrt{s}=$ $8 \mathrm{TeV}$ with the ATLAS detector, J. High Energy Phys. 12 (2015) 061.

[18] ATLAS Collaboration, Search for top quark decays $t \rightarrow q H$ with $H \rightarrow \gamma \gamma$ using the ATLAS detector, J. High Energy Phys. 06 (2014) 008.

[19] CMS Collaboration, Search for top quark decays via Higgsboson-mediated flavor-changing neutral currents in $p p$ collisions at $\sqrt{s}=8 \mathrm{TeV}$, J. High Energy Phys. 02 (2017) 079.

[20] ATLAS Collaboration, Search for top quark decays $t \rightarrow q H$, with $H \rightarrow \gamma \gamma$, in $\sqrt{s}=13 \mathrm{TeV} p p$ collisions using the ATLAS detector, J. High Energy Phys. 10 (2017) 129.

[21] CMS Collaboration, Search for the flavor-changing neutral current interactions of the top quark and the Higgs boson which decays into a pair of $\mathrm{b}$ quarks at $\sqrt{s}=13 \mathrm{TeV}$, arXiv:1712.02399.
[22] ATLAS Collaboration, Evidence for the associated production of the Higgs boson and a top quark pair with the ATLAS detector, Phys. Rev. D 97, 072003 (2018).

[23] ATLAS Collaboration, The ATLAS Experiment at the CERN Large Hadron Collider, J. Instrum. 3, S08003 (2008).

[24] ATLAS Collaboration, ATLAS insertable B-layer technical design report, Report No. ATLAS-TDR-19, 2010, https:// cds.cern.ch/record/1291633; ATLAS insertable B-layer technical design report addendum, Report No. ATLASTDR-19-ADD-1, 2012, https://cds.cern.ch/record/1451888.

[25] M. Czakon and A. Mitov, Top++: A program for the calculation of the top-pair cross-section at hadron colliders, Comput. Phys. Commun. 185, 2930 (2014).

[26] ATLAS Collaboration, Performance of the ATLAS trigger system in 2015, Eur. Phys. J. C 77, 317 (2017).

[27] ATLAS Collaboration, Muon reconstruction performance of the ATLAS detector in proton-proton collision data at $\sqrt{s}=13 \mathrm{TeV}$, Eur. Phys. J. C 76, 292 (2016).

[28] ATLAS Collaboration, Electron efficiency measurements with the ATLAS detector using 2012 LHC proton-proton collision data, Eur. Phys. J. C 77, 195 (2017).

[29] ATLAS Collaboration, Topological cell clustering in the ATLAS calorimeters and its performance in LHC Run 1, Eur. Phys. J. C 77, 490 (2017).

[30] ATLAS Collaboration, Properties of jets and inputs to jet reconstruction and calibration with the ATLAS detector using proton-proton collisions at $\sqrt{s}=13 \mathrm{TeV}$, Report No. ATL-PHYS-PUB-2015-036, 2015, https://cds.cern.ch/ record/2044564.

[31] ATLAS Collaboration, Jet energy scale measurements and their systematic uncertainties in proton-proton collisions at $\sqrt{s}=13 \mathrm{TeV}$ with the ATLAS detector, Phys. Rev. D 96, 072002 (2017).

[32] M. Cacciari, G. P. Salam, and G. Soyez, The anti- $k_{t}$ jet clustering algorithm, J. High Energy Phys. 04 (2008) 063.

[33] M. Cacciari, G. P. Salam, and G. Soyez, FastJet user manual, Eur. Phys. J. C 72, 1896 (2012).

[34] ATLAS Collaboration, Performance of pile-up mitigation techniques for jets in $p p$ collisions at $\sqrt{s}=8 \mathrm{TeV}$ using the ATLAS detector, Eur. Phys. J. C 76, 581 (2016).

[35] ATLAS Collaboration, Performance of $b$-jet identification in the ATLAS experiment, J. Instrum. 11, P04008 (2016).

[36] ATLAS Collaboration, Optimisation of the ATLAS btagging performance for the 2016 LHC Run, Report No. ATL-PHYS-PUB-2016-012, 2016, https://cds.cern.ch/ record/2160731.

[37] ATLAS Collaboration, Reconstruction, Energy Calibration, and Identification of Hadronically Decaying Tau Leptons in the ATLAS Experiment for Run-2 of the LHC, Report No. ATL-PHYS-PUB-2015-045, 2015, https: //atlas.web .cern.ch/Atlas/GROUPS/PHYSICS/PUBNOTES/ATLPHYS-PUB-2015-045.

[38] ATLAS Collaboration, Measurement of the tau lepton reconstruction and identification performance in the ATLAS experiment using $p p$ collisions at $\sqrt{s}=13 \mathrm{TeV}$, Report No. ATLAS-CONF-2017-029, 2017, https://cds.cern.ch/ record/2261772.

[39] ATLAS Collaboration, Performance of missing transverse momentum reconstruction with the ATLAS detector 
using proton-proton collisions at $\sqrt{s}=13 \mathrm{TeV}$, arXiv: 1802.08168 .

[40] J. Alwall et al., The automated computation of tree-level and next-to-leading order differential cross sections, and their matching to parton shower simulations, J. High Energy Phys. 07 (2014) 079.

[41] P. Artoisenet, R. Frederix, O. Mattelaer, and R. Rietkerk, Automatic spin-entangled decays of heavy resonances in Monte Carlo simulations, J. High Energy Phys. 03 (2013) 015.

[42] T. Sjöstrand, S. Mrenna, and P. Z. Skands, A brief introduction to PYTHIA 8.1, Comput. Phys. Commun. 178, 852 (2008).

[43] M. Botje et al., The PDF4LHC Working Group interim recommendations, arXiv:1101.0538.

[44] A. D. Martin, W. J. Stirling, R. S. Thorne, and G. Watt, Uncertainties on $\alpha_{S}$ in global PDF analyses and implications for predicted hadronic cross sections, Eur. Phys. J. C 64, 653 (2009).

[45] J. Gao, M. Guzzi, J. Huston, H.-L. Lai, Z. Li, P. Nadolsky, J. Pumplin, D. Stump, and C.-P. Yuan, CT10 next-to-next-toleading order global analysis of QCD, Phys. Rev. D 89, 033009 (2014).

[46] R. D. Ball et al., Parton distributions with LHC data, Nucl. Phys. B867, 244 (2013).

[47] D. de Florian et al., Handbook of LHC Higgs cross sections: 4. Deciphering the nature of the Higgs sector, arXiv: 1610.07922.

[48] R. D. Ball et al., Parton distributions for the LHC Run II, J. High Energy Phys. 04 (2015) 040.

[49] ATLAS Collaboration, ATLAS Run 1 Pythia8 tunes, Repor No. ATL-PHYS-PUB-2014-021, 2014, https://cds.cern.ch/ record/1966419.

[50] T. Gleisberg, S. Höche, F. Krauss, M. Schönherr, S. Schumann, F. Siegert, and J. Winter, Event generation with SHERPA 1.1, J. High Energy Phys. 02 (2009) 007.
[51] S. Höche, F. Krauss, M. Schönherr, and F. Siegert, QCD matrix elements + parton showers. The NLO case, J. High Energy Phys. 04 (2013) 027.

[52] H.-L. Lai, M. Guzzi, J. Huston, Z. Li, P. M. Nadolsky, J. Pumplin, and C.-P. Yuan, New parton distributions for collider physics, Phys. Rev. D 82, 074024 (2010).

[53] S. Agostinelli et al., Geant4-a simulation toolkit, Nucl. Instrum. Methods Phys. Res., Sect. A 506, 250 (2003).

[54] ATLAS Collaboration, The ATLAS Simulation Infrastructure, Eur. Phys. J. C 70, 823 (2010).

[55] ATLAS Collaboration, The simulation principle and performance of the ATLAS fast calorimeter simulation FastCaloSim, Repot No. ATL-PHYS-PUB-2010-013, 2010, https://cds.cern.ch/record/1300517.

[56] DØ Collaboration, Extraction of the width of the $W$ boson from measurements of $\sigma(p \bar{p} \rightarrow W+X) \times B(W \rightarrow e \nu)$ and $\sigma(p \bar{p} \rightarrow Z+X) \times B(Z \rightarrow e e)$ and their ratio, Phys. Rev. D 61, 072001 (2000).

[57] ATLAS Collaboration, Estimation of non-prompt and fake lepton backgrounds in final states with top quarks produced in proton-proton collisions at $\sqrt{s}=8 \mathrm{TeV}$ with the ATLAS Detector, Repot No. ATLAS-CONF-2014-058, 2014, https://cds.cern.ch/record/1951336.

[58] A. Kalogeropoulos and J. Alwall, The SysCalc code: A tool to derive theoretical systematic uncertainties, arXiv: 1801.08401.

[59] ATLAS Collaboration, Luminosity determination in $p p$ collisions at $\sqrt{s}=8 \mathrm{TeV}$ using the ATLAS detector at the LHC, Eur. Phys. J. C 76, 653 (2016).

[60] A. L. Read, Presentation of search results: The $\mathrm{CL}_{\mathrm{s}}$ technique, J. Phys. G 28, 2693 (2002).

[61] ATLAS Collaboration, ATLAS computing acknowledgements, Repot No. ATL-GEN-PUB-2016-002, https://cds .cern.ch/record/2202407.

M. Aaboud ${ }^{34 d}$ G. Aad,${ }^{99}$ B. Abbott, ${ }^{124}$ O. Abdinov, ${ }^{13, \dagger}$ B. Abeloos, ${ }^{128}$ D. K. Abhayasinghe, ${ }^{91}$ S. H. Abidi, ${ }^{164}$ O. S. Abouzeid, ${ }^{143}$ N. L. Abraham, ${ }^{153}$ H. Abramowicz, ${ }^{158}$ H. Abreu, ${ }^{157}$ Y. Abulaiti, ${ }^{6}$ B. S. Acharya, ${ }^{67,67 b, m}$ S. Adachi, ${ }^{160}$ L. Adamczyk ${ }^{41 \mathrm{a}}$ J. Adelman, ${ }^{119}$ M. Adersberger, ${ }^{112}$ A. Adiguzel, ${ }^{12 \mathrm{c}}$ T. Adye, ${ }^{140}$ A. A. Affolder, ${ }^{143}$ Y. Afik, ${ }^{157}$ C. Agheorghiesei, ${ }^{27 \mathrm{c}}$ J. A. Aguilar-Saavedra, ${ }^{135 f, 135 a}$ F. Ahmadov, ${ }^{80, a i}$ G. Aielli, ${ }^{74 a}{ }^{74 b}$ S. Akatsuka, ${ }^{83}$ T. P. A. Åkesson, ${ }^{95}$ E. Akilli, ${ }^{55}$ A. V. Akimov, ${ }^{108}$ G. L. Alberghi, ${ }^{23 b, 23 a}$ J. Albert, ${ }^{173}$ P. Albicocco,${ }^{52}$ M. J. Alconada Verzini, ${ }^{86}$ S. Alderweireldt, ${ }^{117}$ M. Aleksa, ${ }^{35}$ I. N. Aleksandrov, ${ }^{80}$ C. Alexa, ${ }^{27 b}$ T. Alexopoulos,${ }^{10}$ M. Alhroob, ${ }^{124}$ B. Ali, ${ }^{137}$ M. Aliev, ${ }^{68 a, 68 b}$ G. Alimonti, ${ }^{69 a}$ J. Alison, ${ }^{36}$ S. P. Alkire, ${ }^{145}$ C. Allaire, ${ }^{128}$ B. M. M. Allbrooke, ${ }^{153}$ B. W. Allen, ${ }^{127}$ P. P. Allport, ${ }^{21}$ A. Aloisio, ${ }^{70 a, 70 b}$ A. Alonso, ${ }^{39}$ F. Alonso, ${ }^{86}$ C. Alpigiani, ${ }^{145}$ A. A. Alshehri, ${ }^{58}$ M. I. Alstaty, ${ }^{99}$ B. Alvarez Gonzalez, ${ }^{35}$ D. Álvarez Piqueras, ${ }^{171}$ M. G. Alviggi, ${ }^{70 a}{ }^{70 b}$ B. T. Amadio, ${ }^{18}$ Y. Amaral Coutinho, ${ }^{141 a}$ L. Ambroz, ${ }^{131}$ C. Amelung, ${ }^{26}$ D. Amidei, ${ }^{103}$ S. P. Amor Dos Santos, ${ }^{135 a, 135 c}$ S. Amoroso,${ }^{35}$ C. S. Amrouche, ${ }^{55}$ C. Anastopoulos, ${ }^{146}$ L. S. Ancu, ${ }^{55}$ N. Andari, ${ }^{21}$ T. Andeen, ${ }^{11}$ C. F. Anders, ${ }^{62 b}$ J. K. Anders,${ }^{20}$ K. J. Anderson, ${ }^{36}$ A. Andreazza, ${ }^{69 a, 69 b}$ V. Andrei, ${ }^{62 a}$ C. R. Anelli, ${ }^{173}$ S. Angelidakis,${ }^{37}$ I. Angelozzi,${ }^{18}$ A. Angerami, ${ }^{38}$ A. V. Anisenkov, ${ }^{120 b, 120 a}$ A. Annovi, ${ }^{72 a}$ C. Antel, ${ }^{62 a}$ M. T. Anthony, ${ }^{146}$ M. Antonelli, ${ }^{52}$ D. J. A. Antrim, ${ }^{168}$ F. Anulli, ${ }^{73 a}$ M. Aoki,${ }^{81}$ L. Aperio Bella, ${ }^{35}$ G. Arabidze,${ }^{104}$ Y. Arai, ${ }^{81}$ J. P. Araque, ${ }^{135 a}$ V. Araujo Ferraz, ${ }^{141 a}$ R. Araujo Pereira, ${ }^{141 a}$ A. T. H. Arce, ${ }^{49}$ R. E. Ardell, ${ }^{91}$ F. A. Arduh ${ }^{86}$ J-F. Arguin, ${ }^{107}$ S. Argyropoulos, ${ }^{78}$ A. J. Armbruster, ${ }^{35}$ L. J. Armitage, ${ }^{90}$ A. Armstrong, ${ }^{168}$ O. Arnaez, ${ }^{164}$ H. Arnold, ${ }^{118}$ M. Arratia, ${ }^{31}$ O. Arslan, ${ }^{24}$ A. Artamonov, ${ }^{109, \dagger}$ G. Artoni, ${ }^{131}$ S. Artz, ${ }^{97}$ S. Asai, ${ }^{160}$ N. Asbah, ${ }^{46}$ A. Ashkenazi, ${ }^{158}$ E. M. Asimakopoulou, ${ }^{169}$ L. Asquith, ${ }^{153}$ K. Assamagan, ${ }^{29}$ R. Astalos, ${ }^{28 a}$ R. J. Atkin, ${ }^{32 a}$ M. Atkinson, ${ }^{170}$ N. B. Atlay, ${ }^{148}$ K. Augsten, ${ }^{137}$ G. Avolio, ${ }^{35}$ 
R. Avramidou, ${ }^{61 a}$ B. Axen, ${ }^{18}$ M. K. Ayoub, ${ }^{15 a}$ G. Azuelos, ${ }^{107, a u}$ A. E. Baas, ${ }^{62 a}$ M. J. Baca, ${ }^{21}$ H. Bachacou, ${ }^{142}$ K. Bachas, ${ }^{68 a, 68 b}$ M. Backes, ${ }^{131}$ P. Bagnaia, ${ }^{73 a, 73 b}$ M. Bahmani, ${ }^{42}$ H. Bahrasemani, ${ }^{149}$ A. J. Bailey, ${ }^{171}$ J. T. Baines, ${ }^{140}$ M. Bajic ${ }^{39}$ C. Bakalis, ${ }^{10}$ O. K. Baker, ${ }^{180}$ P. J. Bakker, ${ }^{118}$ D. Bakshi Gupta, ${ }^{93}$ E. M. Baldin, ${ }^{120 b, 120 a}$ P. Balek, ${ }^{177}$ F. Balli, ${ }^{142}$ W. K. Balunas, ${ }^{132}$ E. Banas, ${ }^{42}$ A. Bandyopadhyay, ${ }^{24}$ Sw. Banerjee, ${ }^{178, i}$ A. A. E. Bannoura, ${ }^{179}$ L. Barak, ${ }^{158}$ W. M. Barbe, ${ }^{37}$ E. L. Barberio, ${ }^{102}$ D. Barberis, ${ }^{56 b, 56 a}$ M. Barbero, ${ }^{99}$ T. Barillari, ${ }^{113}$ M-S Barisits, ${ }^{35}$ J. Barkeloo, ${ }^{127}$ T. Barklow, ${ }^{150}$ N. Barlow, ${ }^{31}$ R. Barnea, ${ }^{157}$ S. L. Barnes, ${ }^{61 c}$ B. M. Barnett, ${ }^{140}$ R. M. Barnett, ${ }^{18}$ Z. Barnovska-Blenessy, ${ }^{61 a}$ A. Baroncelli, ${ }^{75 a}$ G. Barone, ${ }^{26}$ A. J. Barr, ${ }^{131}$ L. Barranco Navarro, ${ }^{171}$ F. Barreiro, ${ }^{96}$ J. Barreiro Guimar aes da Costa, ${ }^{15 a}$ R. Bartoldus, ${ }^{150}$ A. E. Barton, ${ }^{87}$ P. Bartos, ${ }^{28 a}$ A. Basalaev, ${ }^{133}$ A. Bassalat, ${ }^{128}$ R. L. Bates, ${ }^{58}$ S. J. Batista, ${ }^{164}$ S. Batlamous, ${ }^{34 e}$ J. R. Batley, ${ }^{31}$ M. Battaglia, ${ }^{143}$

M. Bauce, ${ }^{73 a, 73 b}$ F. Bauer, ${ }^{142}$ K. T. Bauer, ${ }^{168}$ H. S. Bawa, ${ }^{150, k}$ J. B. Beacham, ${ }^{122}$ M. D. Beattie, ${ }^{87}$ T. Beau, ${ }^{94}$

P. H. Beauchemin, ${ }^{167}$ P. Bechtle, ${ }^{24}$ H. C. Beck, ${ }^{54}$ H. P. Beck, ${ }^{20, r}$ K. Becker,${ }^{53}$ M. Becker, ${ }^{97}$ C. Becot, ${ }^{46}$ A. Beddall, ${ }^{12 d}$ A. J. Beddall, ${ }^{12 \mathrm{a}}$ V. A. Bednyakov, ${ }^{80}$ M. Bedognetti, ${ }^{118}$ C. P. Bee, ${ }^{152}$ T. A. Beermann, ${ }^{35}$ M. Begalli, ${ }^{141 \mathrm{a}}$ M. Begel, ${ }^{29}$ A. Behera, ${ }^{152}$ J. K. Behr, ${ }^{46}$ A. S. Bell, ${ }^{92}$ G. Bella, ${ }^{158}$ L. Bellagamba, ${ }^{23 b}$ A. Bellerive, ${ }^{33}$ M. Bellomo, ${ }^{157}$ P. Bellos, ${ }^{9}$ K. Belotskiy, ${ }^{110}$ N. L. Belyaev, ${ }^{110}$ O. Benary, ${ }^{158, \dagger}$ D. Benchekroun, ${ }^{34 a}$ M. Bender, ${ }^{112}$ N. Benekos, ${ }^{10}$ Y. Benhammou, ${ }^{158}$ E. Benhar Noccioli, ${ }^{180}$ J. Benitez, ${ }^{78}$ D. P. Benjamin, ${ }^{49}$ M. Benoit, ${ }^{55}$ J. R. Bensinger, ${ }^{26}$ S. Bentvelsen, ${ }^{118}$ L. Beresford, ${ }^{131}$ M. Beretta, ${ }^{52}$ D. Berge, ${ }^{46}$ E. Bergeaas Kuutmann, ${ }^{169}$ N. Berger, ${ }^{5}$ L. J. Bergsten, ${ }^{26}$ J. Beringer, $^{18}$ S. Berlendis, ${ }^{7}$ N. R. Bernard, ${ }^{100}$ G. Bernardi, ${ }^{94}$ C. Bernius, ${ }^{150}$ F. U. Bernlochner, ${ }^{24}$ T. Berry, ${ }^{91}$ P. Berta, ${ }^{97}$ C. Bertella, ${ }^{15 a}$ G. Bertoli, ${ }^{45,45 b}$ I. A. Bertram, ${ }^{87}$ G. J. Besjes, ${ }^{39}$ O. Bessidskaia Bylund, ${ }^{45 a, 45 b}$ M. Bessner, ${ }^{46}$ N. Besson, ${ }^{142}$ A. Bethani, ${ }^{98}$ S. Bethke, ${ }^{113}$ A. Betti, ${ }^{24}$ A. J. Bevan, ${ }^{90}$ J. Beyer, ${ }^{113}$ R. M. Bianchi, ${ }^{134}$ O. Biebel, ${ }^{112}$ D. Biedermann, ${ }^{19}$ R. Bielski, ${ }^{98}$ K. Bierwagen, ${ }^{97}$ N. V. Biesuz, ${ }^{72 a, 72 b}$ M. Biglietti, ${ }^{75 a}$ T. R. V. Billoud, ${ }^{107}$ M. Bindi, ${ }^{54}$ A. Bingul, ${ }^{12 d}$ C. Bini, ${ }^{73 a, 73 b}$ S. Biondi, ${ }^{23 b, 23 a}$ T. Bisanz, ${ }^{54}$ J. P. Biswal, ${ }^{158}$ C. Bittrich, ${ }^{48}$ D. M. Bjergaard, ${ }^{49}$ J. E. Black, ${ }^{150}$ K. M. Black, ${ }^{25}$ R. E. Blair, ${ }^{6}$ T. Blazek, ${ }^{28 a}$ I. Bloch, $^{46}$ C. Blocker, ${ }^{26}$ A. Blue, ${ }^{58}$ U. Blumenschein, ${ }^{90}$ Dr. Blunier, ${ }^{144 a}$ G. J. Bobbink, ${ }^{118}$ V. S. Bobrovnikov, ${ }^{120 b, 120 a}$ S. S. Bocchetta, ${ }^{95}$ A. Bocci, ${ }^{49}$ D. Boerner, ${ }^{179}$ D. Bogavac, ${ }^{112}$ A. G. Bogdanchikov, ${ }^{120 b, 120 a}$ C. Bohm, ${ }^{45 a}$ V. Boisvert, ${ }^{91}$ P. Bokan, ${ }^{169, \text { aa }}$ T. Bold, ${ }^{41 a}$ A. S. Boldyrev, ${ }^{111}$ A. E. Bolz, ${ }^{62 b}$ M. Bomben, ${ }^{94}$ M. Bona, ${ }^{90}$ J. S. B. Bonilla, ${ }^{127}$ M. Boonekamp, ${ }^{142}$ A. Borisov, ${ }^{139}$ G. Borissov, ${ }^{87}$ J. Bortfeldt, ${ }^{35}$ D. Bortoletto, ${ }^{131}$ V. Bortolotto, ${ }^{74 a, 74 b}$ D. Boscherini, ${ }^{23 b}$ M. Bosman, ${ }^{14}$ J. D. Bossio Sola, ${ }^{30}$ K. Bouaouda, ${ }^{34 a}$ J. Boudreau, ${ }^{134}$ E. V. Bouhova-Thacker, ${ }^{87}$ D. Boumediene, ${ }^{37}$ C. Bourdarios, ${ }^{128}$ S. K. Boutle, ${ }^{58}$

A. Boveia, ${ }^{122}$ J. Boyd, ${ }^{35}$ I. R. Boyko, ${ }^{80}$ A. J. Bozson, ${ }^{91}$ J. Bracinik, ${ }^{21}$ N. Brahimi, ${ }^{99}$ A. Brandt, ${ }^{8}$ G. Brandt, ${ }^{179}$ O. Brandt, ${ }^{62 a}$ F. Braren, ${ }^{46}$ U. Bratzler, ${ }^{161}$ B. Brau, ${ }^{100}$ J. E. Brau, ${ }^{127}$ W. D. Breaden Madden, ${ }^{58}$ K. Brendlinger, ${ }^{46}$ A. J. Brennan, ${ }^{102}$ L. Brenner, ${ }^{46}$ R. Brenner, ${ }^{169}$ S. Bressler, ${ }^{177}$ B. Brickwedde, ${ }^{97}$ D. L. Briglin, ${ }^{21}$ D. Britton, ${ }^{58}$ D. Britzger, ${ }^{62 b}$ I. Brock, $^{24}$ R. Brock, ${ }^{104}$ G. Brooijmans, ${ }^{38}$ T. Brooks, ${ }^{91}$ W. K. Brooks, ${ }^{144 b}$ E. Brost, ${ }^{119}$ J. H Broughton, ${ }^{21}$ P. A. Bruckman de Renstrom, ${ }^{42}$ D. Bruncko, ${ }^{28 b}$ A. Bruni, ${ }^{23 b}$ G. Bruni, ${ }^{23 b}$ L. S. Bruni, ${ }^{118}$ S. Bruno, ${ }^{74 a, 74 b}$ B. H. Brunt, ${ }^{31}$ M. Bruschi, ${ }^{23 b}$ N. Bruscino, ${ }^{134}$ P. Bryant, ${ }^{36}$ L. Bryngemark, ${ }^{46}$ T. Buanes, ${ }^{17}$ Q. Buat, ${ }^{35}$ P. Buchholz, ${ }^{148}$ A. G. Buckley, ${ }^{58}$ I. A. Budagov, ${ }^{80}$ F. Buehrer, ${ }^{53}$ M. K. Bugge, ${ }^{130}$ O. Bulekov, ${ }^{110}$ D. Bullock, ${ }^{8}$ T. J. Burch, ${ }^{119}$ S. Burdin, ${ }^{88}$ C. D. Burgard, ${ }^{118}$ A. M. Burger, ${ }^{5}$ B. Burghgrave, ${ }^{119}$ K. Burka, ${ }^{42}$ S. Burke, ${ }^{140}$ I. Burmeister, ${ }^{47}$ J. T. P. Burr, ${ }^{131}$ D. Büscher, ${ }^{53}$ V. Büscher, ${ }^{97}$ E. Buschmann, ${ }^{54}$ P. Bussey, ${ }^{58}$ J. M. Butler, ${ }^{25}$ C. M. Buttar, ${ }^{58}$ J. M. Butterworth, ${ }^{92}$ P. Butti, ${ }^{35}$ W. Buttinger, ${ }^{35}$ A. Buzatu, ${ }^{155}$ A. R. Buzykaev, ${ }^{120 b, 120 a}$ G. Cabras, ${ }^{23 b, 23 a}$ S. Cabrera Urbán, ${ }^{171}$ D. Caforio, ${ }^{137}$ H. Cai, ${ }^{170}$ V. M. M. Cairo, ${ }^{2}$ O. Cakir, ${ }^{4 a}$ N. Calace, ${ }^{55}$ P. Calafiura, ${ }^{18}$ A. Calandri, ${ }^{99}$ G. Calderini, ${ }^{94}$ P. Calfayan, ${ }^{66}$ G. Callea, ${ }^{40 b, 40 a}$ L. P. Caloba, ${ }^{141 a}$ S. Calvente Lopez, ${ }^{96}$ D. Calvet, ${ }^{37}$ S. Calvet, ${ }^{37}$ T. P. Calvet, ${ }^{152}$ M. Calvetti, ${ }^{72 a, 72 b}$ R. Camacho Toro, ${ }^{94}$ S. Camarda, ${ }^{35}$ P. Camarri, ${ }^{74 a, 74 b}$ D. Cameron, ${ }^{130}$

R. Caminal Armadans, ${ }^{100}$ C. Camincher, ${ }^{35}$ S. Campana, ${ }^{35}$ M. Campanelli, ${ }^{92}$ A. Camplani, ${ }^{39}$ A. Campoverde, ${ }^{148}$ V. Canale, ${ }^{70 a, 70 b}$ M. Cano Bret, ${ }^{61 \mathrm{c}}$ J. Cantero, ${ }^{125}$ T. Cao, ${ }^{158}$ Y. Cao, ${ }^{170}$ M. D. M. Capeans Garrido, ${ }^{35}$ I. Caprini, ${ }^{27 b}$ M. Caprini, ${ }^{27 b}$ M. Capua, ${ }^{40 b, 40 a}$ R. M. Carbone, ${ }^{38}$ R. Cardarelli, ${ }^{74 a}$ F. Cardillo, ${ }^{53}$ I. Carli, ${ }^{138}$ T. Carli, ${ }^{35}$ G. Carlino, ${ }^{70 a}$ B. T. Carlson, ${ }^{134}$ L. Carminati, ${ }^{69 a, 69 b}$ R. M. D. Carney, ${ }^{45 a, 45 b}$ S. Caron, ${ }^{117}$ E. Carquin, ${ }^{144 b}$ S. Carrá, ${ }^{69 a, 69 b}$ G. D. Carrillo-Montoya, ${ }^{35}$ D. Casadei, ${ }^{32 b}$ M. P. Casado, ${ }^{14, e}$ A. F. Casha, ${ }^{164}$ M. Casolino, ${ }^{14}$ D. W. Casper, ${ }^{168}$ R. Castelijn, ${ }^{118}$ F. L. Castillo, ${ }^{171}$ V. Castillo Gimenez, ${ }^{171}$ N. F. Castro, ${ }^{135 a, 135 e}$ A. Catinaccio, ${ }^{35}$ J. R. Catmore, ${ }^{130}$ A. Cattai, ${ }^{35}$ J. Caudron, ${ }^{24}$ V. Cavaliere, ${ }^{29}$ E. Cavallaro, ${ }^{14}$ D. Cavalli, ${ }^{69 a}$ M. Cavalli-Sforza, ${ }^{14}$ V. Cavasinni, ${ }^{72 a, 72 b}$ E. Celebi, ${ }^{12 b}$ F. Ceradini, ${ }^{75 a, 75 b}$ L. Cerda Alberich, ${ }^{171}$ A. S. Cerqueira, ${ }^{141 b}$ A. Cerri, ${ }^{153}$ L. Cerrito, ${ }^{74 a, 74 b}$ F. Cerutti, ${ }^{18}$ A. Cervelli, ${ }^{23 b, 23 a}$ S. A. Cetin, ${ }^{12 b}$ A. Chafaq, ${ }^{34 a}$ DC Chakraborty, ${ }^{119}$ S. K. Chan, ${ }^{60}$ W. S. Chan, ${ }^{118}$ Y. L. Chan, ${ }^{64 a}$ J. D. Chapman, ${ }^{31}$ D. G. Charlton, ${ }^{21}$ C. C. Chau, ${ }^{33}$ C. A. Chavez Barajas, ${ }^{153}$ S. Che, ${ }^{122}$ A. Chegwidden, ${ }^{104}$ S. Chekanov, ${ }^{6}$ S. V. Chekulaev, ${ }^{165 a}$ G. A. Chelkov, ${ }^{80, a t}$ M. A. Chelstowska, ${ }^{35}$ C. Chen, ${ }^{61 a}$ C. Chen, ${ }^{79}$ H. Chen, ${ }^{29}$ J. Chen, ${ }^{61 a}$ J. Chen, ${ }^{38}$ S. Chen, ${ }^{15 b}$ S. Chen, ${ }^{132}$ X. Chen, ${ }^{15 c, a s}$ Y. Chen, ${ }^{82}$ Y.-H. Chen, ${ }^{46}$ H. C. Cheng, ${ }^{103}$ H. J. Cheng, ${ }^{15 d}$ A. Cheplakov, ${ }^{80}$ E. Cheremushkina, ${ }^{139}$ R. Cherkaoui El Moursli, ${ }^{34}$ 
E. Cheu, ${ }^{7}$ K. Cheung, ${ }^{65}$ L. Chevalier ${ }^{142}$ V. Chiarella, ${ }^{52}$ G. Chiarelli, ${ }^{72 a}$ G. Chiodini, ${ }^{68 a}$ A. S. Chisholm, ${ }^{35}$ A. Chitan, ${ }^{27 b}$ I. Chiu, ${ }^{160}$ Y. H. Chiu, ${ }^{173}$ M. V. Chizhov, ${ }^{80}$ K. Choi, ${ }^{66}$ A. R. Chomont, ${ }^{128}$ S. Chouridou, ${ }^{159}$ Y. S. Chow, ${ }^{118}$ V. Christodoulou, ${ }^{92}$ M. C. Chu, ${ }^{64 a}$ J. Chudoba, ${ }^{136}$ A. J. Chuinard,${ }^{101}$ J. J. Chwastowski, ${ }^{42}$ L. Chytka, ${ }^{126}$ D. Cinca, ${ }^{47}$ V. Cindro, ${ }^{89}$ I. A. Cioară, ${ }^{24}$ A. Ciocio, ${ }^{18}$ F. Cirotto, ${ }^{70 a}, 70 \mathrm{~b}$ Z. H. Citron, ${ }^{177}$ M. Citterio, ${ }^{69 a}$ A. Clark, ${ }^{55}$ M. R. Clark ${ }^{38}$ P. J. Clark, ${ }^{50}$ C. Clement, ${ }^{45,45 b}$ Y. Coadou, ${ }^{99}$ M. Cobal, ${ }^{67 a, 67 \mathrm{c}}$ A. Coccaro, ${ }^{56 b, 56 \mathrm{a}}$ J. Cochran, ${ }^{79}$ A. E. C. Coimbra, ${ }^{177}$ L. Colasurdo, ${ }^{117}$ B. Cole, ${ }^{38}$ A. P. Colijn, ${ }^{118}$ J. Collot,${ }^{59}$ P. Conde Mui no, ${ }^{135 a, 135 b}$ E. Coniavitis,${ }^{53}$ S. H. Connell, ${ }^{32 b}$ I. A. Connelly, ${ }^{98}$ S. Constantinescu, ${ }^{27 b}$ F. Conventi, ${ }^{70 a, a v}$ A. M. Cooper-Sarkar, ${ }^{131}$ F. Cormier, ${ }^{172}$ K. J. R. Cormier, ${ }^{164}$ M. Corradi, ${ }^{73 a, 73 b}$ E. E. Corrigan, ${ }^{95}$ F. Corriveau, ${ }^{101, \text { ag }}$ A. Cortes-Gonzalez, ${ }^{35}$ M. J. Costa, ${ }^{171}$ D. Costanzo, ${ }^{146}$ G. Cottin, ${ }^{31}$ G. Cowan, ${ }^{91}$ B. E. Cox ${ }^{98}$ J. Crane, ${ }^{98}$ K. Cranmer, ${ }^{121}$ S. J. Crawley, ${ }^{58}$ R. A. Creager, ${ }^{132}$ G. Cree, ${ }^{33}$ S. Crépé-Renaudin, ${ }^{59}$ F. Crescioli, ${ }^{94}$ M. Cristinziani, ${ }^{24}$ V. Croft, ${ }^{121}$ G. Crosetti, ${ }^{40 b, 40 a}$ A. Cueto, ${ }^{96}$ T. Cuhadar Donszelmann, ${ }^{146}$ A. R. Cukierman, ${ }^{150}$ M. Curatolo, ${ }^{52}$ J. Cúth, ${ }^{97}$ S. Czekierda,${ }^{42}$ P. Czodrowski, ${ }^{35}$ M. J. Da Cunha Sargedas De Sousa, ${ }^{61 b, 135 b}$ C. Da Via, ${ }^{98}$ W. Dabrowski, ${ }^{41 \mathrm{a}}$ T. Dado, ${ }^{28 a, a a}$ S. Dahbi, ${ }^{34 \mathrm{e}}$ T. Dai, ${ }^{103}$ F. Dallaire, ${ }^{107}$ C. Dallapiccola, ${ }^{100}$ M. Dam, ${ }^{39}$ G. D'amen, ${ }^{23 b, 23 a}$ J. Damp, ${ }^{97}$ J. R. Dandoy, ${ }^{132}$ M. F. Daneri, ${ }^{30}$ N. P. Dang, ${ }^{178, i}$ N. D Dann, ${ }^{98}$ M. Danninger, ${ }^{172}$ V. Dao, ${ }^{35}$ G. Darbo, ${ }^{56 b}$ S. Darmora, ${ }^{8}$ O. Dartsi, ${ }^{5}$ A. Dattagupta, ${ }^{127}$ T. Daubney, ${ }^{46}$ S. D'Auria ${ }^{58}$ W. Davey,${ }^{24}$ C. David, ${ }^{46}$ T. Davidek, ${ }^{138}$ D. R. Davis, ${ }^{49}$ E. Dawe, ${ }^{102}$ I. Dawson, ${ }^{146}$ K. De,${ }^{8}$ R. de Asmundis, ${ }^{70 a}$ A. De Benedetti, ${ }^{124}$ S. De Castro, ${ }^{23 b, 23 a}$ S. De Cecco, ${ }^{73 a, 73 b}$ N. De Groot, ${ }^{117}$ P. de Jong, ${ }^{118}$ H. De la Torre, ${ }^{104}$ F. De Lorenzi, ${ }^{79}$ A. De Maria ${ }^{54, s}$ D. De Pedis, ${ }^{73 a}$ A. De Salvo, ${ }^{73 a}$ U. De Sanctis, ${ }^{74 a}, 74 b$ A. De Santo, ${ }^{153}$ K. De Vasconcelos Corga,${ }^{99}$ J. B. De Vivie De Regie, ${ }^{128}$ C. Debenedetti, ${ }^{143}$ D. V. Dedovich, ${ }^{80}$ N. Dehghanian, ${ }^{3}$ M. Del Gaudio, ${ }^{40 b, 40 a}$ J. Del Peso, ${ }^{96}$ D. Delgove,${ }^{128}$ F. Deliot,${ }^{142}$ C. M. Delitzsch, ${ }^{7}$ M. Della Pietra, ${ }^{70 a, 70 b}$ D. della Volpe,${ }^{55}$ A. Dell'Acqua, ${ }^{35}$ L. Dell'Asta, ${ }^{25}$ M. Delmastro, ${ }^{5}$ C. Delporte, ${ }^{128}$ P. A. Delsart, ${ }^{59}$ D. A. DeMarco, ${ }^{164}$ S. Demers ${ }^{180}$ M. Demichev ${ }^{80}$ S. P. Denisov, ${ }^{139}$ D. Denysiuk,${ }^{118}$ L. D'Eramo, ${ }^{94}$ D. Derendarz, ${ }^{42}$ J. E. Derkaoui, ${ }^{34 d}$ F. Derue, ${ }^{94}$ P. Dervan, ${ }^{88}$ K. Desch,${ }^{24}$ C. Deterre ${ }^{46}$ K. Dette, ${ }^{164}$ M. R. Devesa,${ }^{30}$ P. O. Deviveiros, ${ }^{35}$ A. Dewhurst, ${ }^{140}$ S. Dhaliwal, ${ }^{26}$ F. A. Di Bello, ${ }^{55}$ A. Di Ciaccio, ${ }^{74 a, 74 b}$ L. Di Ciaccio, ${ }^{5}$ W. K. Di Clemente, ${ }^{132}$ C. Di Donato, ${ }^{70 a, 70 b}$ A. Di Girolamo, ${ }^{35}$ B. Di Micco,${ }^{75 a, 75 b}$ R. Di Nardo, ${ }^{35}$ K. F. Di Petrillo, ${ }^{60}$ A. Di Simone, ${ }^{53}$ R. Di Sipio, ${ }^{164}$ D. Di Valentino, ${ }^{33}$ C. Diaconu, ${ }^{99}$ M. Diamond, ${ }^{164}$ F. A. Dias, ${ }^{39}$ T. Dias do Vale, ${ }^{135 a}$ M. A. Diaz, ${ }^{144 a}$ J. Dickinson, ${ }^{18}$ E. B. Diehl,${ }^{103}$ J. Dietrich, ${ }^{19}$ S. Díez Cornell ${ }^{46}$ A. Dimitrievska, ${ }^{18}$ J. Dingfelder, ${ }^{24}$ F. Dittus,${ }^{35}$ F. Djama, ${ }^{99}$ T. Djobava, ${ }^{156 \mathrm{~b}}$ J. I. Djuvsland, ${ }^{62 \mathrm{a}}$ M. A. B. do Vale, ${ }^{141 \mathrm{c}}$ M. Dobre, ${ }^{27 \mathrm{~b}}$ D. Dodsworth, ${ }^{26}$ C. Doglioni, ${ }^{95}$ J. Dolejsi, ${ }^{138}$ Z. Dolezal, ${ }^{138}$ M. Donadelli, ${ }^{141 \mathrm{~d}}$ J. Donini, ${ }^{37}$ A. D'onofrio, ${ }^{90}$ M. D’Onofrio, ${ }^{88}$ J. Dopke, ${ }^{140}$ A. Doria,${ }^{70 a}$ M. T. Dova, ${ }^{86}$ A. T. Doyle, ${ }^{58}$ E. Drechsler, ${ }^{54}$ E. Dreyer, ${ }^{149}$ T. Dreyer, ${ }^{54}$ M. Dris,${ }^{10}$ Y. Du, ${ }^{61 b}$ J. Duarte-Campderros, ${ }^{158}$ F. Dubinin, ${ }^{108}$ M. Dubovsky, ${ }^{28 a}$ A. Dubreuil,${ }^{55}$ E. Duchovni, ${ }^{177}$ G. Duckeck, ${ }^{112}$ A. Ducourthial, ${ }^{94}$ O. A. Ducu, ${ }^{107, z}$ D. Duda, ${ }^{113}$ A. Dudarev, ${ }^{35}$ A. Chr. Dudder, ${ }^{97}$ E. M. Duffield, ${ }^{18}$ L. Duflot,${ }^{128}$ M. Dührssen, ${ }^{35}$ C. Dülsen, ${ }^{179}$ M. Dumancic, ${ }^{177}$ A. E. Dumitriu, ${ }^{27 b, d}$ A. K. Duncan, ${ }^{58}$ M. Dunford, ${ }^{62 \mathrm{a}}$ A. Duperrin, ${ }^{99}$ H. Duran Yildiz, ${ }^{4 \mathrm{a}}$ M. Düren, ${ }^{57}$ A. Durglishvili, ${ }^{156 \mathrm{~b}}$ D. Duschinger, ${ }^{48}$ B. Dutta, ${ }^{46}$ D. Duvnjak, ${ }^{1}$ M. Dyndal, ${ }^{46}$ S. Dysch, ${ }^{98}$ B. S. Dziedzic, ${ }^{42}$ C. Eckardt, ${ }^{46}$ K. M. Ecker, ${ }^{113}$ R. C. Edgar,${ }^{103}$ T. Eifert, ${ }^{35}$ G. Eigen, ${ }^{17}$ K. Einsweiler, ${ }^{18}$ T. Ekelof, ${ }^{169}$ M. El Kacimi, ${ }^{34 \mathrm{c}}$ R. El Kosseifi, ${ }^{99}$ V. Ellajosyula,${ }^{99}$ M. Ellert, ${ }^{169}$ F. Ellinghaus, ${ }^{179}$ A. A. Elliot, ${ }^{90}$ N. Ellis, ${ }^{35}$ J. Elmsheuser, ${ }^{29}$ M. Elsing, ${ }^{35}$ D. Emeliyanov, ${ }^{140}$ Y. Enari, ${ }^{160}$ J. S. Ennis, ${ }^{175}$ M. B. Epland, ${ }^{49}$ J. Erdmann, ${ }^{47}$ A. Ereditato, ${ }^{20}$ S. Errede, ${ }^{170}$ M. Escalier, ${ }^{128}$ C. Escobar, ${ }^{171}$ B. Esposito,${ }^{52}$ O. Estrada Pastor, ${ }^{171}$ A. I. Etienvre, ${ }^{142}$ E. Etzion, ${ }^{158}$ H. Evans, ${ }^{66}$ A. Ezhilov, ${ }^{133}$ M. Ezzi, ${ }^{34 \mathrm{e}}$ F. Fabbri, ${ }^{23 b, 23 a}$ L. Fabbri, ${ }^{23 b, 23 a}$ V. Fabiani, ${ }^{117}$ G. Facini, ${ }^{92}$ R. M. Faisca Rodrigues Pereira, ${ }^{135 a}$ R. M. Fakhrutdinov, ${ }^{139}$ S. Falciano, ${ }^{73 a}$ P. J. Falke, ${ }^{5}$ S. Falke, ${ }^{5}$ J. Faltova, ${ }^{138}$ Y. Fang, ${ }^{15 a}$ M. Fanti, ${ }^{69,69 b}$ A. Farbin, ${ }^{8}$ A. Farilla, ${ }^{75 a}$ E. M. Farina, ${ }^{71 a, 71 b}$ T. Farooque,${ }^{104}$ S. Farrell, ${ }^{18}$ S. M. Farrington, ${ }^{175}$ P. Farthouat, ${ }^{35}$ F. Fassi, ${ }^{34 \mathrm{e}}$ P. Fassnacht, ${ }^{35}$ D. Fassouliotis, ${ }^{9}$ M. Faucci Giannelli, ${ }^{50}$ A. Favareto, ${ }^{56 \mathrm{~b}, 56 \mathrm{a}}$ W. J. Fawcett, ${ }^{55}$ L. Fayard, ${ }^{128}$ O. L. Fedin, ${ }^{133,0}$ W. Fedorko, ${ }^{172}$ M. Feickert, ${ }^{43}$ S. Feigl, ${ }^{130}$ L. Feligioni,${ }^{99}$ C. Feng, ${ }^{61 b}$ E. J. Feng, ${ }^{35}$ M. Feng, ${ }^{49}$ M. J. Fenton, ${ }^{58}$ A. B. Fenyuk, ${ }^{139}$ L. Feremenga, ${ }^{8}$ J. Ferrando, ${ }^{46}$ A. Ferrari, ${ }^{169}$ P. Ferrari, ${ }^{118}$ R. Ferrari, ${ }^{71 a}$ D. E. Ferreira de Lima, ${ }^{62 b}$ A. Ferrer ${ }^{171}$ D. Ferrere, ${ }^{55}$ C. Ferretti,${ }^{103}$ F. Fiedler, ${ }^{97}$ A. Filipčičč ${ }^{89}$ F. Filthaut, ${ }^{117}$ K. D. Finelli ${ }^{25}$ M. C. N. Fiolhais, ${ }^{135 a, 135 c, a}$ L. Fiorini,${ }^{171}$ C. Fischer,${ }^{14}$ W. C. Fisher,${ }^{104}$ N. Flaschel,${ }^{46}$ I. Fleck, ${ }^{148}$ P. Fleischmann, ${ }^{103}$ R. R. M. Fletcher, ${ }^{132}$ T. Flick, ${ }^{179}$ B. M. Flierl, ${ }^{112}$ L. M. Flores, ${ }^{132}$ L. R. Flores Castillo, ${ }^{64 a}$ N. Fomin,${ }^{17}$ G. T. Forcolin, ${ }^{98}$ A. Formica, ${ }^{142}$ F. A. Förster, ${ }^{14}$ A. C. Forti, ${ }^{98}$ A. G. Foster, ${ }^{21}$ D. Fournier, ${ }^{128}$ H. Fox, ${ }^{87}$ S. Fracchia, ${ }^{146}$ P. Francavilla, ${ }^{72 a, 72 b}$ M. Franchini, ${ }^{23 b, 23 a}$ S. Franchino, ${ }^{62 a}$ D. Francis,${ }^{35}$ L. Franconi, ${ }^{130}$ M. Franklin, ${ }^{60}$ M. Frate, ${ }^{168}$ M. Fraternali, ${ }^{71,71 b}$ D. Freeborn, ${ }^{92}$ S. M. Fressard-Batraneanu, ${ }^{35}$ B. Freund, ${ }^{107}$ W. S. Freund,${ }^{141 a}$ D. Froidevaux, ${ }^{35}$ J. A. Frost ${ }^{131}$ C. Fukunaga, ${ }^{161}$ T. Fusayasu, ${ }^{114}$ J. Fuster, ${ }^{171}$ O. Gabizon, ${ }^{157}$ A. Gabrielli, ${ }^{23 b, 23 a}$ A. Gabrielli, ${ }^{18}$ G. P. Gach, ${ }^{41 a}$ S. Gadatsch, ${ }^{55}$ P. Gadow, ${ }^{113}$ G. Gagliardi, ${ }^{56 b, 56 a}$ L. G. Gagnon, ${ }^{107}$ C. Galea, ${ }^{27 b}$ B. Galhardo, ${ }^{135 a, 135 c}$ E. J. Gallas, ${ }^{131}$ 
B. J. Gallop, ${ }^{140}$ P. Gallus, ${ }^{137}$ G. Galster, ${ }^{39}$ R. Gamboa Goni,${ }^{90}$ K. K. Gan, ${ }^{122}$ S. Ganguly, ${ }^{177}$ Y. Gao, ${ }^{88}$ Y. S. Gao, ${ }^{150, k}$ C. García, ${ }^{171}$ J. E. García Navarro, ${ }^{171}$ J. A. García Pascual, ${ }^{15 a}$ M. Garcia-Sciveres, ${ }^{18}$ R. W. Gardner, ${ }^{36}$ N. Garelli, ${ }^{150}$ V. Garonne, ${ }^{130}$ K. Gasnikova, ${ }^{46}$ A. Gaudiello,${ }^{56,56 a}$ G. Gaudio, ${ }^{71 a}$ I. L. Gavrilenko, ${ }^{108}$ A. Gavrilyuk, ${ }^{109}$ C. Gay, ${ }^{172}$ G. Gaycken, ${ }^{24}$ E. N. Gazis, ${ }^{10}$ C. N. P. Gee, ${ }^{140}$ J. Geisen, ${ }^{54}$ M. Geisen, ${ }^{97}$ M. P. Geisler, ${ }^{62 \mathrm{a}}$ K. Gellerstedt, ${ }^{45,45 \mathrm{~b}}$ C. Gemme,${ }^{56 \mathrm{~b}}$ M. H. Genest, ${ }^{59}$ C. Geng, ${ }^{103}$ S. Gentile, ${ }^{73 a, 73 b}$ C. Gentsos, ${ }^{159}$ S. George, ${ }^{91}$ D. Gerbaudo, ${ }^{14}$ G. Gessner, ${ }^{47}$ S. Ghasemi ${ }^{148}$ M. Ghasemi Bostanabad, ${ }^{173}$ M. Ghneimat, ${ }^{24}$ B. Giacobbe, ${ }^{23 b}$ S. Giagu, ${ }^{73 a, 73 b}$ N. Giangiacomi, ${ }^{23 b, 23 a}$ P. Giannetti, ${ }^{72 a}$ S. M. Gibson, ${ }^{91}$ M. Gignac, ${ }^{143}$ D. Gillberg, ${ }^{33}$ G. Gilles, ${ }^{179}$ D. M. Gingrich,,${ }^{3, a u}$ M. P. Giordani, ${ }^{67 a, 67 c}$ F. M. Giorgi, ${ }^{23 b}$ P. F. Giraud ${ }^{142}$ P. Giromini, ${ }^{60}$ G. Giugliarelli, ${ }^{67 a, 67 c}$ D. Giugni, ${ }^{69 a}$ F. Giuli, ${ }^{131}$ M. Giulini, ${ }^{6 b}$ S. Gkaitatzis, ${ }^{159}$ I. Gkialas, ${ }^{9, h}$ E. L. Gkougkousis, ${ }^{14}$ P. Gkountoumis, ${ }^{10}$ L. K. Gladilin, ${ }^{11}$ C. Glasman, ${ }^{96}$ J. Glatzer, ${ }^{14}$ P. C. F. Glaysher, ${ }^{46}$ A. Glazov, ${ }^{46}$ M. Goblirsch-Kolb, ${ }^{26}$ J. Godlewski, ${ }^{42}$ S. Goldfarb, ${ }^{102}$ T. Golling, ${ }^{55}$ D. Golubkov, ${ }^{139}$ A. Gomes, ${ }^{135 a, 135 b, 135 d}$ R. Gonçalo, ${ }^{135 a}$ R. Goncalves Gama, ${ }^{141 \mathrm{~b}}$ G. Gonella, ${ }^{53}$ L. Gonella, ${ }^{21}$ A. Gongadze,${ }^{80}$ F. Gonnella, ${ }^{21}$ J. L. Gonski, ${ }^{60}$ S. González de la Hoz, ${ }^{171}$ S. Gonzalez-Sevilla, ${ }^{55}$ L. Goossens, ${ }^{35}$ P. A. Gorbounov, ${ }^{109}$ H. A. Gordon, ${ }^{29}$ B. Gorini, ${ }^{35}$ E. Gorini, ${ }^{68 a, 68 b}$ A. Gorišek, ${ }^{89}$ A. T. Goshaw, ${ }^{49}$ C. Gössling, ${ }^{47}$ M. I. Gostkin, ${ }^{80}$ C. A. Gottardo, ${ }^{24}$ C. R. Goudet,${ }^{128}$ D. Goujdami, ${ }^{34 c}$ A. G. Goussiou, ${ }^{145}$ N. Govender, ${ }^{32 b, b}$ C. Goy, ${ }^{5}$ E. Gozani, ${ }^{157}$ I. Grabowska-Bold, ${ }^{41 a}$ P. O. J. Gradin, ${ }^{169}$ E. C. Graham, ${ }^{88}$ J. Gramling, ${ }^{168}$ E. Gramstad, ${ }^{130}$ S. Grancagnolo, ${ }^{19}$ V. Gratchev ${ }^{133}$ P. M. Gravila, ${ }^{27 f}$ C. Gray, ${ }^{58}$ H. M. Gray, ${ }^{18}$ Z. D. Greenwood,${ }^{93, a l}$ C. Grefe, ${ }^{24}$ K. Gregersen, ${ }^{92}$ I. M. Gregor, ${ }^{46}$ P. Grenier, ${ }^{150}$ K. Grevtsov, ${ }^{46}$ J. Griffiths, ${ }^{8}$ A. A. Grillo, ${ }^{143}$ K. Grimm, ${ }^{150}$ S. Grinstein, ${ }^{14, a b}$ Ph. Gris, ${ }^{37}$ J.-F. Grivaz, ${ }^{128}$ S. Groh, ${ }^{97}$ E. Gross, ${ }^{177}$ J. Grosse-Knetter, ${ }^{54}$ G. C. Grossi, ${ }^{93}$ Z. J. Grout,${ }^{92}$ C. Grud, ${ }^{103}$ A. Grummer, ${ }^{116}$ L. Guan, ${ }^{103}$ W. Guan, ${ }^{178}$ J. Guenther, ${ }^{35}$ A. Guerguichon, ${ }^{128}$ F. Guescini, ${ }^{165 a}$ D. Guest, ${ }^{168}$ R. Gugel,${ }^{53}$ B. Gui, ${ }^{122}$ T. Guillemin, ${ }^{5}$ S. Guindon, ${ }^{35}$ U. Gul,${ }^{58}$ C. Gumpert,${ }^{35}$ J. Guo, ${ }^{61 \mathrm{c}}$ W. Guo, ${ }^{103}$ Y. Guo, ${ }^{61 a, q}$ Z. Guo, ${ }^{99}$ R. Gupta, ${ }^{43}$ S. Gurbuz, ${ }^{12 \mathrm{c}}$ G. Gustavino, ${ }^{124}$ B. J. Gutelman, ${ }^{157}$ P. Gutierrez, ${ }^{124}$ C. Gutschow, ${ }^{92}$ C. Guyot, ${ }^{142}$ M. P. Guzik, ${ }^{41 a}$ C. Gwenlan, ${ }^{131}$ C. B. Gwilliam, ${ }^{88}$ A. Haas, ${ }^{121}$ C. Haber, ${ }^{18}$ H. K. Hadavand, ${ }^{8}$ N. Haddad, ${ }^{34 \mathrm{e}}$ A. Hadef ${ }^{61 a}$ S. Hageböck, ${ }^{24}$ M. Hagihara, ${ }^{166}$ H. Hakobyan, ${ }^{181, \dagger}$ M. Haleem, ${ }^{174}$ J. Haley, ${ }^{125}$ G. Halladjian, ${ }^{104}$ G. D. Hallewell, ${ }^{99}$ K. Hamacher, ${ }^{179}$ P. Hamal, ${ }^{126}$ K. Hamano, ${ }^{173}$ A. Hamilton, ${ }^{32 \mathrm{a}}$ G. N. Hamity, ${ }^{146}$ K. Han,${ }^{61 \mathrm{a}, \mathrm{ak}}$ L. Han,${ }^{61 \mathrm{a}}$ S. Han, ${ }^{15 \mathrm{~d}}$ K. Hanagaki, ${ }^{81, \mathrm{x}}$ M. Hance, ${ }^{143}$ D. M. Handl, ${ }^{112}$ B. Haney, ${ }^{132}$ R. Hankache, ${ }^{94}$ P. Hanke, ${ }^{62 a}$ E. Hansen, ${ }^{95}$ J. B. Hansen, ${ }^{39}$ J. D. Hansen, ${ }^{39}$ M. C. Hansen, ${ }^{24}$ P. H. Hansen, ${ }^{39}$ K. Hara, ${ }^{166}$ A. S. Hard ${ }^{178}$ T. Harenberg, ${ }^{179}$ S. Harkusha, ${ }^{105}$ P. F. Harrison, ${ }^{175}$ N. M. Hartmann, ${ }^{112}$ Y. Hasegawa, ${ }^{147}$ A. Hasib,${ }^{50}$ S. Hassani, ${ }^{142}$ S. Haug ${ }^{20}$ R. Hauser, ${ }^{104}$ L. Hauswald, ${ }^{48}$ L. B. Havener, ${ }^{38}$ M. Havranek, ${ }^{137}$ C. M. Hawkes, ${ }^{21}$ R. J. Hawkings,${ }^{35}$ D. Hayden, ${ }^{104}$ C. Hayes, ${ }^{152}$ C. P. Hays,${ }^{131}$ J. M. Hays, ${ }^{90}$ H. S. Hayward, ${ }^{88}$ S. J. Haywood, ${ }^{140}$ M. P. Heath, ${ }^{50}$ V. Hedberg, ${ }^{95}$ L. Heelan, ${ }^{8}$ S. Heer, ${ }^{24}$ K. K. Heidegger, ${ }^{53}$ J. Heilman, ${ }^{33}$ S. Heim, ${ }^{46}$ T. Heim, ${ }^{18}$ B. Heinemann, ${ }^{46, u}$ J. J. Heinrich, ${ }^{112}$ L. Heinrich, ${ }^{121}$ C. Heinz,${ }^{57}$ J. Hejbal,,${ }^{136}$ L. Helary, ${ }^{35}$ A. Held, ${ }^{172}$ S. Hellesund, ${ }^{130}$ S. Hellman, ${ }^{45 a, 45 b}$ C. Helsens, ${ }^{35}$ R. C. W. Henderson, ${ }^{87}$ Y. Heng, ${ }^{178}$ S. Henkelmann, ${ }^{172}$

A. M. Henriques Correia, ${ }^{35}$ G. H. Herbert, ${ }^{19}$ H. Herde, ${ }^{26}$ V. Herget, ${ }^{174}$ Y. Hernández Jiménez,${ }^{32 c}$ H. Herr, ${ }^{97}$ G. Herten, ${ }^{53}$ R. Hertenberger, ${ }^{112}$ L. Hervas, ${ }^{35}$ T. C. Herwig, ${ }^{132}$ G. G. Hesketh, ${ }^{92}$ N. P. Hessey, ${ }^{165 a}$ J. W. Hetherly, ${ }^{43}$ S. Higashino, ${ }^{81}$ E. Higón-Rodriguez, ${ }^{171}$ K. Hildebrand, ${ }^{36}$ E. Hill, ${ }^{173}$ J. C. Hill, ${ }^{31}$ K. K. Hill, ${ }^{29}$ K. H. Hiller, ${ }^{46}$ S. J. Hillier, ${ }^{21}$ M. Hils, ${ }^{48}$ I. Hinchliffe, ${ }^{18}$ M. Hirose, ${ }^{129}$ D. Hirschbuehl, ${ }^{179}$ B. Hiti, ${ }^{89}$ O. Hladik, ${ }^{136}$ D. R. Hlaluku, ${ }^{32 c}$ X. Hoad, ${ }^{50}$ J. Hobbs, ${ }^{152}$ N. Hod, ${ }^{165 a}$ M. C. Hodgkinson, ${ }^{146}$ A. Hoecker, ${ }^{35}$ M. R. Hoeferkamp, ${ }^{116}$ F. Hoenig, ${ }^{112}$ D. Hohn,${ }^{24}$ D. Hohov, ${ }^{128}$ T. R. Holmes, ${ }^{36}$ M. Holzbock, ${ }^{112}$ M. Homann, ${ }^{47}$ S. Honda, ${ }^{166}$ T. Honda, ${ }^{81}$ T. M. Hong, ${ }^{134}$ A. Hönle, ${ }^{113}$ B. H. Hooberman,${ }^{170}$ W. H. Hopkins ${ }^{127}$ Y. Horii, ${ }^{15}$ P. Horn ${ }^{48}$ A. J. Horton, ${ }^{149}$ L. A. Horyn, ${ }^{36}$ J-Y. Hostachy, ${ }^{59}$ A. Hostiuc, ${ }^{145}$ S. Hou, ${ }^{155}$ A. Hoummada, ${ }^{34 \mathrm{a}}$ J. Howarth, ${ }^{98}$ J. Hoya,${ }^{86}$ M. Hrabovsky, ${ }^{126}$ J. Hrdinka, ${ }^{35}$ I. Hristova, ${ }^{19}$ J. Hrivnac, ${ }^{128}$ A. Hrynevich, ${ }^{106}$ T. Hryn'ova, ${ }^{5}$ P. J. Hsu, ${ }^{65}$ S.-C. Hsu, ${ }^{145}$ Q. Hu, ${ }^{29}$ S. Hu,${ }^{61 \mathrm{c}}$ Y. Huang, ${ }^{15 \mathrm{a}}$ Z. Hubacek,${ }^{137}$ F. Hubaut, ${ }^{99}$ M. Huebner, ${ }^{24}$ F. Huegging, ${ }^{24}$ T. B. Huffman, ${ }^{131}$ E. W. Hughes ${ }^{38}$ M. Huhtinen, ${ }^{35}$ R. F. H. Hunter, ${ }^{33}$ P. Huo, ${ }^{152}$ A. M. Hupe,${ }^{33}$

N. Huseynov, ${ }^{80, a i}$ J. Huston, ${ }^{104}$ J. Huth,${ }^{60}$ R. Hyneman, ${ }^{103}$ G. Iacobucci, ${ }^{55}$ G. Iakovidis, ${ }^{29}$ I. Ibragimov, ${ }^{148}$

L. Iconomidou-Fayard, ${ }^{128}$ Z. Idrissi, ${ }^{34 \mathrm{e}} \mathrm{P}$. Iengo,${ }^{35} \mathrm{R}$. Ignazzi, ${ }^{39}$ O. Igonkina, ${ }^{18, \text { ad }} \mathrm{R}$. Iguchi, ${ }^{160} \mathrm{~T}$. Iizawa, ${ }^{55}$ Y. Ikegami ${ }^{81}$ M. Ikeno, ${ }^{81} \mathrm{D}$. Iliadis, ${ }^{159} \mathrm{~N}$. Ilic, ${ }^{150} \mathrm{~F}$. Iltzsche, ${ }^{48} \mathrm{G}$. Introzzi, ${ }^{71,71 \mathrm{~b}} \mathrm{M}$. Iodice, ${ }^{75 \mathrm{a}} \mathrm{K}$. Iordanidou, ${ }^{38} \mathrm{~V}$. Ippolito, ${ }^{73 \mathrm{a}, 73 \mathrm{~b}}$ M. F. Isacson, ${ }^{169} \mathrm{~N}$. Ishijima, ${ }^{129} \mathrm{M}$. Ishino, ${ }^{160} \mathrm{M}$. Ishitsuka, ${ }^{162} \mathrm{~W}$. Islam, ${ }^{125} \mathrm{C}$. Issever, ${ }^{131} \mathrm{~S}$. Istin, ${ }^{12 \mathrm{c}, \mathrm{ap}} \mathrm{F}$. Ito, ${ }^{166}$ J. M. Iturbe Ponce, ${ }^{64 a}$ R. Iuppa ${ }^{76 a, 76 b}$ A. Ivina, ${ }^{177}$ H. Iwasaki, ${ }^{81}$ J. M. Izen, ${ }^{44}$ V. Izzo, ${ }^{70 a}$ S. Jabbar, ${ }^{3}$ P. Jacka, ${ }^{136}$ P. Jackson, ${ }^{1}$ R. M. Jacobs ${ }^{24}$ V. Jain, ${ }^{2}$ G. Jäkel, ${ }^{179}$ K. B. Jakobi,${ }^{97}$ K. Jakobs,${ }^{53}$ S. Jakobsen, ${ }^{77}$ T. Jakoubek, ${ }^{136}$ D. O. Jamin, ${ }^{125}$ D. K. Jana ${ }^{93}$ R. Jansky, ${ }^{55}$ J. Janssen,${ }^{24}$ M. Janus, ${ }^{54}$ P. A. Janus, ${ }^{41 a}$ G. Jarlskog, ${ }^{95}$ N. Javadov, ${ }^{80, a i}$ T. Javůrek, ${ }^{53}$ M. Javurkova, ${ }^{53}$ F. Jeanneau, ${ }^{142}$ L. Jeanty ${ }_{18}^{18}$ J. Jejelava, ${ }^{156 a, a j}$ A. Jelinskas, ${ }^{175}$ P. Jenni,${ }^{53, c}$ J. Jeong,${ }^{46}$ C. Jeske, ${ }^{175}$ S. Jézéquel, ${ }^{5}$ H. Ji, ${ }^{178}$ J. Jia, ${ }^{152}$ H. Jiang, ${ }^{79}$ Y. Jiang, ${ }^{61 \mathrm{a}}$ Z. Jiang, ${ }^{150}$ S. Jiggins, ${ }^{53}$ F. A. Jimenez Morales, ${ }^{37}$ J. Jimenez Pena, ${ }^{171}$ S. Jin, ${ }^{15 b}$ 
A. Jinaru, ${ }^{27 b}$ O. Jinnouchi, ${ }^{162}$ H. Jivan, ${ }^{32 c}$ P. Johansson, ${ }^{146}$ K. A. Johns, ${ }^{7}$ C. A. Johnson, ${ }^{66}$ W. J. Johnson, ${ }^{145}$ K. Jon-And, ${ }^{45 a, 45 b}$ R. W. L. Jones, ${ }^{87}$ S. D. Jones, ${ }^{153}$ S. Jones, ${ }^{7}$ T. J. Jones, ${ }^{88}$ J. Jongmanns, ${ }^{62 a}$ P. M. Jorge, ${ }^{135 a, 135 b}$ J. Jovicevic, ${ }^{165 a}$ X. Ju, ${ }^{178}$ J. J. Junggeburth, ${ }^{113}$ A. Juste Rozas, ${ }^{14, a b}$ A. Kaczmarska, ${ }^{42}$ M. Kado, ${ }^{128}$ H. Kagan, ${ }^{122}$ M. Kagan, ${ }^{150}$ T. Kaji, ${ }^{176}$ E. Kajomovitz, ${ }^{157}$ C. W. Kalderon, ${ }^{95}$ A. Kaluza, ${ }^{97}$ S. Kama, ${ }^{43}$ A. Kamenshchikov, ${ }^{139}$ L. Kanjir, ${ }^{89}$ Y. Kano, ${ }^{160}$ V. A. Kantserov, ${ }^{110}$ J. Kanzaki, ${ }^{81}$ B. Kaplan, ${ }^{121}$ L. S. Kaplan, ${ }^{178}$ D. Kar, ${ }^{32 \mathrm{c}}$ M. J. Kareem, ${ }^{165 \mathrm{~b}}$ E. Karentzos, ${ }^{10}$ S. N. Karpov, ${ }^{80}$ Z. M. Karpova ${ }^{80}$ V. Kartvelishvili, ${ }^{87}$ A. N. Karyukhin, ${ }^{139}$ K. Kasahara, ${ }^{166}$ L. Kashif, ${ }^{178}$ R. D. Kass, ${ }^{122}$ A. Kastanas, ${ }^{151}$

Y. Kataoka, ${ }^{160}$ C. Kato, ${ }^{160}$ J. Katzy, ${ }^{46}$ K. Kawade,${ }^{82}$ K. Kawagoe, ${ }^{85}$ T. Kawamoto, ${ }^{160}$ G. Kawamura, ${ }^{54}$ E. F. Kay, ${ }^{88}$ V. F. Kazanin, ${ }^{120 b, 120 a}$ R. Keeler, ${ }^{173}$ R. Kehoe, ${ }^{43}$ J. S. Keller, ${ }^{33}$ E. Kellermann, ${ }^{95}$ J. J. Kempster, ${ }^{21}$ J. Kendrick, ${ }^{21}$ O. Kepka, ${ }^{136}$ S. Kersten, ${ }^{179}$ B. P. Kerševan, ${ }^{89}$ R. A. Keyes, ${ }^{101}$ M. Khader, ${ }^{170}$ F. Khalil-zada, ${ }^{13}$ A. Khanov, ${ }^{125}$ A. G. Kharlamov, ${ }^{120 b, 120 a}$ T. Kharlamova, ${ }^{120 b, 120 a}$ A. Khodinov, ${ }^{163}$ T. J. Khoo, ${ }^{55}$ E. Khramov, ${ }^{80}$ J. Khubua, ${ }^{156 b, v}$ S. Kido, ${ }^{82}$ M. Kiehn, ${ }^{55}$ C. R. Kilby, ${ }^{91}$ S. H. Kim, ${ }^{166}$ Y. K. Kim, ${ }^{36}$ N. Kimura, ${ }^{67,67 c}$ O. M. Kind, ${ }^{19}$ B. T. King, ${ }^{88}$ D. Kirchmeier, ${ }^{48}$ J. Kirk, ${ }^{140}$ A. E. Kiryunin, ${ }^{113}$ T. Kishimoto, ${ }^{160}$ D. Kisielewska, ${ }^{41 a}$ V. Kitali, ${ }^{46}$ O. Kivernyk, ${ }^{5}$ E. Kladiva, ${ }^{28 b}$ T. Klapdor-Kleingrothaus, ${ }^{53}$ M. H. Klein, ${ }^{103}$ M. Klein ${ }^{88}$ U. Klein, ${ }^{88}$ K. Kleinknecht,${ }^{97}$ P. Klimek, ${ }^{119}$ A. Klimentov, ${ }^{29}$ R. Klingenberg, ${ }^{47, \dagger}$ T. Klingl, ${ }^{24}$

T. Klioutchnikova, ${ }^{35}$ F. F. Klitzner, ${ }^{112}$ P. Kluit, ${ }^{118}$ S. Kluth, ${ }^{113}$ E. Kneringer, ${ }^{77}$ E. B. F. G. Knoops, ${ }^{99}$ A. Knue, ${ }^{53}$ A. Kobayashi, ${ }^{160}$ D. Kobayashi, ${ }^{85}$ T. Kobayashi, ${ }^{160}$ M. Kobel,${ }^{48}$ M. Kocian, ${ }^{150}$ P. Kodys, ${ }^{138}$ T. Koffas, ${ }^{33}$ E. Koffeman, ${ }^{118}$ N. M. Köhler, ${ }^{113}$ T. Koi ${ }^{150}$ M. Kolb, ${ }^{62 b}$ I. Koletsou, ${ }^{5}$ T. Kondo,${ }^{81}$ N. Kondrashova, ${ }^{61 c}$ K. Köneke, ${ }^{53}$ A. C. König, ${ }^{117}$ T. Kono, ${ }^{81}$ R. Konoplich, ${ }^{121, \text { am }}$ V. Konstantinides, ${ }^{92}$ N. Konstantinidis, ${ }^{92}$ B. Konya, ${ }^{95}$ R. Kopeliansky, ${ }^{66}$ S. Koperny, ${ }^{41 a}$ K. Korcyl, ${ }^{42}$ K. Kordas, ${ }^{159}$ A. Korn, ${ }^{92}$ I. Korolkov, ${ }^{14}$ E. V. Korolkova, ${ }^{146}$ O. Kortner, ${ }^{113}$ S. Kortner, ${ }^{113}$ T. Kosek, ${ }^{138}$ V. V. Kostyukhin, ${ }^{24}$ A. Kotwal, ${ }^{49}$ A. Koulouris, ${ }^{10}$ A. Kourkoumeli-Charalampidi, ${ }^{71 a, 71 b}$ C. Kourkoumelis, ${ }^{9}$ E. Kourlitis, ${ }^{146}$ V. Kouskoura, ${ }^{29}$ A. B. Kowalewska, ${ }^{42}$ R. Kowalewski, ${ }^{173}$ T. Z. Kowalski, ${ }^{41 a}$ C. Kozakai, ${ }^{160}$ W. Kozanecki, ${ }^{142}$ A. S. Kozhin, ${ }^{139}$ V. A. Kramarenko, ${ }^{111}$ G. Kramberger, ${ }^{89}$ D. Krasnopevtsev, ${ }^{110}$ M. W. Krasny, ${ }^{94}$ A. Krasznahorkay, ${ }^{35}$ D. Krauss, ${ }^{113}$ J. A. Kremer, ${ }^{41 a}$ J. Kretzschmar, ${ }^{88}$ P. Krieger, ${ }^{164}$ K. Krizka, ${ }^{18}$ K. Kroeninger, ${ }^{47}$ H. Kroha, ${ }^{113}$ J. Kroll, ${ }^{136}$ J. Kroll, ${ }^{132}$ J. Krstic, ${ }^{16}$ U. Kruchonak, ${ }^{80}$ H. Krüger, ${ }^{24}$ N. Krumnack, ${ }^{79}$ M. C. Kruse, ${ }^{49}$ T. Kubota, ${ }^{102}$ S. Kuday, ${ }^{4 b}$ J. T. Kuechler, ${ }^{179}$ S. Kuehn, ${ }^{35}$ A. Kugel, ${ }^{62 a}$ F. Kuger, ${ }^{174}$ T. Kuhl, ${ }^{46}$ V. Kukhtin, ${ }^{80}$ R. Kukla, ${ }^{99}$ Y. Kulchitsky, ${ }^{105}$ S. Kuleshov, ${ }^{14 \mathrm{~b}}$ Y. P. Kulinich, ${ }^{170}$ M. Kuna, ${ }^{59}$ T. Kunigo, ${ }^{83}$ A. Kupco, ${ }^{136}$ T. Kupfer, ${ }^{47}$ O. Kuprash, ${ }^{158}$ H. Kurashige ${ }^{82}$ L. L. Kurchaninov, ${ }^{165 a}$ Y. A. Kurochkin, ${ }^{105}$ M. G. Kurth, ${ }^{15 \mathrm{~d}}$ E. S. Kuwertz, ${ }^{173}$ M. Kuze, ${ }^{162}$ J. Kvita, ${ }^{126}$ T. Kwan, ${ }^{101}$ A. La Rosa, ${ }^{113}$ J. L. La Rosa Navarro, ${ }^{141 \mathrm{~d}}$ L. La Rotonda,${ }^{40 b, 40 a}$ F. La Ruffa,${ }^{40 b, 40 a}$ C. Lacasta, ${ }^{171}$ F. Lacava, ${ }^{73 a, 73 b}$ J. Lacey, ${ }^{46}$ D. P. J. Lack, ${ }^{98}$ H. Lacker, ${ }^{19}$ D. Lacour, ${ }^{94}$ E. Ladygin,${ }^{80}$ R. Lafaye, ${ }^{5}$ B. Laforge,${ }^{94}$ T. Lagouri, ${ }^{32 \mathrm{c}}$ S. Lai,${ }^{54}$ S. Lammers,${ }^{66}$ W. Lampl, ${ }^{7}$ E. Lançon, ${ }^{29}$ U. Landgraf, ${ }^{53}$ M. P. J. Landon, ${ }^{90}$ M. C. Lanfermann, ${ }^{55}$ V. S. Lang, ${ }^{46}$ J. C. Lange, ${ }^{14}$ R. J. Langenberg, ${ }^{35}$ A. J. Lankford, ${ }^{168}$ F. Lanni, ${ }^{29}$ K. Lantzsch, ${ }^{24}$ A. Lanza, ${ }^{71 a}$ A. Lapertosa, ${ }^{56,56 a}$ S. Laplace, ${ }^{94}$ J. F. Laporte, ${ }^{142}$ T. Lari, ${ }^{69 a}$ F. Lasagni Manghi, ${ }^{23 b, 23 a}$ M. Lassnig, ${ }^{35}$ T. S. Lau, ${ }^{64 a}$ A. Laudrain, ${ }^{128}$ A. T. Law, ${ }^{143}$ P. Laycock, ${ }^{88}$ M. Lazzaroni, ${ }^{69 a, 69 b}$ B. Le, ${ }^{102}$ O. Le Dortz, ${ }^{94}$ E. Le Guirriec, ${ }^{99}$ E. P. Le Quilleuc, ${ }^{142}$ M. LeBlanc, ${ }^{7}$ T. LeCompte ${ }^{6}$ F. Ledroit-Guillon, ${ }^{59}$ C. A. Lee, ${ }^{29}$ G. R. Lee, ${ }^{144 a}$ L. Lee ${ }^{60}$ S. C. Lee, ${ }^{155}$ B. Lefebvre, ${ }^{101}$ M. Lefebvre, ${ }^{173}$ F. Legger, ${ }^{112}$ C. Leggett, ${ }^{18}$ N. Lehmann, ${ }^{179}$ G. Lehmann Miotto, ${ }^{35}$ W. A. Leight, ${ }^{46}$ A. Leisos, ${ }^{159, y}$ M. A. L. Leite, ${ }^{141 d}$ R. Leitner, ${ }^{138}$ D. Lellouch, ${ }^{177}$ B. Lemmer, ${ }^{54}$ K. J. C. Leney, ${ }^{92}$ T. Lenz,${ }^{24}$ B. Lenzi, ${ }^{35}$ R. Leone, ${ }^{7}$ S. Leone, ${ }^{72 a}$ C. Leonidopoulos,${ }^{50}$ G. Lerner, ${ }^{153}$ C. Leroy, ${ }^{107}$ R. Les, ${ }^{164}$ A. A. J. Lesage, ${ }^{142}$ C. G. Lester, ${ }^{31}$ M. Levchenko, ${ }^{133}$ J. Levêque, ${ }^{5}$ D. Levin, ${ }^{103}$ L. J. Levinson, ${ }^{177}$ D. Lewis, ${ }^{90}$ B. Li, ${ }^{103}$ C.-Q. Li, ${ }^{61 \mathrm{a}} \mathrm{H} . \mathrm{Li},{ }^{61 \mathrm{~b}} \mathrm{~L} . \mathrm{Li},{ }^{61 \mathrm{c}} \mathrm{Q} . \mathrm{Li},{ }^{15 \mathrm{~d}} \mathrm{Q} . \mathrm{Li},{ }^{61 \mathrm{a}} \mathrm{S} . \mathrm{Li},{ }^{61 \mathrm{~d}, 61 \mathrm{c}} \mathrm{X} . \mathrm{Li},{ }^{61 \mathrm{c}} \mathrm{Y} . \mathrm{Li}^{148}$ Z. Liang, ${ }^{15}$ B. Liberti, ${ }^{74 a}$ A. Liblong, ${ }^{164}$ K. Lie, ${ }^{64 \mathrm{c}}$ S. Liem, ${ }^{118}$ A. Limosani, ${ }^{154}$ C. Y. Lin, ${ }^{31}$ K. Lin, ${ }^{104}$ T. H. Lin, ${ }^{97}$ R. A. Linck, ${ }^{66}$ B. E. Lindquist, ${ }^{152}$ A. L. Lionti, ${ }^{55}$ E. Lipeles, ${ }^{132}$ A. Lipniacka, ${ }^{17}$ M. Lisovyi, ${ }^{62 b}$ T. M. Liss, ${ }^{170, a r}$ A. Lister, ${ }^{172}$ A. M. Litke, ${ }^{143}$ J. D. Little, ${ }^{8}$ B. Liu, ${ }^{79}$ B. L Liu,${ }^{6}$ H. Liu,${ }^{29}$ H. Liu, ${ }^{103}$ J. B. Liu,${ }^{61 a}$ J. K. K. Liu, ${ }^{131}$ K. Liu,${ }^{94}$ M. Liu, ${ }^{61 a}$ P. Liu,${ }^{18}$ Y. Liu, ${ }^{61 \mathrm{a}}$ Y. Liu, ${ }^{15 \mathrm{a}}$ Y. L. Liu, ${ }^{61 \mathrm{a}}$ M. Livan, ${ }^{71 \mathrm{a}, 7 \mathrm{~b}}$ A. Lleres, ${ }^{59}$ J. Llorente Merino, ${ }^{15 \mathrm{a}}$ S. L. Lloyd, ${ }^{90}$ C. Y. Lo ${ }^{64 \mathrm{~b}}$ F. Lo Sterzo, ${ }^{43}$ E. M. Lobodzinska, ${ }^{46}$ P. Loch, ${ }^{7}$ F. K. Loebinger,${ }^{98}$ A. Loesle, ${ }^{53}$ K. M. Loew, ${ }^{26}$ T. Lohse, ${ }^{19}$ K. Lohwasser, ${ }^{146}$ M. Lokajicek, ${ }^{136}$ B. A. Long, ${ }^{25}$ J. D. Long, ${ }^{170}$ R. E. Long, ${ }^{87}$ L. Longo, ${ }^{68 a, 68 b}$ K. A. Looper ${ }^{122}$ J. A. Lopez, ${ }^{144 b}$ I. Lopez Paz, ${ }^{14}$

A. Lopez Solis, ${ }^{146}$ J. Lorenz, ${ }^{112}$ N. Lorenzo Martinez, ${ }^{5}$ M. Losada, ${ }^{22}$ P. J. Lösel, ${ }^{112}$ X. Lou, ${ }^{46}$ X. Lou, ${ }^{15 a}$ A. Lounis, ${ }^{128}$ J. Love, ${ }^{6}$ P. A. Love,${ }^{87}$ J. J. Lozano Bahilo, ${ }^{171}$ H. Lu, ${ }^{64 a}$ M. Lu, ${ }^{61 a}$ N. Lu, ${ }^{103}$ Y. J. Lu, ${ }^{65}$ H. J. Lubatti, ${ }^{145}$ C. Luci, ${ }^{73 a, 73 b}$ A. Lucotte, ${ }^{59}$ C. Luedtke, ${ }^{53}$ F. Luehring, ${ }^{66}$ I. Luise, ${ }^{94}$ W. Lukas, ${ }^{77}$ L. Luminari, ${ }^{73 a}$ B. Lund-Jensen, ${ }^{151}$ M. S. Lutz, ${ }^{100}$ P. M. Luzi,${ }^{94}$ D. Lynn, ${ }^{29}$ R. Lysak,${ }^{136}$ E. Lytken, ${ }^{95}$ F. Lyu, ${ }^{15 a}$ V. Lyubushkin, ${ }^{80}$ H. Ma, ${ }^{29}$ L. L. Ma ${ }^{61 b}$ Y. Ma, ${ }^{61 b}$ G. Maccarrone, ${ }^{52}$ A. Macchiolo, ${ }^{113}$ C. M. Macdonald, ${ }^{146}$ J. Machado Miguens, ${ }^{132}$ D. Madaffari, ${ }^{171}$ R. Madar, ${ }^{37}$ W. F. Mader ${ }^{48}$ A. Madsen, ${ }^{46}$ N. Madysa, ${ }^{48}$ J. Maeda, ${ }^{82}$ K. Maekawa, ${ }^{160}$ S. Maeland, ${ }^{17}$ T. Maeno, ${ }^{29}$ A. S. Maevskiy, ${ }^{111}$ 
V. Magerl, ${ }^{53}$ C. Maidantchik, ${ }^{141 a}$ T. Maier, ${ }^{112}$ A. Maio, ${ }^{135 a, 135 b, 135 \mathrm{~d}}$ O. Majersky, ${ }^{28 \mathrm{a}}$ S. Majewski, ${ }^{127}$ Y. Makida, ${ }^{81}$ N. Makovec, ${ }^{128}$ B. Malaescu, ${ }^{94}$ Pa. Malecki, ${ }^{42}$ V. P. Maleev, ${ }^{133}$ F. Malek, ${ }^{59}$ U. Mallik, ${ }^{78}$ D. Malon, ${ }^{6}$ C. Malone, ${ }^{31}$ S. Maltezos, ${ }^{10}$ S. Malyukov, ${ }^{35}$ J. Mamuzic, ${ }^{171}$ G. Mancini,${ }^{52}$ I. Mandić, ${ }^{89}$ J. Maneira, ${ }^{135 a, 135 b}$

L. Manhaes de Andrade Filho, ${ }^{141 \mathrm{~b}}$ J. Manjarres Ramos, ${ }^{48}$ K. H. Mankinen, ${ }^{95}$ A. Mann, ${ }^{112}$ A. Manousos, ${ }^{77}$ B. Mansoulie, ${ }^{142}$ J. D. Mansour, ${ }^{15 a}$ M. Mantoani, ${ }^{54}$ S. Manzoni,${ }^{6 a, 69 b}$ G. Marceca, ${ }^{30}$ L. March,${ }^{55}$ L. Marchese,${ }^{131}$ G. Marchiori, ${ }^{94}$ M. Marcisovsky, ${ }^{136}$ C. A. Marin Tobon, ${ }^{35}$ M. Marjanovic, ${ }^{37}$ D. E. Marley, ${ }^{103}$ F. Marroquim, ${ }^{141 a}$ Z. Marshall, ${ }^{18}$ M. U. F Martensson, ${ }^{169}$ S. Marti-Garcia, ${ }^{171}$ C. B. Martin, ${ }^{122}$ T. A. Martin, ${ }^{175}$ V. J. Martin, ${ }^{50}$ B. Martin dit Latour, ${ }^{17}$ M. Martinez, ${ }^{14, a b}$ V. I. Martinez Outschoorn, ${ }^{100}$ S. Martin-Haugh, ${ }^{140}$ V. S. Martoiu, ${ }^{27 b}$ A. C. Martyniuk, ${ }^{92}$ A. Marzin,${ }^{35}$ L. Masetti, ${ }^{97}$ T. Mashimo, ${ }^{160}$ R. Mashinistov, ${ }^{108}$ J. Masik, ${ }^{98}$ A. L. Maslennikov, ${ }^{120 b, 120 a}$ L. H. Mason, ${ }^{102}$ L. Massa, ${ }^{74 a, 74 b}$ P. Mastrandrea, ${ }^{5}$ A. Mastroberardino, ${ }^{40 b, 40 a}$ T. Masubuchi, ${ }^{160}$ P. Mättig, ${ }^{179}$ J. Maurer, ${ }^{27 b}$ B. Maček, ${ }^{89}$ S. J. Maxfield, ${ }^{88}$

D. A. Maximov, ${ }^{120 b, 120 a}$ R. Mazini, ${ }^{155}$ I. Maznas, ${ }^{159}$ S. M. Mazza, ${ }^{143}$ N. C. Mc Fadden, ${ }^{116}$ G. Mc Goldrick, ${ }^{164}$

S. P. Mc Kee, ${ }^{103}$ A. McCarn, ${ }^{103}$ T. G. McCarthy, ${ }^{113}$ L. I. McClymont, ${ }^{92}$ E. F. McDonald, ${ }^{102}$ J. A. Mcfayden, ${ }^{35}$ G. Mchedlidze, ${ }^{54}$ M. A. McKay, ${ }^{43}$ K. D. McLean, ${ }^{173}$ S. J. McMahon, ${ }^{140}$ P. C. McNamara, ${ }^{102}$ C. J. McNicol, ${ }^{175}$ R. A. McPherson, ${ }^{173, a g}$ J. E. Mdhluli, ${ }^{32 \mathrm{c}}$ Z. A. Meadows, ${ }^{100}$ S. Meehan, ${ }^{145}$ T. Megy, ${ }^{53}$ S. Mehlhase, ${ }^{112}$ A. Mehta, ${ }^{88}$ T. Meideck, ${ }^{59}$ B. Meirose, ${ }^{44}$ D. Melini, ${ }^{171, f}$ B. R. Mellado Garcia, ${ }^{32 c}$ J. D. Mellenthin, ${ }^{54}$ M. Melo, ${ }^{28 a}$ F. Meloni, ${ }^{20}$ A. Melzer, ${ }^{24}$ S. B. Menary,${ }^{98}$ E. D. Mendes Gouveia, ${ }^{135 a}$ L. Meng, ${ }^{88}$ X. T. Meng, ${ }^{103}$ A. Mengarelli, ${ }^{23 b, 23 a}$ S. Menke, ${ }^{113}$

E. Meoni,${ }^{40 b, 40 a}$ S. Mergelmeyer, ${ }^{19}$ C. Merlassino,${ }^{20}$ P. Mermod ${ }^{55}$ L. Merola $,{ }^{70 a}, 70 b$ C. Meroni, ${ }^{69 a}$ F. S. Merritt, ${ }^{36}$ A. Messina, ${ }^{73 a, 73 b}$ J. Metcalfe, ${ }^{6}$ A. S. Mete, ${ }^{168}$ C. Meyer, ${ }^{132}$ J. Meyer, ${ }^{157}$ J-P. Meyer, ${ }^{142}$ H. Meyer Zu Theenhausen, ${ }^{62 a}$ F. Miano, ${ }^{153}$ R. P. Middleton, ${ }^{140}$ L. Mijović ${ }^{50}$ G. Mikenberg, ${ }^{177}$ M. Mikestikova, ${ }^{136}$ M. Mikuž,${ }^{89}$ M. Milesi,${ }^{102}$ A. Milic, ${ }^{164}$ D. A. Millar, ${ }^{90}$ D. W. Miller, ${ }^{36}$ A. Milov, ${ }^{177}$ D. A. Milstead, ${ }^{45 a, 45 b}$ A. A. Minaenko, ${ }^{139}$ M. Miñano Moya, ${ }^{171}$ I. A. Minashvili, ${ }^{156 \mathrm{~b}}$ A. I. Mincer, ${ }^{121}$ B. Mindur, ${ }^{41 \mathrm{a}}$ M. Mineev, ${ }^{80}$ Y. Minegishi, ${ }^{160}$ Y. Ming, ${ }^{178}$ L. M. Mir, ${ }^{14}$ A. Mirto, ${ }^{68 a, 68 b}$ K. P. Mistry, ${ }^{132}$ T. Mitani, ${ }^{176}$ J. Mitrevski, ${ }^{112}$ V. A. Mitsou, ${ }^{171}$ A. Miucci, ${ }^{20}$ P. S. Miyagawa, ${ }^{146}$ A. Mizukami, ${ }^{81}$ J. U. Mjörnmark, ${ }^{95}$ T. Mkrtchyan, ${ }^{181}$ M. Mlynarikova, ${ }^{138}$ T. Moa,${ }^{45 a, 45 b}$ K. Mochizuki, ${ }^{107}$ P. Mogg,${ }^{53}$ S. Mohapatra, ${ }^{38}$ S. Molander, ${ }^{45 a, 45 b}$ R. Moles-Valls, ${ }^{24}$ M. C. Mondragon, ${ }^{104}$ K. Mönig, ${ }^{46}$ J. Monk,${ }^{39}$ E. Monnier, ${ }^{99}$ A. Montalbano, ${ }^{149}$ J. Montejo Berlingen, ${ }^{35}$ F. Monticelli, ${ }^{86}$ S. Monzani, ${ }^{69 a}$ R. W. Moore, ${ }^{3}$ N. Morange, ${ }^{128}$ D. Moreno, ${ }^{22}$ M. Moreno Llácer, ${ }^{35}$ P. Morettini, ${ }^{56 \mathrm{~b}}$ M. Morgenstern, ${ }^{118}$ S. Morgenstern, ${ }^{35}$ D. Mori, ${ }^{149}$ T. Mori, ${ }^{160}$ M. Morii, ${ }^{60}$ M. Morinaga, ${ }^{176}$ V. Morisbak, ${ }^{130}$ A. K. Morley, ${ }^{35}$ G. Mornacchi, ${ }^{35}$ A. P. Morris,${ }^{92}$ J. D. Morris,${ }^{90}$ L. Morvaj, ${ }^{152}$ P. Moschovakos, ${ }^{10}$ M. Mosidze, ${ }^{156 b}$ H. J. Moss, ${ }^{146}$ J. Moss,${ }^{150,1}$ K. Motohashi, ${ }^{162}$ R. Mount,${ }^{150}$ E. Mountricha, ${ }^{35}$ E. J. W. Moyse, ${ }^{100}$ S. Muanza, ${ }^{99}$ F. Mueller, ${ }^{113}$ J. Mueller, ${ }^{134}$ R. S. P. Mueller, ${ }^{12}$ D. Muenstermann, ${ }^{87}$ P. Mullen, ${ }^{58}$ G. A. Mullier, ${ }^{20}$ F. J. Munoz Sanchez,${ }^{98}$ P. Murin, ${ }^{28 b}$ W. J. Murray, ${ }^{175,140}$ A. Murrone, ${ }^{69 a, 69 b}$ M. Muškinja, ${ }^{89}$ C. Mwewa, ${ }^{32 a}$ A. G. Myagkov, ${ }^{139, \text { an }}$ J. Myers, ${ }^{127}$ M. Myska, ${ }^{137}$ B. P. Nachman, ${ }^{18}$ O. Nackenhorst,${ }^{47}$ K. Nagai, ${ }^{131}$ K. Nagano, ${ }^{81}$ Y. Nagasaka ${ }^{63}$ K. Nagata, ${ }^{166}$ M. Nagel, ${ }^{53}$ E. Nagy, ${ }^{99}$ A. M. Nairz, ${ }^{35}$ Y. Nakahama, ${ }^{115}$ K. Nakamura, ${ }^{81}$ T. Nakamura, ${ }^{160}$ I. Nakano, ${ }^{123}$ H. Nanjo, ${ }^{129}$ F. Napolitano, ${ }^{62 a}$ R. F. Naranjo Garcia, ${ }^{46}$ R. Narayan, ${ }^{11}$ D. I. Narrias Villar, ${ }^{62 a}$ I. Naryshkin, ${ }^{133}$ T. Naumann, ${ }^{46}$ G. Navarro, ${ }^{22}$ R. Nayyar, H. A. Neal,${ }^{103}$ P. Yu. Nechaeva, ${ }^{108}$ T. J. Neep,${ }^{142}$ A. Negri, ${ }^{71 a, 71 b}$ M. Negrini,${ }^{23 b}$ S. Nektarijevic,,${ }^{117}$ C. Nellist, ${ }^{54}$ M. E. Nelson, ${ }^{131}$ S. Nemecek, ${ }^{136}$ P. Nemethy, ${ }^{121}$ M. Nessi, ${ }^{35, g}$ M. S. Neubauer, ${ }^{170}$ M. Neumann, ${ }^{179}$ P. R. Newman, ${ }^{21}$ T. Y. Ng ${ }^{64 \mathrm{c}}$ Y. S. Ng, ${ }^{19}$ H. D. N. Nguyen, ${ }^{99}$ T. Nguyen Manh, ${ }^{107}$ E. Nibigira, ${ }^{37}$ R. B. Nickerson, ${ }^{131}$ R. Nicolaidou, ${ }^{142}$ J. Nielsen, ${ }^{143}$ N. Nikiforou, ${ }^{11}$ V. Nikolaenko, ${ }^{139, \text { an }}$ I. Nikolic-Audit, ${ }^{94}$ K. Nikolopoulos, ${ }^{21}$ P. Nilsson, ${ }^{29}$ Y. Ninomiya ${ }^{81}$ A. Nisati, ${ }^{73 a}$ N. Nishu, ${ }^{61 \mathrm{c}}$ R. Nisius,${ }^{113}$ I. Nitsche, ${ }^{47}$ T. Nitta, ${ }^{176}$ T. Nobe,${ }^{160}$ Y. Noguchi,${ }^{83}$ M. Nomachi, ${ }^{129}$ I. Nomidis ${ }^{94}$ M. A. Nomura, ${ }^{29}$ T. Nooney, ${ }^{90}$ M. Nordberg, ${ }^{35}$ N. Norjoharuddeen, ${ }^{131}$ T. Novak ${ }^{89}$ O. Novgorodova ${ }^{48}$ R. Novotny, ${ }^{137}$ M. Nozaki, ${ }^{81}$ L. Nozka, ${ }^{126}$ K. Ntekas, ${ }^{168}$ E. Nurse, ${ }^{92}$ F. Nuti, ${ }^{102}$ F. G. Oakham, ${ }^{33, \text { au }}$ H. Oberlack, ${ }^{113}$ T. Obermann, ${ }^{24}$ J. Ocariz, ${ }^{94}$ A. Ochi, ${ }^{82}$ I. Ochoa, ${ }^{38}$ J. P. Ochoa-Ricoux, ${ }^{144 a}$ K. O'Connor, ${ }^{26}$ S. Oda, ${ }^{85}$ S. Odaka, ${ }^{81}$ A. Oh, ${ }^{98}$ S. H. Oh, ${ }^{49}$ C. C. Ohm, ${ }^{151}$ H. Oide ${ }^{56 b, 56 a}$ H. Okawa, ${ }^{166}$ Y. Okazaki, ${ }^{83}$ Y. Okumura, ${ }^{160}$ T. Okuyama, ${ }^{81}$ A. Olariu, ${ }^{27 b}$

L. F. Oleiro Seabra, ${ }^{135 a}$ S. A. Olivares Pino, ${ }^{144 a}$ D. Oliveira Damazio, ${ }^{29}$ J. L. Oliver, ${ }^{1}$ M. J. R. Olsson, ${ }^{36}$ A. Olszewski, ${ }^{42}$ J. Olszowska, ${ }^{42}$ D. C. O’Neil, ${ }^{149}$ A. Onofre, ${ }^{135 a, 135 e}$ K. Onogi, ${ }^{115}$ P. U. E. Onyisi, ${ }^{11}$ H. Oppen, ${ }^{130}$ M. J. Oreglia, ${ }^{36}$ Y. Oren, ${ }^{158}$ D. Orestano, ${ }^{75 a, 75 b}$ E. C. Orgill, ${ }^{98}$ N. Orlando, ${ }^{64 b}$ A. A. O’Rourke, ${ }^{46}$ R. S. Orr, ${ }^{164}$ B. Osculati,${ }^{56 b, 56 a, \dagger}$ V. O’Shea, ${ }^{58}$ R. Ospanov, ${ }^{61 a}$ G. Otero y Garzon, ${ }^{30}$ H. Otono, ${ }^{85}$ M. Ouchrif, ${ }^{34 d}$ F. Ould-Saada, ${ }^{130}$ A. Ouraou, ${ }^{142}$ Q. Ouyang, ${ }^{15 a}$ M. Owen, ${ }^{58}$ R. E. Owen, ${ }^{21}$ V.E. Ozcan, ${ }^{12 c}$ N. Ozturk, ${ }^{8}$ J. Pacalt, ${ }^{126}$ H. A. Pacey, ${ }^{31}$ K. Pachal, ${ }^{149}$ A. Pacheco Pages, ${ }^{14}$

L. Pacheco Rodriguez, ${ }^{142}$ C. Padilla Aranda ${ }^{14}$ S. Pagan Griso, ${ }^{18}$ M. Paganini, ${ }^{180}$ G. Palacino, ${ }^{66}$ S. Palazzo, ${ }^{40 b, 40 a}$ S. Palestini, ${ }^{35}$ M. Palka, ${ }^{41 b}$ D. Pallin, ${ }^{37}$ I. Panagoulias, ${ }^{10}$ C. E. Pandini, ${ }^{55}$ J. G. Panduro Vazquez, ${ }^{91}$ P. Pani, ${ }^{35}$ 
G. Panizzo, ${ }^{67,67 \mathrm{c}}$ L. Paolozzi, ${ }^{55}$ Th. D. Papadopoulou, ${ }^{10}$ K. Papageorgiou, ${ }^{9, \mathrm{~h}}$ A. Paramonov, ${ }^{6}$ D. Paredes Hernandez, ${ }^{64 \mathrm{~b}}$ S. R. Paredes Saenz, ${ }^{131}$ B. Parida, ${ }^{61 \mathrm{c}}$ A. J. Parker ${ }^{87}$ K. A. Parker, ${ }^{46}$ M. A. Parker, ${ }^{31}$ F. Parodi, ${ }^{56 b, 56 a}$ J. A. Parsons,${ }^{38}$ U. Parzefall, ${ }^{53}$ V. R. Pascuzzi, ${ }^{164}$ J. M. P Pasner, ${ }^{143}$ E. Pasqualucci, ${ }^{73 a}$ S. Passaggio, ${ }^{56 b}$ Fr. Pastore, ${ }^{91}$ P. Pasuwan, ${ }^{45 a, 45 b}$ S. Pataraia, ${ }^{97}$ J. R. Pater, ${ }^{98}$ A. Pathak, ${ }^{178, \mathrm{i}}$ T. Pauly, ${ }^{35}$ B. Pearson, ${ }^{113}$ M. Pedersen, ${ }^{130}$ L. Pedraza Diaz,${ }^{117}$ S. Pedraza Lopez, ${ }^{171}$ R. Pedro, ${ }^{135 a, 135 b}$ S. V. Peleganchuk, ${ }^{120 b, 120 a}$ O. Penc, ${ }^{136}$ C. Peng, ${ }^{15 d}$ H. Peng, ${ }^{61 \mathrm{a}}$ B. S. Peralva, ${ }^{14 \mathrm{~b}}$ M. M. Perego, ${ }^{142}$

A. P. Pereira Peixoto, ${ }^{135 \mathrm{a}}$ D. V. Perepelitsa, ${ }^{29}$ F. Peri, ${ }^{19}$ L. Perini, ${ }^{69 a, 69 b}$ H. Pernegger, ${ }^{35}$ S. Perrella, ${ }^{70 a, 70 b}$

V. D. Peshekhonov,${ }^{80, \dagger}$ K. Peters, ${ }^{46}$ R. F. Y. Peters, ${ }^{98}$ B. A. Petersen, ${ }^{35}$ T. C. Petersen, ${ }^{39}$ E. Petit,${ }^{59}$ A. Petridis,,${ }^{1}$ C. Petridou, ${ }^{159}$ P. Petroff, ${ }^{128}$ E. Petrolo, ${ }^{73 a}$ M. Petrov, ${ }^{131}$ F. Petrucci $,{ }^{75 a}, 75 b$ M. Pettee,${ }^{180}$ N. E. Pettersson, ${ }^{100}$ A. Peyaud, ${ }^{142}$ R. Pezoa, ${ }^{144 b}$ T. Pham, ${ }^{102}$ F. H. Phillips, ${ }^{104}$ P. W. Phillips,${ }^{140}$ G. Piacquadio, ${ }^{152}$ E. Pianori, ${ }^{18}$ A. Picazio, ${ }^{100}$ M. A. Pickering, ${ }^{131}$ R. Piegaia, ${ }^{30}$ J. E. Pilcher, ${ }^{36}$ A. D. Pilkington, ${ }^{98}$ M. Pinamonti, ${ }^{74 a, 74 b}$ J. L. Pinfold, ${ }^{3}$ M. Pitt, ${ }^{177}$ M.-A. Pleier, ${ }^{29}$ V. Pleskot, ${ }^{138}$ E. Plotnikova, ${ }^{80}$ D. Pluth, ${ }^{79}$ P. Podberezko, ${ }^{120 b, 120 a}$ R. Poettgen, ${ }^{95}$ R. Poggi,${ }^{55}$ L. Poggioli, ${ }^{128}$ I. Pogrebnyak, ${ }^{104}$ D. Pohl, ${ }^{24}$ I. Pokharel,${ }^{54}$ G. Polesello, ${ }^{71 a}$ A. Poley, ${ }^{46}$ A. Policicchio, ${ }^{40 b, 40 a}$ R. Polifka, ${ }^{35}$ A. Polini, ${ }^{23 b}$ C. S. Pollard,${ }^{46}$ V. Polychronakos,${ }^{29}$ D. Ponomarenko, ${ }^{110}$ L. Pontecorvo, ${ }^{73 a}$ G. A. Popeneciu, ${ }^{27 d}$ D. M. Portillo Quintero, ${ }^{94}$ S. Pospisil, ${ }^{137}$ K. Potamianos, ${ }^{46}$ I. N. Potrap, ${ }^{80}$ C. J. Potter,${ }^{31}$ H. Potti,${ }^{11}$ T. Poulsen,${ }^{95}$ J. Poveda, ${ }^{35}$ T. D. Powell, ${ }^{146}$ M. E. Pozo Astigarraga, ${ }^{35}$ P. Pralavorio, ${ }^{99}$ S. Prell, ${ }^{79}$ D. Price, ${ }^{98}$ M. Primavera, ${ }^{68 \mathrm{a}}$ S. Prince, ${ }^{101}$ N. Proklova, ${ }^{110}$ K. Prokofiev, ${ }^{64 \mathrm{c}}$ F. Prokoshin, ${ }^{144 \mathrm{~b}}$ S. Protopopescu, ${ }^{29}$ J. Proudfoot, ${ }^{6}$ M. Przybycien, ${ }^{41 a}$ A. Puri, ${ }^{170}$ P. Puzo, ${ }^{128}$ J. Qian, ${ }^{103}$ Y. Qin, ${ }^{98}$ A. Quadt, ${ }^{54}$ M. Queitsch-Maitland, ${ }^{46}$

A. Qureshi, ${ }^{1}$ P. Rados, ${ }^{102}$ F. Ragusa,${ }^{69 a, 69 b}$ G. Rahal,${ }^{51}$ J. A. Raine, ${ }^{98}$ S. Rajagopalan, ${ }^{29}$ T. Rashid, ${ }^{128}$ S. Raspopov, ${ }^{5}$ M. G. Ratti, ${ }^{6 a, 69 b}$ D. M. Rauch, ${ }^{46}$ F. Rauscher, ${ }^{112}$ S. Rave,${ }^{97}$ B. Ravina, ${ }^{146}$ I. Ravinovich, ${ }^{177}$ J. H. Rawling, ${ }^{98}$ M. Raymond,${ }^{35}$ A. L. Read, ${ }^{130}$ N. P. Readioff, ${ }^{59}$ M. Reale,${ }^{68 a, 68 b}$ D. M. Rebuzzi, ${ }^{71,71 b}$ A. Redelbach, ${ }^{174}$ G. Redlinger, ${ }^{29}$ R. Reece, ${ }^{143}$ R. G. Reed ${ }^{32 \mathrm{c}}$ K. Reeves, ${ }^{44}$ L. Rehnisch, ${ }^{19}$ J. Reichert, ${ }^{132}$ A. Reiss, ${ }^{97}$ C. Rembser, ${ }^{35}$ H. Ren, ${ }^{15 \mathrm{~d}}$ M. Rescigno, ${ }^{73 \mathrm{a}}$ S. Resconi, ${ }^{69 a}$ E. D. Resseguie, ${ }^{132}$ S. Rettie, ${ }^{172}$ E. Reynolds, ${ }^{21}$ O. L. Rezanova, ${ }^{120 b, 120 a}$ P. Reznicek, ${ }^{138}$ R. Richter, ${ }^{113}$ S. Richter, ${ }^{92}$ E. Richter-Was, ${ }^{41 b}$ O. Ricken, ${ }^{24}$ M. Ridel,${ }^{94}$ P. Rieck, ${ }^{113}$ C. J. Riegel, ${ }^{179}$ O. Rifki ${ }^{46}$ M. Rijssenbeek, ${ }^{152}$ A. Rimoldi, ${ }^{71 a, 71 b}$ M. Rimoldi, ${ }^{20}$ L. Rinaldi, ${ }^{23 b}$ G. Ripellino, ${ }^{151}$ B. Ristic,${ }^{87}$ E. Ritsch, ${ }^{35}$ I. Riu, ${ }^{14}$ J. C. Rivera Vergara, ${ }^{144 a}$ F. Rizatdinova, ${ }^{125}$ E. Rizvi, ${ }^{90}$ C. Rizzi,${ }^{14}$ R. T. Roberts, ${ }^{98}$ S. H. Robertson, ${ }^{101, a g}$ A. Robichaud-Veronneau, ${ }^{101}$ D. Robinson, ${ }^{31}$ J. E. M. Robinson, ${ }^{46}$ A. Robson, ${ }^{58}$ E. Rocco, ${ }^{97}$ C. Roda,${ }^{72 a, 72 b}$ Y. Rodina,${ }^{99, a c}$ S. Rodriguez Bosca, ${ }^{171}$ A. Rodriguez Perez, ${ }^{14}$

D. Rodriguez Rodriguez, ${ }^{171}$ A. M. Rodríguez Vera, ${ }^{165 b}$ S. Roe,${ }^{35}$ C. S. Rogan, ${ }^{60}$ O. Røhne, ${ }^{130}$ R. Röhrig, ${ }^{113}$

C. P. A. Roland, ${ }^{66}$ J. Roloff, ${ }^{60}$ A. Romaniouk, ${ }^{110}$ M. Romano, ${ }^{23 b, 23 a}$ N. Rompotis, ${ }^{88}$ M. Ronzani, ${ }^{121}$ L. Roos,${ }^{94}$ S. Rosati, ${ }^{73 a}$ K. Rosbach ${ }^{53}$ P. Rose, ${ }^{143}$ N.-A. Rosien, ${ }^{54}$ E. Rossi, ${ }^{70 a, 70 b}$ L. P. Rossi, ${ }^{56 b}$ L. Rossini, ${ }^{69 a, 69 b}$ J. H. N. Rosten, ${ }^{31}$ R. Rosten, ${ }^{14}$ M. Rotaru, ${ }^{27 b}$ J. Rothberg, ${ }^{145}$ D. Rousseau, ${ }^{128}$ D. Roy, ${ }^{32 \mathrm{c}}$ A. Rozanov, ${ }^{99}$ Y. Rozen,${ }^{157}$ X. Ruan,${ }^{32 \mathrm{c}}$ F. Rubbo, ${ }^{150}$ F. Rühr, ${ }^{53}$

A. Ruiz-Martinez, ${ }^{33}$ Z. Rurikova, ${ }^{53}$ N. A. Rusakovich ${ }^{80}$ H. L. Russell, ${ }^{101}$ J. P. Rutherfoord, ${ }^{7}$ N. Ruthmann, ${ }^{35}$

E. M. Rüttinger, ${ }^{46, j}$ Y. F. Ryabov, ${ }^{133}$ M. Rybar, ${ }^{170}$ G. Rybkin, ${ }^{128}$ S. Ryu, ${ }^{6}$ A. Ryzhov, ${ }^{139}$ G. F. Rzehorz, ${ }^{54}$ P. Sabatini,${ }^{54}$ G. Sabato, ${ }^{11}$ S. Sacerdoti, ${ }^{128}$ H. F-W. Sadrozinski, ${ }^{143}$ R. Sadykov, ${ }^{80}$ F. Safai Tehrani, ${ }^{73 a}$ P. Saha, ${ }^{119}$ M. Sahinsoy, ${ }^{6 a}$ A. Sahu, ${ }^{179}$ M. Saimpert, ${ }^{46}$ M. Saito,${ }^{160}$ T. Saito, ${ }^{160}$ H. Sakamoto, ${ }^{160}$ A. Sakharov, ${ }^{121, a m}$ D. Salamani, ${ }^{55}$ G. Salamanna, ${ }^{75 a, 75 b}$ J. E. Salazar Loyola, ${ }^{144 b}$ D. Salek,${ }^{118}$ P. H. Sales De Bruin, ${ }^{169}$ D. Salihagic, ${ }^{113}$ A. Salnikov, ${ }^{150}$ J. Salt, ${ }^{171}$ D. Salvatore, ${ }^{40 b, 40 a}$ F. Salvatore, ${ }^{153}$ A. Salvucci ${ }^{64 \mathrm{a}, 64 \mathrm{~b}, 64 \mathrm{c}}$ A. Salzburger, ${ }^{35}$ D. Sammel, ${ }^{53}$ D. Sampsonidis, ${ }^{159}$ D. Sampsonidou, ${ }^{159}$ J. Sánchez, ${ }^{171}$

A. Sanchez Pineda, ${ }^{67,67 \mathrm{c}}$ H. Sandaker, ${ }^{130}$ C. O. Sander, ${ }^{46}$ M. Sandhoff, ${ }^{179}$ C. Sandoval, ${ }^{22}$ D. P. C. Sankey, ${ }^{140}$

M. Sannino, ${ }^{56 b, 56 \mathrm{a}}$ Y. Sano, ${ }^{115}$ A. Sansoni ${ }^{52}$ C. Santoni, ${ }^{37}$ H. Santos, ${ }^{135 a}$ I. Santoyo Castillo, ${ }^{153}$ A. Sapronov, ${ }^{80}$ J. G. Saraiva, ${ }^{135 a, 135 d}$ O. Sasaki, ${ }^{81}$ K. Sato, ${ }^{166}$ E. Sauvan, ${ }^{5}$ P. Savard, ${ }^{164, a u}$ N. Savic, ${ }^{113}$ R. Sawada, ${ }^{160}$ C. Sawyer, ${ }^{140}$ L. Sawyer, ${ }^{93, a l}$ C. Sbarra, ${ }^{23 b}$ A. Sbrizzi, ${ }^{23 b, 23 a}$ T. Scanlon, ${ }^{92}$ J. Schaarschmidt, ${ }^{145}$ P. Schacht, ${ }^{113}$ B. M. Schachtner, ${ }^{112}$ D. Schaefer, ${ }^{36}$ L. Schaefer, ${ }^{132}$ J. Schaeffer, ${ }^{97}$ S. Schaepe,${ }^{35}$ U. Schäfer,${ }^{97}$ A. C. Schaffer, ${ }^{128}$ D. Schaile, ${ }^{112}$ R. D. Schamberger, ${ }^{152}$ N. Scharmberg, ${ }^{98}$ V. A. Schegelsky, ${ }^{133}$ D. Scheirich, ${ }^{138}$ F. Schenck, ${ }^{19}$ M. Schernau, ${ }^{168}$ C. Schiavi, ${ }^{56 b, 56 a}$ S. Schier, ${ }^{143}$ L. K. Schildgen,${ }^{24}$ Z. M. Schillaci, ${ }^{26}$ E. J. Schioppa, ${ }^{35}$ M. Schioppa, ${ }^{40 b, 40 a}$ K. E. Schleicher, ${ }^{53}$ S. Schlenker, ${ }^{35}$ K. R. Schmidt-Sommerfeld, ${ }^{113}$ K. Schmieden, ${ }^{35}$ C. Schmitt, ${ }^{97}$ S. Schmitt, ${ }^{46}$ S. Schmitz, ${ }^{97}$ U. Schnoor, ${ }^{53}$ L. Schoeffel, ${ }^{142}$ A. Schoening, ${ }^{62 b}$ E. Schopf,${ }^{24}$ M. Schott, ${ }^{97}$ J. F. P. Schouwenberg, ${ }^{117}$ J. Schovancova, ${ }^{35}$ S. Schramm, ${ }^{55}$ A. Schulte, ${ }^{97}$ H.-C. Schultz-Coulon, ${ }^{62 a}$ M. Schumacher,${ }^{53}$ B. A. Schumm, ${ }^{143}$ Ph. Schune, ${ }^{142}$ A. Schwartzman, ${ }^{150}$

T. A. Schwarz, ${ }^{103}$ H. Schweiger, ${ }^{98}$ Ph. Schwemling, ${ }^{142}$ R. Schwienhorst, ${ }^{104}$ A. Sciandra, ${ }^{24}$ G. Sciolla, ${ }^{26}$

M. Scornajenghi, ${ }^{40 b, 40 a}$ F. Scuri, ${ }^{72 a}$ F. Scutti, ${ }^{102}$ L. M. Scyboz,${ }^{113}$ J. Searcy, ${ }^{103}$ C. D. Sebastiani, ${ }^{73 a, 73 b}$ P. Seema, ${ }^{24}$

S. C. Seidel, ${ }^{116}$ A. Seiden, ${ }^{143}$ T. Seiss,${ }^{36}$ J. M. Seixas, ${ }^{141 a}$ G. Sekhniaidze, ${ }^{70 a}$ K. Sekhon, ${ }^{103}$ S. J. Sekula, ${ }^{43}$ N. Semprini-Cesari, ${ }^{23 b, 23 a}$ S. Sen, ${ }^{49}$ S. Senkin, ${ }^{37}$ C. Serfon, ${ }^{130}$ L. Serin, ${ }^{128}$ L. Serkin, ${ }^{67 a, 67 b}$ M. Sessa, ${ }^{75 a, 75 b}$ H. Severini, ${ }^{124}$ 
T. Šfiligoj, ${ }^{89}$ F. Sforza, ${ }^{167}$ A. Sfyrla,${ }^{55}$ E. Shabalina, ${ }^{54}$ J. D. Shahinian, ${ }^{143}$ N. W. Shaikh, ${ }^{45 a, 45 b}$ L. Y. Shan, ${ }^{15 a}$ R. Shang, ${ }^{170}$ J. T. Shank, ${ }^{25}$ M. Shapiro, ${ }^{18}$ A. S. Sharma, ${ }^{1}$ A. Sharma,${ }^{131}$ P. B. Shatalov, ${ }^{109}$ K. Shaw,${ }^{153}$ S. M. Shaw,${ }^{98}$ A. Shcherbakova, ${ }^{133}$ Y. Shen, ${ }^{124}$ N. Sherafati, ${ }^{33}$ A. D. Sherman, ${ }^{25}$ P. Sherwood, ${ }^{92}$ L. Shi, ${ }^{155, \text { aq }}$ S. Shimizu, ${ }^{82}$ C. O. Shimmin,${ }^{180}$ M. Shimojima, ${ }^{114}$ I. P. J. Shipsey, ${ }^{131}$ S. Shirabe, ${ }^{85}$ M. Shiyakova, ${ }^{80, a e}$ J. Shlomi, ${ }^{177}$ A. Shmeleva, ${ }^{108}$ D. Shoaleh Saadi, ${ }^{107}$ M. J. Shochet, ${ }^{36}$ S. Shojaii, ${ }^{102}$ D. R. Shope, ${ }^{124}$ S. Shrestha, ${ }^{122}$ E. Shulga, ${ }^{110}$ P. Sicho, ${ }^{136}$ A. M. Sickles, ${ }^{170}$ P. E. Sidebo, ${ }^{151}$

E. Sideras Haddad, ${ }^{32 \mathrm{c}}$ O. Sidiropoulou, ${ }^{174}$ A. Sidoti, ${ }^{23 b, 23 \mathrm{a}}$ F. Siegert, ${ }^{48}$ Dj. Sijacki, ${ }^{16}$ J. Silva, ${ }^{135 a, 135 d}$ M. Silva Jr. ${ }^{178}$ M. V. Silva Oliveira, ${ }^{141 b}$ S. B. Silverstein, ${ }^{45 a}$ L. Simic, ${ }^{80}$ S. Simion, ${ }^{128}$ E. Simioni,${ }^{97}$ M. Simon,${ }^{97}$ P. Sinervo, ${ }^{164}$ N. B. Sinev, ${ }^{127}$ M. Sioli, ${ }^{23 b, 23 a}$ G. Siragusa, ${ }^{174}$ I. Siral, ${ }^{103}$ S. Yu. Sivoklokov, ${ }^{111}$ J. Sjölin, ${ }^{45 a, 45 b}$ M. B. Skinner,${ }^{87}$ P. Skubic, ${ }^{124}$ M. Slater, ${ }^{21}$ T. Slavicek, ${ }^{137}$ M. Slawinska, ${ }^{42}$ K. Sliwa, ${ }^{167}$ R. Slovak, ${ }^{138}$ V. Smakhtin, ${ }^{177}$ B. H. Smart, ${ }^{5}$ J. Smiesko, ${ }^{28 a}$ N. Smirnov, ${ }^{110}$ S. Yu. Smirnov, ${ }^{110}$ Y. Smirnov, ${ }^{110}$ L. N. Smirnova, ${ }^{11, t}$ O. Smirnova, ${ }^{95}$ J. W. Smith, ${ }^{54}$ M. N. K. Smith, ${ }^{38}$ R. W. Smith ${ }^{38}$ M. Smizanska, ${ }^{87}$ K. Smolek, ${ }^{137}$ A. A. Snesarev,${ }^{108}$ I. M. Snyder, ${ }^{127}$ S. Snyder, ${ }^{29}$ R. Sobie, ${ }^{173, \text { ag }}$ A. M. Soffa, ${ }^{168}$ A. Soffer ${ }^{158}$ A. Søgaard, ${ }^{50}$ D. A. Soh, ${ }^{155}$ G. Sokhrannyi, ${ }^{89}$ C. A. Solans Sanchez,${ }^{35}$ M. Solar, ${ }^{137}$ E. Yu. Soldatov, ${ }^{110}$ U. Soldevila, ${ }^{171}$ A. A. Solodkov, ${ }^{139}$ A. Soloshenko, ${ }^{80}$ O. V. Solovyanov, ${ }^{139}$ V. Solovyev, ${ }^{133}$ P. Sommer, ${ }^{146}$ H. Son, ${ }^{167}$ W. Song, ${ }^{140}$ A. Sopczak, ${ }^{137}$ F. Sopkova, ${ }^{28 b}$ D. Sosa, ${ }^{62 b}$ C. L. Sotiropoulou, ${ }^{72 a, 72 b}$ S. Sottocornola, ${ }^{71 a, 71 b}$ R. Soualah, ${ }^{67 a, 67 c}$ A. M. Soukharev, ${ }^{120 b, 120 \mathrm{a}}$ D. South, ${ }^{46}$ B. C. Sowden, ${ }^{91}$ S. Spagnolo, ${ }^{68 a, 68 b}$ M. Spalla, ${ }^{113}$ M. Spangenberg, ${ }^{175}$ F. Spanò,${ }^{91}$ D. Sperlich ${ }^{19}$ F. Spettel, ${ }^{113}$ T. M. Spieker,${ }^{62 a}$ R. Spighi, ${ }^{23 b}$ G. Spigo, ${ }^{35}$ L. A. Spiller, ${ }^{102}$ D. P. Spiteri ${ }^{58}$ M. Spousta, ${ }^{138}$ A. Stabile,${ }^{69 a, 69 b}$ R. Stamen, ${ }^{62 a}$ S. Stamm, ${ }^{19}$ E. Stanecka, ${ }^{42}$ R. W. Stanek, ${ }^{6}$ C. Stanescu,${ }^{75 a}$ B. Stanislaus,${ }^{131}$ M. M. Stanitzki, ${ }^{46}$ B. S. Stapf, ${ }^{118}$ S. Stapnes, ${ }^{130}$ E. A. Starchenko, ${ }^{139}$ G. H. Stark, ${ }^{36}$ J. Stark, ${ }^{59}$ S. H Stark, ${ }^{39}$ P. Staroba, ${ }^{136}$ P. Starovoitov, ${ }^{62 a}$ S. Stärz, ${ }^{35}$ R. Staszewski, ${ }^{42}$ M. Stegler, ${ }^{46}$ P. Steinberg, ${ }^{29}$ B. Stelzer,${ }^{149}$ H. J. Stelzer, ${ }^{35}$ O. Stelzer-Chilton, ${ }^{165 a}$ H. Stenzel, ${ }^{57}$

T. J. Stevenson, ${ }^{90}$ G. A. Stewart, ${ }^{58}$ M. C. Stockton, ${ }^{127}$ G. Stoicea, ${ }^{27 b}$ P. Stolte, ${ }^{54}$ S. Stonjek, ${ }^{113}$ A. Straessner, ${ }^{48}$

J. Strandberg, ${ }^{151}$ S. Strandberg, ${ }^{45 a, 45 b}$ M. Strauss, ${ }^{124}$ P. Strizenec, ${ }^{28 b}$ R. Ströhmer, ${ }^{174}$ D. M. Strom, ${ }^{127}$ R. Stroynowski, ${ }^{43}$ A. Strubig, ${ }^{50}$ S. A. Stucci, ${ }^{29}$ B. Stugu, ${ }^{17}$ J. Stupak, ${ }^{124}$ N. A. Styles, ${ }^{46}$ D. Su, ${ }^{150}$ J. Su, ${ }^{134}$ S. Suchek, ${ }^{62 a}$ Y. Sugaya, ${ }^{129}$ M. Suk, ${ }^{137}$ V. V. Sulin, ${ }^{108}$ D. M. S. Sultan, ${ }^{55}$ S. Sultansoy, ${ }^{4 c}$ T. Sumida, ${ }^{83}$ S. Sun, ${ }^{103}$ X. Sun, ${ }^{3}$ K. Suruliz, ${ }^{153}$ C. J. E. Suster, ${ }^{154}$ M. R. Sutton, ${ }^{153}$ S. Suzuki, ${ }^{81}$ M. Svatos,${ }^{136}$ M. Swiatlowski, ${ }^{36}$ S. P. Swift, ${ }^{2}$ A. Sydorenko, ${ }^{97}$ I. Sykora ${ }^{28 a}$ T. Sykora, ${ }^{138}$ D. Ta ${ }^{97}$ K. Tackmann, ${ }^{46}$ J. Taenzer, ${ }^{158}$ A. Taffard,${ }^{168}$ R. Tafirout, ${ }^{165 a}$ E. Tahirovic,${ }^{90}$ N. Taiblum, ${ }^{158} \mathrm{H}$. Takai, ${ }^{29}$ R. Takashima ${ }^{84}$ E. H. Takasugi, ${ }^{113}$ K. Takeda, ${ }^{82}$ T. Takeshita, ${ }^{147}$ Y. Takubo ${ }^{81}$ M. Talby, ${ }^{99}$ A. A. Talyshev, ${ }^{120 b, 120 a}$ J. Tanaka, ${ }^{160}$ M. Tanaka, ${ }^{162}$ R. Tanaka, ${ }^{128}$ R. Tanioka, ${ }^{82}$ B. B. Tannenwald, ${ }^{122}$ S. Tapia Araya, ${ }^{144 b}$ S. Tapprogge, ${ }^{97}$ A. Tarek Abouelfadl Mohamed, ${ }^{94}$ S. Tarem, ${ }^{157}$ G. Tarna, ${ }^{27 b, d}$ G. F. Tartarelli, ${ }^{69 a}$ P. Tas,${ }^{138}$ M. Tasevsky, ${ }^{136}$ T. Tashiro, ${ }^{83}$ E. Tassi ${ }^{40 b, 40 a}$ A. Tavares Delgado, ${ }^{135 a, 135 b}$ Y. Tayalati, ${ }^{34 \mathrm{e}}$ A. C. Taylor, ${ }^{116}$ A. J. Taylor, ${ }^{50}$ G. N. Taylor, ${ }^{102}$ P. T. E. Taylor, ${ }^{102}$ W. Taylor, ${ }^{165 b}$ A. S. Tee, ${ }^{87}$ P. Teixeira-Dias, ${ }^{91}$ H. Ten Kate, ${ }^{35}$ P. K. Teng, ${ }^{155}$ J. J. Teoh, ${ }^{129}$ F. Tepel, ${ }^{179}$ S. Terada, ${ }^{81}$ K. Terashi, ${ }^{160}$ J. Terron, ${ }^{96}$ S. Terzo, ${ }^{14}$ M. Testa, ${ }^{52}$ R. J. Teuscher, ${ }^{164, a g}$ S. J. Thais, ${ }^{180}$ T. Theveneaux-Pelzer, ${ }^{46}$ F. Thiele, ${ }^{39}$ J. P. Thomas, ${ }^{21}$ A. S. Thompson, ${ }^{58}$ P. D. Thompson, ${ }^{21}$ L. A. Thomsen, ${ }^{180}$ E. Thomson, ${ }^{132}$ Y. Tian, ${ }^{38}$ R. E. Ticse Torres, ${ }^{54}$ V. O. Tikhomirov, ${ }^{108, a 0}$ Yu. A. Tikhonov, ${ }^{120 b, 120 a}$ S. Timoshenko, ${ }^{110} \mathrm{P}$. Tipton, ${ }^{180} \mathrm{~S}$. Tisserant, ${ }^{99} \mathrm{~K}$. Todome, ${ }^{162}$ S. Todorova-Nova, ${ }^{5}$ S. Todt, ${ }^{48}$ J. Tojo, ${ }^{85}$ S. Tokár, ${ }^{28 a}$ K. Tokushuku, ${ }^{81}$ E. Tolley ${ }^{122}$ K. G. Tomiwa, ${ }^{32 c}$ M. Tomoto, ${ }^{115}$ L. Tompkins, ${ }^{150, p}$ K. Toms, ${ }^{116}$ B. Tong,${ }^{60}$ P. Tornambe, ${ }^{53}$ E. Torrence, ${ }^{127}$ H. Torres, ${ }^{48}$ E. Torró Pastor, ${ }^{145}$ C. Tosciri, ${ }^{131}$ J. Toth, ${ }^{99, \text { af }}$ F. Touchard, ${ }^{99}$ D. R. Tovey, ${ }^{146}$ C. J. Treado, ${ }^{121}$ T. Trefzger, ${ }^{174}$ F. Tresoldi, ${ }^{153}$ A. Tricoli, ${ }^{29}$ I. M. Trigger,${ }^{165 a}$ S. Trincaz-Duvoid, ${ }^{94}$ M. F. Tripiana, ${ }^{14}$ W. Trischuk, ${ }^{164}$ B. Trocmé,${ }^{59}$ A. Trofymov ${ }^{128}$ C. Troncon, ${ }^{69 a}$ M. Trovatelli, ${ }^{173}$ F. Trovato, ${ }^{153}$ L. Truong, ${ }^{32 b}$ M. Trzebinski, ${ }^{42}$ A. Trzupek, ${ }^{42}$ F. Tsai, ${ }^{46}$ J. C-L. Tseng, ${ }^{131}$ P. V. Tsiareshka, ${ }^{105}$ N. Tsirintanis, ${ }^{9}$ V. Tsiskaridze, ${ }^{152}$ E. G. Tskhadadze, ${ }^{156 a}$ I. I. Tsukerman, ${ }^{109}$ V. Tsulaia, ${ }^{18}$ S. Tsuno, ${ }^{81}$ D. Tsybychev, ${ }^{152}$ Y. Tu, ${ }^{64 b}$ A. Tudorache, ${ }^{27 \mathrm{~b}}$ V. Tudorache, ${ }^{27 \mathrm{~b}}$ T. T. Tulbure, ${ }^{27 \mathrm{a}}$ A. N. Tuna, ${ }^{60} \mathrm{~S}$. Turchikhin, ${ }^{80}$ D. Turgeman, ${ }^{177}$ I. Turk Cakir, ${ }^{4 b, w}$ R. Turra, ${ }^{69 \mathrm{a}}$ P. M. Tuts, ${ }^{38}$ E. Tzovara, ${ }^{97}$ G. Ucchielli, ${ }^{23 b, 23 \mathrm{a}}$ I. Ueda,${ }^{81}$ M. Ughetto, ${ }^{45 a, 45 b}$ F. Ukegawa, ${ }^{166}$ G. Unal, ${ }^{35}$ A. Undrus, ${ }^{29}$ G. Unel, ${ }^{168}$ F. C. Ungaro, ${ }^{102}$ Y. Unno, ${ }^{81}$ K. Uno, ${ }^{160}$ J. Urban,${ }^{28 b}$ P. Urquijo, ${ }^{102}$ P. Urrejola, ${ }^{97}$ G. Usai, ${ }^{8}$ J. Usui,${ }^{81}$ L. Vacavant, ${ }^{99}$ V. Vacek, ${ }^{137}$ B. Vachon, ${ }^{101}$ K. O. H. Vadla, ${ }^{130}$ A. Vaidya, ${ }^{92}$ C. Valderanis, ${ }^{112}$ E. Valdes Santurio, ${ }^{45 a, 45 b}$ M. Valente, ${ }^{55}$ S. Valentinetti, ${ }^{23 b, 23 a}$ A. Valero, ${ }^{171}$ L. Valéry, ${ }^{46}$ R. A. Vallance, ${ }^{21}$ A. Vallier, ${ }^{5}$ J. A. Valls Ferrer, ${ }^{171}$ T. R. Van Daalen, ${ }^{14}$ W. Van Den Wollenberg, ${ }^{118}$ H. van der Graaf, ${ }^{118}$ P. van Gemmeren, ${ }^{6}$ J. Van Nieuwkoop, ${ }^{149}$ I. van Vulpen, ${ }^{118}$ M. C. van Woerden, ${ }^{118}$ M. Vanadia, ${ }^{74 a, 74 b}$ W. Vandelli, ${ }^{35}$ A. Vaniachine, ${ }^{163}$ P. Vankov, ${ }^{118}$ R. Vari, ${ }^{73 a}$ E. W. Varnes, ${ }^{7}$ C. Varni, ${ }^{56,56 a}$ T. Varol, ${ }^{43}$ D. Varouchas, ${ }^{128}$ K. E. Varvell, ${ }^{154}$ G. A. Vasquez, ${ }^{144 b}$ J. G. Vasquez, ${ }^{180}$ F. Vazeille, ${ }^{37}$ D. Vazquez Furelos, ${ }^{14}$ T. Vazquez Schroeder, ${ }^{101}$ J. Veatch,${ }^{54}$ V. Vecchio, ${ }^{75 a}, 75 b$ L. M. Veloce, ${ }^{164}$ F. Veloso, ${ }^{135 a, 135 \mathrm{c}}$ S. Veneziano, ${ }^{73 \mathrm{a}}$ A. Ventura, ${ }^{68 \mathrm{a}, 68 \mathrm{~b}}$ M. Venturi, ${ }^{173} \mathrm{~N}$. Venturi, ${ }^{35}$ V. Vercesi, ${ }^{71 \mathrm{a}} \mathrm{M}$. Verducci, ${ }^{75 a, 75 b}$ 
C. M. Vergel Infante, ${ }^{79}$ W. Verkerke, ${ }^{118}$ A. T. Vermeulen, ${ }^{118}$ J. C. Vermeulen, ${ }^{118}$ M. C. Vetterli, ${ }^{149, \text { au }}$ N. Viaux Maira, ${ }^{144 b}$ O. Viazlo, ${ }^{95}$ I. Vichou, ${ }^{170, \dagger}$ T. Vickey, ${ }^{146}$ O. E. Vickey Boeriu, ${ }^{146}$ G. H. A. Viehhauser, ${ }^{131}$ S. Viel, ${ }^{18}$ L. Vigani, ${ }^{131}$ M. Villa, ${ }^{23 b, 23 a}$ M. Villaplana Perez,${ }^{69 a, 69 b}$ E. Vilucchi, ${ }^{52}$ M. G. Vincter, ${ }^{33}$ V. B. Vinogradov, ${ }^{80}$ A. Vishwakarma, ${ }^{46}$ C. Vittori, ${ }^{23 b, 23 a}$ I. Vivarelli, ${ }^{153}$ S. Vlachos,${ }^{10}$ M. Vogel, ${ }^{179}$ P. Vokac, ${ }^{137}$ G. Volpi,${ }^{14}$ S. E. von Buddenbrock,${ }^{32 c}$ E. von Toerne ${ }^{24}$ V. Vorobel ${ }^{138}$ K. Vorobev,${ }^{110}$ M. Vos,${ }^{171}$ J. H. Vossebeld, ${ }^{88}$ N. Vranjes,${ }^{16}$ M. Vranjes Milosavljevic, ${ }^{16}$ V. Vrba, ${ }^{137}$ M. Vreeswijk, ${ }^{118}$ R. Vuillermet, ${ }^{35}$ I. Vukotic, ${ }^{36}$ P. Wagner, ${ }^{24}$ W. Wagner, ${ }^{179}$ J. Wagner-Kuhr, ${ }^{112}$ H. Wahlberg, ${ }^{86}$ S. Wahrmund, ${ }^{48}$ K. Wakamiya, ${ }^{82}$ V. M. Walbrecht, ${ }^{113}$ J. Walder, ${ }^{87}$ R. Walker, ${ }^{112}$ W. Walkowiak, ${ }^{148}$ V. Wallangen, ${ }^{45 a, 45 b}$ A. M. Wang, ${ }^{60}$ C. Wang, ${ }^{61 b, d}$ F. Wang, ${ }^{178}$ H. Wang ${ }^{18}$ H. Wang, ${ }^{3}$ J. Wang, ${ }^{154}$ J. Wang, ${ }^{62 b}$ P. Wang, ${ }^{43}$ Q. Wang, ${ }^{124}$ R.-J. Wang, ${ }^{94}$ R. Wang, ${ }^{61 \mathrm{a}}$ R. Wang, ${ }^{6}$ S. M. Wang, ${ }^{155}$ W. Wang, ${ }^{155, \mathrm{n}}$ W. Wang, ${ }^{61 \mathrm{a}, \mathrm{ah}} \mathrm{W}$. Wang, ${ }^{61 \mathrm{a}}$ Y. Wang, ${ }^{61 \mathrm{a}} \mathrm{Z}$. Wang, ${ }^{61 \mathrm{c}}$ C. Wanotayaroj, ${ }^{46}$ A. Warburton, ${ }^{101}$ C. P. Ward, ${ }^{31}$ D. R. Wardrope, ${ }^{92}$ A. Washbrook,${ }^{50}$ P. M. Watkins, ${ }^{21}$ A. T. Watson, ${ }^{21}$ M. F. Watson, ${ }^{21}$ G. Watts, ${ }^{145}$ S. Watts, ${ }^{98}$ B. M. Waugh, ${ }^{92}$ A. F. Webb, ${ }^{11}$ S. Webb,${ }^{97}$ C. Weber, ${ }^{180}$ M. S. Weber, ${ }^{20}$ S. A. Weber, ${ }^{33}$ S. M. Weber, ${ }^{62 a}$ J. S. Webster, ${ }^{6}$ A. R. Weidberg, ${ }^{131}$ B. Weinert, ${ }^{66}$ J. Weingarten, ${ }^{54}$ M. Weirich, ${ }^{97}$ C. Weiser, ${ }^{53}$ P. S. Wells, ${ }^{35}$ T. Wenaus, ${ }^{29}$ T. Wengler, ${ }^{35}$ S. Wenig, ${ }^{35}$ N. Wermes, ${ }^{24}$ M. D. Werner, ${ }^{79}$ P. Werner, ${ }^{35}$ M. Wessels, ${ }^{62 a}$ T. D. Weston, ${ }^{20}$ K. Whalen, ${ }^{127}$ N. L. Whallon, ${ }^{145}$ A. M. Wharton, ${ }^{87}$ A. S. White, ${ }^{103}$ A. White, ${ }^{8}$ M. J. White, ${ }^{1}$ R. White, ${ }^{144 b}$ D. Whiteson, ${ }^{168}$ B. W. Whitmore ${ }^{87}$ F. J. Wickens, ${ }^{140}$ W. Wiedenmann, ${ }^{178}$ M. Wielers, ${ }^{140}$ C. Wiglesworth, ${ }^{39}$

L. A. M. Wiik-Fuchs, ${ }^{53}$ A. Wildauer, ${ }^{113}$ F. Wilk, ${ }^{98}$ H. G. Wilkens, ${ }^{35}$ L. J. Wilkins, ${ }^{91}$ H. H. Williams, ${ }^{132}$ S. Williams,${ }^{31}$

C. Willis, ${ }^{104}$ S. Willocq, ${ }^{100}$ J. A. Wilson, ${ }^{21}$ I. Wingerter-Seez, ${ }^{5}$ E. Winkels, ${ }^{153}$ F. Winklmeier, ${ }^{127}$ O. J. Winston, ${ }^{153}$

B. T. Winter, ${ }^{24}$ M. Wittgen, ${ }^{150}$ M. Wobisch, ${ }^{93, a l}$ A. Wolf,,${ }^{97}$ T. M. H. Wolf, ${ }^{118}$ R. Wolff, ${ }^{99}$ M. W. Wolter, ${ }^{42}$ H. Wolters, ${ }^{135 a, 135 c}$ V. W. S. Wong, ${ }^{172}$ N. L. Woods, ${ }^{143}$ S. D. Worm, ${ }^{21}$ B. K. Wosiek, ${ }^{42}$ K. W. Woźniak, ${ }^{42}$ K. Wraight, ${ }^{58}$ M. Wu, ${ }^{36}$ S. L. Wu, ${ }^{178}$ X. Wu, ${ }^{55}$ Y. Wu, ${ }^{61 a}$ T. R. Wyatt, ${ }^{98}$ B. M. Wynne, ${ }^{50}$ S. Xella, ${ }^{39}$ Z. Xi, ${ }^{103}$ L. Xia, ${ }^{175}$ D. Xu, ${ }^{15 a}$ H. Xu, ${ }^{61 a}$ L. Xu, ${ }^{29}$ T. Xu, ${ }^{142}$ W. Xu, ${ }^{103}$ B. Yabsley, ${ }^{154}$ S. Yacoob,${ }^{32 a}$ K. Yajima, ${ }^{129}$ D. P. Yallup,${ }^{92}$ D. Yamaguchi,${ }^{162}$ Y. Yamaguchi, ${ }^{162}$ A. Yamamoto, ${ }^{81}$

T. Yamanaka, ${ }^{160}$ F. Yamane, ${ }^{82}$ M. Yamatani, ${ }^{160}$ T. Yamazaki, ${ }^{160}$ Y. Yamazaki, ${ }^{82}$ Z. Yan, ${ }^{25}$ H. Yang, ${ }^{61 c, 61 d}$ H. Yang, ${ }^{18}$ S. Yang, ${ }^{78}$ Y. Yang, ${ }^{160}$ Z. Yang, ${ }^{17}$ W-M. Yao, ${ }^{18}$ Y. C. Yap,${ }^{46}$ Y. Yasu, ${ }^{81}$ E. Yatsenko, ${ }^{61 c, 61 d}$ J. Ye, ${ }^{43}$ S. Ye ${ }^{29}$ I. Yeletskikh,${ }^{80}$ E. Yigitbasi, ${ }^{25}$ E. Yildirim, ${ }^{97}$ K. Yorita,${ }^{176}$ K. Yoshihara, ${ }^{132}$ C. J. S. Young, ${ }^{35}$ C. Young, ${ }^{150}$ J. Yu, ${ }^{8}$ J. Yu,${ }^{79}$ X. Yue, ${ }^{62 a}$ S. P. Y. Yuen, ${ }^{24}$ I. Yusuff,,${ }^{31, a w}$ B. Zabinski, ${ }^{42}$ G. Zacharis, ${ }^{10}$ E. Zaffaroni, ${ }^{55}$ R. Zaidan, ${ }^{14}$ A. M. Zaitsev, ${ }^{139, \text { an }}$ N. Zakharchuk, ${ }^{46}$ J. Zalieckas ${ }^{17}$ S. Zambito, ${ }^{60}$ D. Zanzi, ${ }^{35}$ D. R. Zaripovas, ${ }^{58}$ S. V. Zeißner, ${ }^{47}$ C. Zeitnitz, ${ }^{179}$ G. Zemaityte, ${ }^{131}$ J. C. Zeng, ${ }^{170}$ Q. Zeng, ${ }^{150}$ O. Zenin, ${ }^{139}$ T. Ženiš, ${ }^{28 a}$ D. Zerwas, ${ }^{128}$ M. Zgubič ${ }^{131}$ D. Zhang, ${ }^{103}$ D. Zhang, ${ }^{61 b}$ F. Zhang, ${ }^{178}$ G. Zhang, ${ }^{61 \text { a,ah }}$ H. Zhang, ${ }^{15 b}$ J. Zhang, ${ }^{6}$ L. Zhang, ${ }^{53}$ L. Zhang, ${ }^{61 \mathrm{a}}$ M. Zhang, ${ }^{170}$ P. Zhang, ${ }^{15 \mathrm{~b}}$ R. Zhang, ${ }^{61 \mathrm{a}, \mathrm{d}}$ R. Zhang, ${ }^{24}$ X. Zhang, ${ }^{61 \mathrm{~b}}$ Y. Zhang, ${ }^{15 \mathrm{~d}}$ Z. Zhang, ${ }^{128}$ X. Zhao, ${ }^{43}$ Y. Zhao, ${ }^{61 \mathrm{~b}, \mathrm{ak}}$ Z. Zhao, ${ }^{61 \mathrm{a}}$ A. Zhemchugov ${ }^{80}$ B. Zhou, ${ }^{103}$ C. Zhou, ${ }^{178}$ L. Zhou, ${ }^{43}$ M. Zhou, ${ }^{15 \mathrm{~d}}$ M. Zhou, ${ }^{152}$ N. Zhou, ${ }^{61 \mathrm{c}}$ Y. Zhou, ${ }^{7}$ C. G. Zhu, ${ }^{61 \mathrm{~b}}$ H. Zhu, ${ }^{61 \mathrm{a}}$ H. Zhu, ${ }^{15 \mathrm{a}}$ J. Zhu, ${ }^{103}$ Y. Zhu, ${ }^{61 \mathrm{a}}$ X. Zhuang, ${ }^{15 a}$ K. Zhukov, ${ }^{108}$ V. Zhulanov, ${ }^{120 b, 120 a}$ A. Zibell, ${ }^{174}$ D. Zieminska, ${ }^{66}$ N. I. Zimine, ${ }^{80}$ S. Zimmermann, ${ }^{53}$ Z. Zinonos, ${ }^{113}$ M. Zinser, ${ }^{97}$ M. Ziolkowski, ${ }^{148}$ L. Živkovici, ${ }^{16}$ G. Zobernig, ${ }^{178}$ A. Zoccoli, ${ }^{23 b, 23 a}$ K. Zoch, ${ }^{54}$ T. G. Zorbas, ${ }^{146}$ R. Zou, ${ }^{36}$ M. zur Nedden, ${ }^{19}$ and L. Zwalinski ${ }^{35}$

(ATLAS Collaboration)

${ }^{1}$ Department of Physics, University of Adelaide, Adelaide, Australia ${ }^{2}$ Physics Department, SUNY Albany, Albany, New York, USA

${ }^{3}$ Department of Physics, University of Alberta, Edmonton, Alberta, Canada

${ }^{4 a}$ Department of Physics, Ankara University, Ankara

${ }^{4 \mathrm{~b}}$ Istanbul Aydin University, Istanbul

${ }^{4 \mathrm{c}}$ Division of Physics, TOBB University of Economics and Technology, Ankara, Turkey

${ }^{5}$ LAPP, Université Grenoble Alpes, Université Savoie Mont Blanc, CNRS/IN2P3, Annecy, France

${ }^{6}$ High Energy Physics Division, Argonne National Laboratory, Argonne, Illinois, USA

${ }^{7}$ Department of Physics, University of Arizona, Tucson, Arizona, USA

${ }^{8}$ Department of Physics, The University of Texas at Arlington, Arlington, Texas, USA

${ }^{9}$ Physics Department, National and Kapodistrian University of Athens, Athens, Greece

${ }^{10}$ Physics Department, National Technical University of Athens, Zografou, Greece

${ }^{11}$ Department of Physics, The University of Texas at Austin, Austin, Texas, USA

${ }^{12 a}$ Bahcesehir University, Faculty of Engineering and Natural Sciences, Istanbul

${ }^{12 \mathrm{~b}}$ Istanbul Bilgi University, Faculty of Engineering and Natural Sciences, Istanbul

${ }^{12 c}$ Department of Physics, Bogazici University, Istanbul 
${ }^{12 \mathrm{~d}}$ Department of Physics Engineering, Gaziantep University, Gaziantep, Turkey

${ }^{13}$ Institute of Physics, Azerbaijan Academy of Sciences, Baku, Azerbaijan

${ }^{14}$ Institut de Física d'Altes Energies (IFAE), The Barcelona Institute of Science and Technology, Barcelona, Spain

${ }^{15 a}$ Institute of High Energy Physics, Chinese Academy of Sciences, Beijing

${ }^{15 \mathrm{~b}}$ Department of Physics, Nanjing University, Jiangsu

${ }^{15 c}$ Physics Department, Tsinghua University, Beijing

${ }^{15 \mathrm{~d}}$ University of Chinese Academy of Science (UCAS), Beijing, China

${ }^{16}$ Institute of Physics, University of Belgrade, Belgrade, Serbia

${ }^{17}$ Department for Physics and Technology, University of Bergen, Bergen, Norway

${ }^{18}$ Physics Division, Lawrence Berkeley National Laboratory and University of California, Berkeley, California, USA

${ }^{19}$ Department of Physics, Humboldt University, Berlin, Germany

${ }^{20}$ Albert Einstein Center for Fundamental Physics and Laboratory for High Energy Physics, University of Bern, Bern, Switzerland

${ }^{21}$ School of Physics and Astronomy, University of Birmingham, Birmingham, United Kingdom

${ }^{22}$ Centro de Investigaciones, Universidad Antonio Narino, Bogota, Colombia

${ }^{23 a}$ Dipartimento di Fisica e Astronomia, Università di Bologna, Bologna

${ }^{23 \mathrm{~b}}$ INFN Sezione di Bologna, Italy

${ }^{24}$ Physikalisches Institut, University of Bonn, Bonn, Germany

${ }^{25}$ Department of Physics, Boston University, Boston, Massachusetts, USA

${ }^{26}$ Department of Physics, Brandeis University, Waltham, Massachusetts, USA

${ }^{27}$ Transilvania University of Brasov, Brasov

${ }^{27 \mathrm{~b}}$ Horia Hulubei National Institute of Physics and Nuclear Engineering

${ }^{27 \mathrm{c}}$ Department of Physics, Alexandru Ioan Cuza University of Iasi, Iasi

${ }^{27 \mathrm{~d}}$ National Institute for Research and Development of Isotopic and Molecular Technologies, Physics Department, Cluj Napoca

${ }^{27 \mathrm{e}}$ University Politehnica Bucharest, Bucharest

${ }^{27 \mathrm{f}}$ West University in Timisoara, Timisoara, Romania

${ }^{28 \mathrm{a}}$ Faculty of Mathematics, Physics and Informatics, Comenius University, Bratislava

${ }^{28 \mathrm{~b}}$ Department of Subnuclear Physics, Institute of Experimental Physics of the Slovak Academy of Sciences, Kosice, Slovak Republic

${ }^{29}$ Physics Department, Brookhaven National Laboratory, Upton, New York, USA

${ }^{30}$ Departamento de Física, Universidad de Buenos Aires, Buenos Aires, Argentina

${ }^{31}$ Cavendish Laboratory, University of Cambridge, Cambridge, United Kingdom

${ }^{32 a}$ Department of Physics, University of Cape Town, Cape Town, South Africa

${ }^{32 \mathrm{~b}}$ Department of Mechanical Engineering Science, University of Johannesburg, Johannesburg, South Africa

${ }^{32 c}$ School of Physics, University of the Witwatersrand, Johannesburg, South Africa

${ }^{33}$ Department of Physics, Carleton University, Ottawa, Ontario, Canada

${ }^{34 a}$ Faculté des Sciences Ain Chock, Réseau Universitaire de Physique des Hautes Energies - Université Hassan II, Casablanca

${ }^{34 \mathrm{~b}}$ Centre National de l'Energie des Sciences Techniques Nucleaires, Rabat

${ }^{34 \mathrm{c}}$ Faculté des Sciences Semlalia, Université Cadi Ayyad, LPHEA-Marrakech

${ }^{34 \mathrm{~d}}$ Faculté des Sciences, Université Mohamed Premier and LPTPM, Oujda

${ }^{34 \mathrm{e}}$ Faculté des sciences, Université Mohammed V, Rabat, Morocco

${ }^{35}$ CERN, Geneva, Switzerland

${ }^{36}$ Enrico Fermi Institute, University of Chicago, Chicago, Illinois, USA

${ }^{37}$ LPC, Université Clermont Auvergne, CNRS/IN2P3, Clermont-Ferrand, France

${ }^{38}$ Nevis Laboratory, Columbia University, Irvington, New York, USA

${ }^{39}$ Niels Bohr Institute, University of Copenhagen, Kobenhavn, Denmark

${ }^{40 a}$ Dipartimento di Fisica, Università della Calabria, Rende

${ }^{40 \mathrm{~b}}$ INFN Gruppo Collegato di Cosenza, Laboratori Nazionali di Frascati, Italy

${ }^{41 \mathrm{a}}$ AGH University of Science and Technology, Faculty of Physics and Applied Computer Science, Krakow

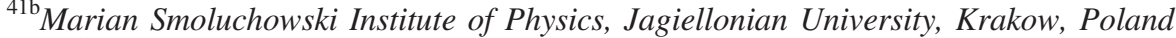

${ }^{42}$ Institute of Nuclear Physics Polish Academy of Sciences, Krakow, Poland

${ }^{43}$ Physics Department, Southern Methodist University, Dallas, Texas, USA

${ }^{44}$ Physics Department, University of Texas at Dallas, Richardson, Texas, USA 
${ }^{45 a}$ Department of Physics, Stockholm University

${ }^{45 \mathrm{~b}}$ The Oskar Klein Centre, Stockholm, Sweden

${ }^{46}$ DESY, Hamburg and Zeuthen, Germany

${ }^{47}$ Lehrstuhl für Experimentelle Physik IV, Technische Universität Dortmund, Dortmund, Germany

${ }^{48}$ Institut für Kern- und Teilchenphysik, Technische Universität Dresden, Dresden, Germany

${ }^{49}$ Department of Physics, Duke University, Durham, North Carolina, USA

${ }^{50}$ SUPA - School of Physics and Astronomy, University of Edinburgh, Edinburgh, United Kingdom

${ }^{51}$ Centre de Calcul de l'Institut National de Physique Nucléaire et de Physique des Particules (IN2P3), Villeurbanne, France

${ }^{52}$ INFN e Laboratori Nazionali di Frascati, Frascati, Italy

${ }^{53}$ Fakultät für Mathematik und Physik, Albert-Ludwigs-Universität, Freiburg, Germany

${ }^{54}$ II Physikalisches Institut, Georg-August-Universität, Göttingen, Germany

${ }^{55}$ Departement de Physique Nucléaire et Corpusculaire, Université de Genève, Geneva, Switzerland

${ }^{56 a}$ Dipartimento di Fisica, Università di Genova, Genova ${ }^{56 \mathrm{~b}}$ INFN Sezione di Genova, Italy

${ }^{57}$ II. Physikalisches Institut, Justus-Liebig-Universität Giessen, Giessen, Germany

${ }^{58}$ SUPA - School of Physics and Astronomy, University of Glasgow, Glasgow, United Kingdom

${ }^{59}$ LPSC, Université Grenoble Alpes, CNRS/IN2P3, Grenoble INP, Grenoble, France

${ }^{60}$ Laboratory for Particle Physics and Cosmology, Harvard University, Cambridge, Massachusetts, USA

${ }^{61 \mathrm{a}}$ Department of Modern Physics and State Key Laboratory of Particle Detection and Electronics,

University of Science and Technology of China, Anhui

${ }^{61 \mathrm{~b}}$ School of Physics, Shandong University, Shandong

${ }^{61 \mathrm{c} S c h o o l}$ of Physics and Astronomy, Key Laboratory for Particle Physics, Astrophysics and Cosmology,

Ministry of Education, Shanghai Key Laboratory for Particle Physics and Cosmology,

Shanghai Jiao Tong University

${ }^{61 \mathrm{~d}}$ Tsung-Dao Lee Institute, Shanghai, China

${ }^{62 a}$ Kirchhoff-Institut für Physik, Ruprecht-Karls-Universität Heidelberg, Heidelberg

${ }^{62 \mathrm{~b}}$ Physikalisches Institut, Ruprecht-Karls-Universität Heidelberg, Heidelberg, Germany

${ }^{63}$ Faculty of Applied Information Science, Hiroshima Institute of Technology, Hiroshima, Japan

${ }^{64 a}$ Department of Physics, The Chinese University of Hong Kong, Shatin, N.T., Hong Kong

${ }^{64 \mathrm{~b}}$ Department of Physics, The University of Hong Kong, Hong Kong

${ }^{64 \mathrm{c}}$ Department of Physics and Institute for Advanced Study, The Hong Kong University of Science and

Technology, Clear Water Bay, Kowloon, Hong Kong, China

${ }^{65}$ Department of Physics, National Tsing Hua University, Hsinchu, Taiwan

${ }^{66}$ Department of Physics, Indiana University, Bloomington, Indiana, USA

${ }^{67 \mathrm{a}}$ INFN Gruppo Collegato di Udine, Sezione di Trieste, Udine

${ }^{67 \mathrm{~b}}$ ICTP, Trieste

${ }^{67 \mathrm{c}}$ Dipartimento di Chimica, Fisica e Ambiente, Università di Udine, Udine, Italy

${ }^{68 \mathrm{a}}$ INFN Sezione di Lecce, Lecce, Italy

${ }^{68 \mathrm{~b}}$ Dipartimento di Matematica e Fisica, Università del Salento, Lecce, Italy

${ }^{69 a}$ INFN Sezione di Milano, Milano, Italy

${ }^{69 \mathrm{~b}}$ Dipartimento di Fisica, Università di Milano, Milano, Italy

${ }^{70 a}$ INFN Sezione di Napoli, Napoli, Italy

${ }^{70 \mathrm{~b}}$ Dipartimento di Fisica, Università di Napoli, Napoli, Italy

${ }^{71 \mathrm{a}}$ INFN Sezione di Pavia, Pavia, Italy

${ }^{71 \mathrm{~b}}$ Dipartimento di Fisica, Università di Pavia, Pavia, Italy

${ }^{72 a}$ INFN Sezione di Pisa, Pisa, Italy

${ }^{72 b}$ Dipartimento di Fisica E. Fermi, Università di Pisa, Pisa, Italy

${ }^{73}$ INFN Sezione di Roma, Roma, Italy

${ }^{73 b}$ Dipartimento di Fisica, Sapienza Università di Roma, Roma, Italy

${ }^{74 a}$ INFN Sezione di Roma Tor Vergata, Roma, Italy

${ }^{74 \mathrm{~b}}$ Dipartimento di Fisica, Università di Roma Tor Vergata, Roma, Italy

${ }^{75 a}$ INFN Sezione di Roma Tre, Roma, Italy

${ }^{75 b}$ Dipartimento di Matematica e Fisica, Università Roma Tre, Roma, Italy

${ }^{76 a}$ INFN-TIFPA, Italy

${ }^{76 \mathrm{~b}}$ University of Trento, Trento, Italy

${ }^{77}$ Institut für Astro- und Teilchenphysik, Leopold-Franzens-Universität, Innsbruck, Austria

${ }^{78}$ University of Iowa, Iowa City, Iowa, USA 
${ }^{79}$ Department of Physics and Astronomy, Iowa State University, Ames, Iowa, USA

${ }^{80}$ Joint Institute for Nuclear Research, JINR Dubna, Dubna, Russia

${ }^{81}$ KEK, High Energy Accelerator Research Organization, Tsukuba, Japan

${ }^{82}$ Graduate School of Science, Kobe University, Kobe, Japan

${ }^{83}$ Faculty of Science, Kyoto University, Kyoto, Japan

${ }^{84}$ Kyoto University of Education, Kyoto, Japan

${ }^{85}$ Research Center for Advanced Particle Physics and Department of Physics, Kyushu University, Fukuoka, Japan

${ }^{86}$ Instituto de Física La Plata, Universidad Nacional de La Plata and CONICET, La Plata, Argentina

${ }^{87}$ Physics Department, Lancaster University, Lancaster, United Kingdom

${ }^{88}$ Oliver Lodge Laboratory, University of Liverpool, Liverpool, United Kingdom

${ }^{89}$ Department of Experimental Particle Physics, Jožef Stefan Institute and Department of Physics, University of Ljubljana, Ljubljana, Slovenia

${ }^{90}$ School of Physics and Astronomy, Queen Mary University of London, London, United Kingdom

${ }^{91}$ Department of Physics, Royal Holloway University of London, Surrey, United Kingdom

${ }^{92}$ Department of Physics and Astronomy, University College London, London, United Kingdom

${ }^{93}$ Louisiana Tech University, Ruston, Louisiana, USA

${ }^{94}$ Laboratoire de Physique Nucléaire et de Hautes Energies, UPMC and Université Paris-Diderot and CNRS/IN2P3, Paris, France

${ }^{95}$ Fysiska institutionen, Lunds universitet, Lund, Sweden

${ }^{96}$ Departamento de Fisica Teorica C-15 and CIAFF, Universidad Autonoma de Madrid, Madrid, Spain

${ }^{97}$ Institut für Physik, Universität Mainz, Mainz, Germany

${ }^{98}$ School of Physics and Astronomy, University of Manchester, Manchester, United Kingdom

${ }^{99}$ CPPM, Aix-Marseille Université and CNRS/IN2P3, Marseille, France

${ }^{100}$ Department of Physics, University of Massachusetts, Amherst, Massachusetts, USA

${ }^{101}$ Department of Physics, McGill University, Montreal, Quebec, Canada

${ }^{102}$ School of Physics, University of Melbourne, Victoria, Australia

${ }^{103}$ Department of Physics, The University of Michigan, Ann Arbor, Michigan, USA

${ }^{104}$ Department of Physics and Astronomy, Michigan State University, East Lansing, Michigan, USA

${ }^{105}$ B.I. Stepanov Institute of Physics, National Academy of Sciences of Belarus, Minsk, Republic of Belarus

${ }^{106}$ Research Institute for Nuclear Problems of Byelorussian State University, Minsk, Republic of Belarus

${ }^{107}$ Group of Particle Physics, University of Montreal, Montreal, Quebec, Canada

${ }^{108}$ P.N. Lebedev Physical Institute of the Russian Academy of Sciences, Moscow, Russia

${ }^{109}$ Institute for Theoretical and Experimental Physics (ITEP), Moscow, Russia

${ }^{110}$ National Research Nuclear University MEPhI, Moscow, Russia

${ }^{111}$ D.V. Skobeltsyn Institute of Nuclear Physics, M.V. Lomonosov Moscow State University, Moscow, Russia

${ }^{112}$ Fakultät für Physik, Ludwig-Maximilians-Universität München, München, Germany

${ }^{113}$ Max-Planck-Institut für Physik (Werner-Heisenberg-Institut), München, Germany

${ }^{114}$ Nagasaki Institute of Applied Science, Nagasaki, Japan

${ }^{115}$ Graduate School of Science and Kobayashi-Maskawa Institute, Nagoya University, Nagoya, Japan

${ }^{116}$ Department of Physics and Astronomy, University of New Mexico, Albuquerque, New Mexico, USA

${ }^{117}$ Institute for Mathematics, Astrophysics and Particle Physics, Radboud University Nijmegen/Nikhef, Nijmegen, Netherlands

${ }^{118}$ Nikhef National Institute for Subatomic Physics and University of Amsterdam, Amsterdam, Netherlands

${ }^{119}$ Department of Physics, Northern Illinois University, DeKalb, Illinois, USA

${ }^{120 \mathrm{a}}$ Budker Institute of Nuclear Physics, SB RAS, Novosibirsk

${ }^{120 \mathrm{~b}}$ Novosibirsk State University Novosibirsk, Russia

${ }^{121}$ Department of Physics, New York University, New York, New York, USA

${ }^{122}$ The Ohio State University, Columbus, Ohio, USA

${ }^{123}$ Faculty of Science, Okayama University, Okayama, Japan

${ }^{124}$ Homer L. Dodge Department of Physics and Astronomy, University of Oklahoma, Norman, Oklahoma, USA

${ }^{125}$ Department of Physics, Oklahoma State University, Stillwater, Oklahoma, USA

${ }^{126}$ Palacký University, RCPTM, Olomouc, Czech Republic

${ }^{127}$ Center for High Energy Physics, University of Oregon, Eugene, Oregon, USA

${ }^{128}$ LAL, Université Paris-Sud, CNRS/IN2P3, Université Paris-Saclay, Orsay, France

${ }^{129}$ Graduate School of Science, Osaka University, Osaka, Japan 
${ }^{130}$ Department of Physics, University of Oslo, Oslo, Norway

${ }^{131}$ Department of Physics, Oxford University, Oxford, United Kingdom

${ }^{132}$ Department of Physics, University of Pennsylvania, Philadelphia, Pennsylvania, USA

${ }^{133}$ Konstantinov Nuclear Physics Institute of National Research Centre "Kurchatov Institute”, PNPI, St. Petersburg, Russia

${ }^{134}$ Department of Physics and Astronomy, University of Pittsburgh, Pittsburgh, Pennsylvania, USA

${ }^{135 a}$ Laboratório de Instrumentação e Física Experimental de Partículas - LIP, Lisboa

${ }^{135 \mathrm{~b}}$ Faculdade de Ciências, Universidade de Lisboa, Lisboa

${ }^{135 \mathrm{c}}$ Department of Physics, University of Coimbra, Coimbra

${ }^{135 \mathrm{~d}}$ Centro de Física Nuclear da Universidade de Lisboa, Lisboa

${ }^{135 \mathrm{e}}$ Departamento de Fisica, Universidade do Minho, Braga

${ }^{135 \mathrm{f}}$ Departamento de Fisica Teorica y del Cosmos, Universidad de Granada, Granada (Spain)

${ }^{135 \mathrm{~g}}$ Dep Fisica and CEFITEC of Faculdade de Ciencias e Tecnologia, Universidade Nova de Lisboa, Caparica, Portugal

${ }^{136}$ Institute of Physics, Academy of Sciences of the Czech Republic, Praha, Czech Republic

${ }^{137}$ Czech Technical University in Prague, Praha, Czech Republic

${ }^{138}$ Charles University, Faculty of Mathematics and Physics, Prague, Czech Republic

${ }^{139}$ State Research Center Institute for High Energy Physics (Protvino), NRC KI, Russia

${ }^{140}$ Particle Physics Department, Rutherford Appleton Laboratory, Didcot, United Kingdom

${ }^{141 a}$ Universidade Federal do Rio De Janeiro COPPE/EE/IF, Rio de Janeiro

${ }^{141 \mathrm{~b}}$ Electrical Circuits Department, Federal University of Juiz de Fora (UFJF), Juiz de Fora

${ }^{141 \mathrm{c}}$ Federal University of Sao Joao del Rei (UFSJ), Sao Joao del Rei

${ }^{141 \mathrm{~d} I n s t i t u t o}$ de Fisica, Universidade de Sao Paulo, Sao Paulo, Brazil

${ }^{142}$ Institut de Recherches sur les Lois Fondamentales de l'Univers, DSM/IRFU, CEA Saclay, Gif-sur-Yvette, France

${ }^{143}$ Santa Cruz, Institute for Particle Physics, University of California Santa Cruz, Santa Cruz, California, USA

${ }^{144 a}$ Departamento de Física, Pontificia Universidad Católica de Chile, Santiago

${ }^{144 b}$ Departamento de Física, Universidad Técnica Federico Santa María, Valparaíso, Chile

${ }^{145}$ Department of Physics, University of Washington, Seattle, Washington, USA

${ }^{146}$ Department of Physics and Astronomy, University of Sheffield, Sheffield, United Kingdom

${ }^{147}$ Department of Physics, Shinshu University, Nagano, Japan

${ }^{148}$ Department Physik, Universität Siegen, Siegen, Germany

${ }^{149}$ Department of Physics, Simon Fraser University, Burnaby, British Columbia, Canada

${ }^{150}$ SLAC National Accelerator Laboratory, Stanford, California, USA

${ }^{151}$ Physics Department, Royal Institute of Technology, Stockholm, Sweden

${ }^{152}$ Departments of Physics and Astronomy, Stony Brook University, Stony Brook, New York, USA

${ }^{153}$ Department of Physics and Astronomy, University of Sussex, Brighton, United Kingdom

${ }^{154}$ School of Physics, University of Sydney, Sydney, Australia

${ }^{155}$ Institute of Physics, Academia Sinica, Taipei, Taiwan

${ }^{156 a}$ E. Andronikashvili Institute of Physics, Iv. Javakhishvili Tbilisi State University, Tbilisi

${ }^{156 b}$ High Energy Physics Institute, Tbilisi State University, Tbilisi, Georgia

${ }^{157}$ Department of Physics, Technion: Israel Institute of Technology, Haifa, Israel

${ }^{158}$ Raymond and Beverly Sackler School of Physics and Astronomy, Tel Aviv University, Tel Aviv, Israel

${ }^{159}$ Department of Physics, Aristotle University of Thessaloniki, Thessaloniki, Greece

${ }^{160}$ International Center for Elementary Particle Physics and Department of Physics, The University of Tokyo, Tokyo, Japan

${ }^{161}$ Graduate School of Science and Technology, Tokyo Metropolitan University, Tokyo, Japan

${ }^{162}$ Department of Physics, Tokyo Institute of Technology, Tokyo, Japan

${ }^{163}$ Tomsk State University, Tomsk, Russia

${ }^{164}$ Department of Physics, University of Toronto, Toronto, Ontario, Canada

${ }^{165 a}$ TRIUMF, Vancouver, British Columbia, Canada

${ }^{165 \mathrm{~b}}$ Department of Physics and Astronomy, York University, Toronto, Ontario, Canada

${ }^{166}$ Division of Physics and Tomonaga Center for the History of the Universe, Faculty of Pure and Applied Sciences, University of Tsukuba, Tsukuba, Japan

${ }^{167}$ Department of Physics and Astronomy, Tufts University, Medford, Massachusetts, USA

${ }^{168}$ Department of Physics and Astronomy, University of California Irvine, Irvine, California, USA

${ }^{169}$ Department of Physics and Astronomy, University of Uppsala, Uppsala, Sweden 
${ }^{170}$ Department of Physics, University of Illinois, Urbana, Illinois, USA

${ }^{171}$ Instituto de Fisica Corpuscular (IFIC), Centro Mixto Universidad de Valencia - CSIC, Spain

${ }^{172}$ Department of Physics, University of British Columbia, Vancouver, British Columbia, Canada

${ }^{173}$ Department of Physics and Astronomy, University of Victoria, Victoria, British Columbia, Canada

${ }^{174}$ Fakultät für Physik und Astronomie, Julius-Maximilians-Universität, Würzburg, Germany

${ }^{175}$ Department of Physics, University of Warwick, Coventry, United Kingdom

${ }^{176}$ Waseda University, Tokyo, Japan

${ }^{177}$ Department of Particle Physics, The Weizmann Institute of Science, Rehovot, Israel

${ }^{178}$ Department of Physics, University of Wisconsin, Madison, Wisconsin, USA

${ }^{179}$ Fakultät für Mathematik und Naturwissenschaften, Fachgruppe Physik, Bergische Universität Wuppertal, Wuppertal, Germany

${ }^{180}$ Department of Physics, Yale University, New Haven, Connecticut, USA

${ }^{181}$ Yerevan Physics Institute, Yerevan, Armenia

${ }^{\dagger}$ Deceased.

${ }^{a}$ Also at Borough of Manhattan Community College, City University of New York, New York City, New York, USA.

${ }^{b}$ Also at Centre for High Performance Computing, CSIR Campus, Rosebank, Cape Town, South Africa.

${ }^{\mathrm{c}}$ Also at CERN, Geneva, Switzerland.

${ }^{\mathrm{d}}$ Also at CPPM, Aix-Marseille Université and CNRS/IN2P3, Marseille, France.

${ }^{\mathrm{e}}$ Also at Departament de Fisica de la Universitat Autonoma de Barcelona, Barcelona, Spain.

${ }_{\mathrm{f}}^{\mathrm{f}}$ Also at Departamento de Fisica Teorica y del Cosmos, Universidad de Granada, Granada (Spain), Spain.

${ }^{g}$ Also at Departement de Physique Nucléaire et Corpusculaire, Université de Genève, Geneva, Switzerland.

${ }^{\mathrm{h}}$ Also at Department of Financial and Management Engineering, University of the Aegean, Chios, Greece.

${ }^{i}$ Also at Department of Physics and Astronomy, University of Louisville, Louisville, Kentucky, USA.

${ }^{\mathrm{j}}$ Also at Department of Physics and Astronomy, University of Sheffield, Sheffield, United Kingdom.

${ }^{\mathrm{k}}$ Also at Department of Physics, California State University, Fresno, California, USA.

${ }^{1}$ Also at Department of Physics, California State University, Sacramento, California, USA.

${ }^{\mathrm{m}}$ Also at Department of Physics, King's College London, London, United Kingdom.

${ }^{\mathrm{n}}$ Also at Department of Physics, Nanjing University, Jiangsu, China.

${ }^{\circ}$ Also at Department of Physics, St. Petersburg State Polytechnical University, St. Petersburg, Russia.

${ }^{\mathrm{p}}$ Also at Department of Physics, Stanford University, Stanford, California, USA.

${ }^{\mathrm{q}}$ Also at Department of Physics, The University of Michigan, Ann Arbor, Michigan, USA.

${ }^{\mathrm{r}}$ Also at Department of Physics, University of Fribourg, Fribourg, Switzerland.

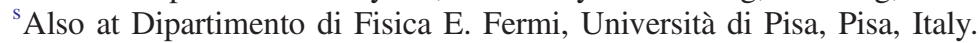

${ }^{\mathrm{t}}$ Also at Faculty of Physics, M.V.Lomonosov Moscow State University, Moscow, Russia.

${ }^{\mathrm{u}}$ Also at Fakultät für Mathematik und Physik, Albert-Ludwigs-Universität, Freiburg, Germany.

${ }^{v}$ Also at Georgian Technical University (GTU),Tbilisi, Georgia.

${ }^{\mathrm{w}}$ Also at Giresun University, Faculty of Engineering, Turkey.

${ }^{\mathrm{x}}$ Also at Graduate School of Science, Osaka University, Osaka, Japan.

${ }^{\mathrm{y}}$ Also at Hellenic Open University, Patras, Greece.

${ }^{\mathrm{z}}$ Also at Horia Hulubei National Institute of Physics and Nuclear Engineering, Romania.

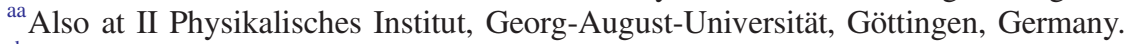

${ }^{\mathrm{ab}}$ Also at Institucio Catalana de Recerca i Estudis Avancats, ICREA, Barcelona, Spain.

${ }^{a c}$ Also at Institut de Física d'Altes Energies (IFAE), The Barcelona Institute of Science and Technology, Barcelona, Spain.

${ }^{\mathrm{ad}}$ Also at Institute for Mathematics, Astrophysics and Particle Physics, Radboud University Nijmegen/Nikhef, Nijmegen, Netherlands.

${ }^{\mathrm{ae}}$ Also at Institute for Nuclear Research and Nuclear Energy (INRNE) of the Bulgarian Academy of Sciences, Sofia, Bulgaria.

${ }^{a f}$ Also at Institute for Particle and Nuclear Physics, Wigner Research Centre for Physics, Budapest, Hungary.

${ }^{\mathrm{ag}}$ Also at Institute of Particle Physics (IPP), Canada.

${ }^{\text {ah }}$ Also at Institute of Physics, Academia Sinica, Taipei, Taiwan.

${ }^{\text {ai }}$ Also at Institute of Physics, Azerbaijan Academy of Sciences, Baku, Azerbaijan.

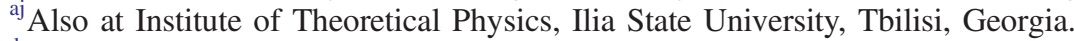

${ }^{\mathrm{ak}}$ Also at LAL, Université Paris-Sud, CNRS/IN2P3, Université Paris-Saclay, Orsay, France.

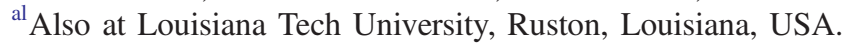

${ }^{a m}$ Also at Manhattan College, New York, New York, USA.

${ }^{\text {an }}$ Also at Moscow Institute of Physics and Technology State University, Dolgoprudny, Russia.

${ }^{\text {ao }}$ Also at National Research Nuclear University MEPhI, Moscow, Russia.

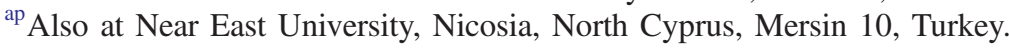

${ }^{\mathrm{aq}}$ Also at School of Physics, Sun Yat-sen University, Guangzhou, China.

${ }^{\mathrm{ar}}$ Also at The City College of New York, New York, New York, USA. 


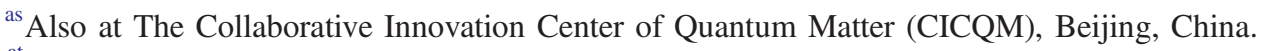

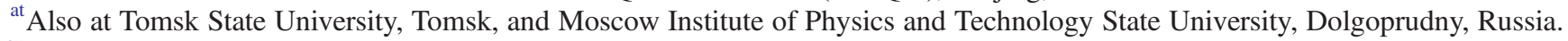

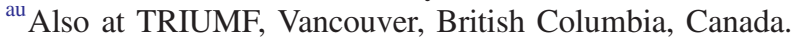

${ }^{a v}$ Also at Universita di Napoli Parthenope, Napoli, Italy.

${ }^{\text {aw }}$ Also at University of Malaya, Department of Physics, Kuala Lumpur, Malaysia. 